\title{
The mechanisms of serotonergic signaling in Drosophila
}

\author{
Xenia Borue \\ Princeton, NJ
}

B. A. Chemistry and Biology, Cornell University, 2003

\begin{abstract}
A Dissertation presented to the Graduate Faculty of the University of Virginia in Candidacy for the Degree of Doctor of Philosophy
\end{abstract}

\author{
Neuroscience Graduate Program \\ University of Virginia
}

August, 2009 


\section{Abstract}

Serotonin signaling plays a key role in the regulation of development, mood and behavior. Selective serotonin reuptake inhibitors (SSRIs) have been the standard of treatment for several mental disorders, including depression. However, our understanding of their effects is incomplete and the serotonergic system in mammals is complex, making it difficult to study. Drosophila is well suited for the study of the basic mechanisms of serotonergic signaling, but the small size of its nervous system has previously precluded the direct measurements of neurotransmitters. We have developed a novel combination of methods to study serotonergic signaling in the larval Drosophila central nervous system. Fast-scan cyclic voltammetry at inserted microelectrodes is used to detect serotonin elicited by channelrhodopsin-2 (ChR2) mediated depolarization. This dissertation demonstrates the first real-time measurements of serotonin dynamics in a single larval Drosophila nerve cord. A characterization of serotonin release and clearance in the fly, including the estimation of Michaelis-Menten constants, shows that they are analogous to those in mammals, making this simple organism more useful for the study of the basic physiological mechanisms of serotonergic signaling.

The effects of pharmacologically inhibiting serotonin synthesis or reuptake on the releasable pool of serotonin are probed with multiple stimulation experiments. Reuptake is shown to be important for the clearance of serotonin from the extracellular space as well as the rapid replenishment of the releasable pool. Synthesis is critical to the longerterm replenishment of the releasable pool, especially when reuptake is concurrently inhibited. Decreases in serotonin are rescued by inhibiting action potential propagation with tetrodotoxin, implicating endogenous activity in depletion of neuronal serotonin. These results give insight into the possible effects of SSRIs on the serotonergic system 
and the important role that synthesis may play in this phenomenon as well as in overall serotonergic neuron function. The have also paved the way for future use of Drosophila for large-scale genetic analysis of neurotransmitter dynamics. This dissertation was completed under the direction of Jill Venton, $\mathrm{PhD}$, in the Department of Chemistry and Neuroscience Graduate Program. 


\section{Chapter 1. General introduction}

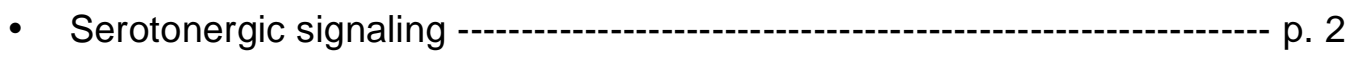

- Polymorphisms affecting serotonergic signaling ----------------------- p. 5

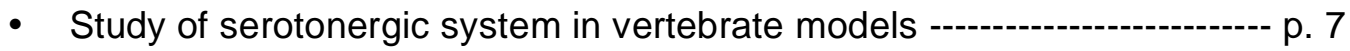

- The fly as a model organism for the study of serotonergic signaling ----- p. 9

- Fast scan cyclic voltammetry and serotonin detection -------------------- p. 13

- Serotonergic signaling in the fly ---

\section{Chapter 2. Diffusion of small molecules into the larval Drosophila CNS}

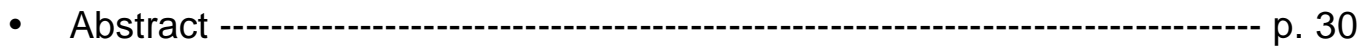

- Introduction -

- Methods

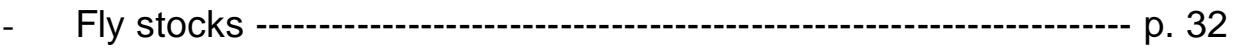

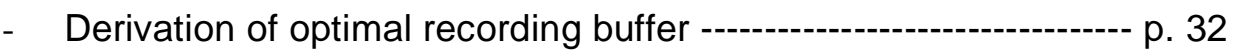

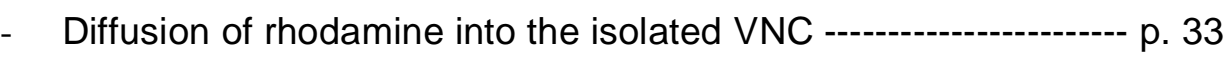

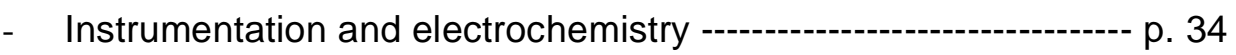

- Preparation of ventral nerve cords (VNCs) for dopamine diffusion

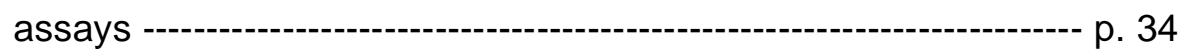

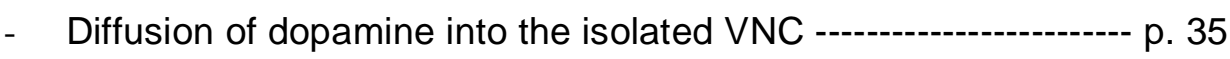

- Results

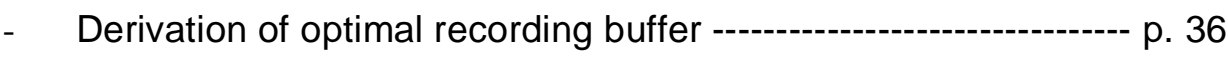

- Diffusion of SR101 into the isolated VNC ----------------- p. 40

- Diffusion of dopamine into the isolated VNC -------------------- p. 42

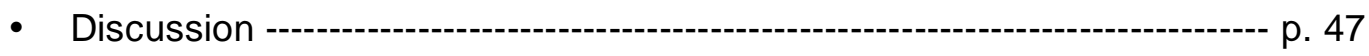

\section{Chapter 3. Quantitative evaluation of serotonin release and clearance in Drosophila}

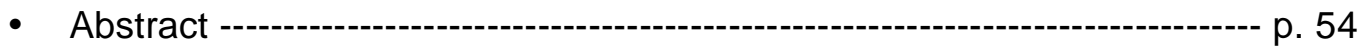

- Introduction --- 55

- Methods

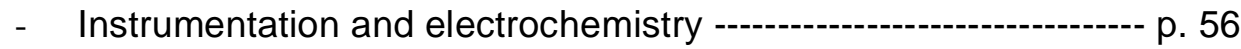




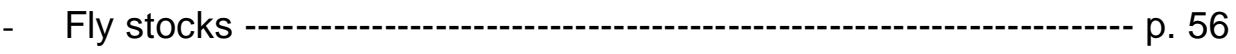

- Preparation of ventral nerve cords (VNCs) ------------- p. 57

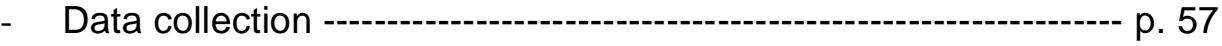

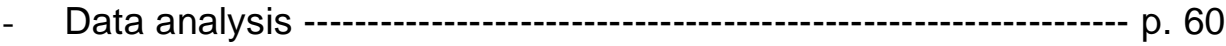

- Results

- Characterization of CHR2-mediated serotonin and dopamine release

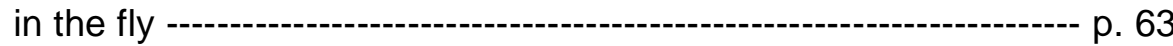

- Release is due to vesicular serotonin ------------------ p. 68

- Characterization of serotonin clearance in the fly ------------------ p. 70

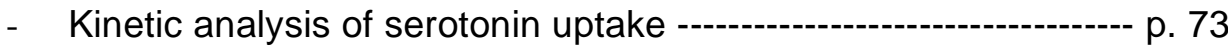

- Discussion

- Serotonin release is vesicular and dependent on serotonin synthesis p. 76

- Characterization of serotonin release in the fly --o- p. 77

- Effect of transporter inhibition on serotonin clearance ------------ p. 78

- Kinetic analysis of serotonin uptake in the fly --- 79

- Conclusions

p. 80

\section{Chapter 4. Serotonin synthesis and reuptake work synergistically to maintain the neuronal serotonin pool in Drosophila.}

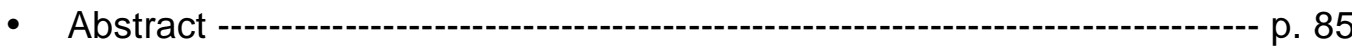

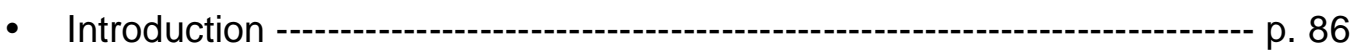

- Methods

- Fly stocks ---

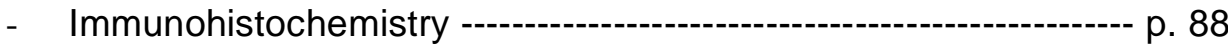

- Instrumentation and electrochemistry ------------------------- p. 88

- Preparation of ventral nerve cords (VNCs) and data collection - p. 89

- Data analysis ------------- 90

- Results

- Neuronal serotonin content after pharmacological manipulations p. 91

- Characterization of stimulated serotonin release in the fly -------- p. 96

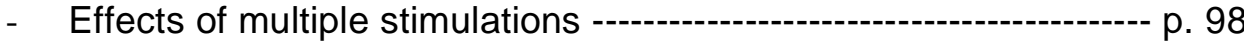


- Effects of reuptake inhibition on releasable pool of serotonin --- p. 99

- Effect of synthesis inhibition on stimulated release p. 105

- Effect of combines synthesis and reuptake inhibition on stimulated release p. 106

- Effect of spontaneous activity on serotonin depletion p. 110

- Discussion

- The releasable serotonin pool in Drosophila is influenced by neuronal activity p. 111

- Reuptake is important for short-term refilling of the releasable pool

- Serotonin synthesis is critical to maintaining the releasable pool

\section{Chapher 5. General discussion}

- The technical challenges of monoamine detection in the fly

- FSCV: Technical considerations p. 120

- Stimulating serotonin release in the fly p. 122

- Serotonin signaling in the fly is analogous to that of mammals p. 123

- Reuptake clears serotonin from the extracellular space and recycled serotonin is rapidly reintegrated into the releasable pool

- Serotonergic neurons maintain serotonin content through synthesis --- p. 126

- Serotonin synthesis and the treatment of depression p. 127

- Future directions

- Chronic SERT inhibition - modeling SSRI treatment p. 129

- Development of exogenously applied 5-HT clearance assay --- p. 131

- The characterization of dopamine release and clearance in larval Drosophila p. 133

- Measuring monoamine release and clearance in adult fly p. 134

- Concluding remarks 


\section{Chapter 1. General introduction}

Major depressive disorder (MDD), with an estimated lifetime prevalence of 1 in 6 individuals, poses a large social and economic cost [1,2]. Current first line treatment for this disorder is the administration of selective serotonin reuptake inhibitors (SSRIs) such as Fluoxetine or Sertaline. Second-line treatments include Monoamine oxidase inhibitors (MAOI), which target the degradation of serotonin. Tricyclic antidepressants and more recently, serotonin - norepinephrine reuptake inhibitors (SNRI) target norepinephrine transporters in addition to SERT. All 4 classes of drugs act to increase extracellular serotonin levels in an effort to alleviate decreases in serotonin thought to be associated with depression. Although the serotonin theory of depression is over 40 years old [3], the link between serotonin signaling and behavioral disorders is still not clear [4-6]. An estimated 30-40\% of people with MDD do not respond to SSRI treatment [7]. Our incomplete understanding of the basic mechanisms of serotonergic signaling further complicates the picture.

Serotonin signaling is involved in the modulation of nearly every neuronal circuit in the brain and its complexity makes it hard to study in mammals [8-12]. The serotonergic system has been highly conserved from humans to $C$. Elegans [13] and can therefore be studied in simple model organisms such as the fruit fly, Drosophila melanogaster. Drosophila boasts a fully developed network of serotonergic neurons within a small, easily accessible, well characterized, and relatively simple central nervous system (CNS). The goal of this work has been to gain a better understanding of the basic mechanisms behind serotonergic signaling in this simple organism and to relate the findings back to more complex systems and clinical data. 


\section{Serotonergic signaling}

Serotonergic signaling plays a role in numerous developmental processes and in adult physiology. Processes such as sleep, memory formation, and even learning have been tied to serotonergic neuron function [14]. In the mammalian CNS serotonergic neurons are located in the dorsal and medial raphe nuclei within the brainstem. They are organized into clusters, sending projections to distinct, partially overlapping areas throughout the cortex and down into the spinal cord (Figure 1) [15]. Tracing studies show that the somatosensory and visual areas of the cortex are innervated by dorsal raphe neurons while motor areas receive input from both dorsal and medial nuclei [16].

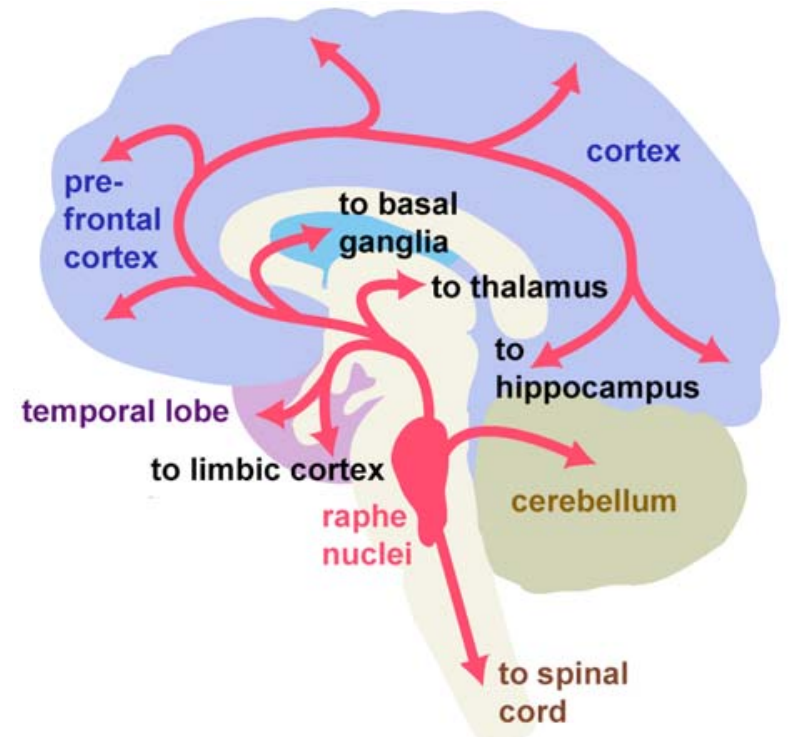

Figure 1: The human serotonergic system. Serotonergic neurons located in the raphe nuclei (red) send projections throughout the cortex, to basal ganglia, thalamus, hippocampus, cerebellum, and down into the spinal cord. Adapted from CNS forum image bank: www.cnsforum.com

A schematic of the main proteins involved in serotonin synthesis, packaging, release and reuptake is shown in Fig 2. Serotonergic neurons synthesize serotonin (5hydroxytryptamine, 5-HT) from the precursor tryptophan. Tryptophan is absorbed from the diet and is then bound to plasma albumin, with only $5 \%$ remaining free for transport to the CNS [17]. Transport across the blood brain barrier is carried out by a large neutral amino acid carrier, which is also responsible for the transport of valine, leucine, isoleusine, methionine, phenylalanine, and tyrosine [18]. The rate-limiting enzyme, 
tryptophan hydroxylase (Tph), converts tryptophan to 5-hydroxytryptophan (5-HTP). Two different isoforms of Tph have been identified. Tph1 discovered first and is expressed primarily in periphery. Tph2, expressed in the CNS, is responsible for the majority of neuronal serotonin synthesis [19]. Under normal conditions Tph2 is only $50 \%$ saturated and synthesis is therefore dependent on tryptophan availability [20]. 5-HTP is rapidly converted to $5-\mathrm{HT}$ by aromatic amino acid decarboxylase also called dopa decarboxylase (ddc).

Serotonin is packaged for neurotransmission by the vesicular monoamine transporter (VMAT). VMAT transports monoamines (5-HT, dopamine, histamine, and noradrenaline) from the cytoplasm into vesicles using a proton gradient. As with Tph, two isoforms have been identified; VMAT2 is expressed in the CNS and VMAT1 in the periphery. Packaged serotonin can then be released by vesicle exocytosis. Serotonergic neurons spontaneously fire at a rate of $\sim 1 \mathrm{~Hz}$ [21], with a sub-population undergoing burst firing $[22,23]$. The functional significance of this is not well understood but implies that the CNS is constantly exposed to the neurotransmitter.

Serotonergic signaling is thought to occur via the neurotransmitter diffusing from the release site through the extracellular space to receptors. This extrasynaptic mechanism of signaling, termed volume transmission has slowly gained acceptance, as evident from a number of recent reviews [24-27]. With the exception of 5-HT3, a serotonin gated ion channel, the rest of the 14 serotonin receptor classes act through $\mathrm{G}$ protein coupled signaling [28]. Consistent with the proposed signaling mechanism, most serotonin receptors are found outside of synaptic specializations [8]. Activation of these high affinity receptors is dependent on extracellular serotonin levels. The actions of serotonin are chiefly limited by SERT, which uses the cross-membrane ion gradient to achieve translocation of serotonin. Along with serotonin, a molecule of $\mathrm{Na}^{+}$and one of 
$\mathrm{Cl}^{-}$are transported in and $\mathrm{K}^{+}$molecule is transported out of the neuron [29]. Recovered serotonin can be recycled via repackaging or degraded.

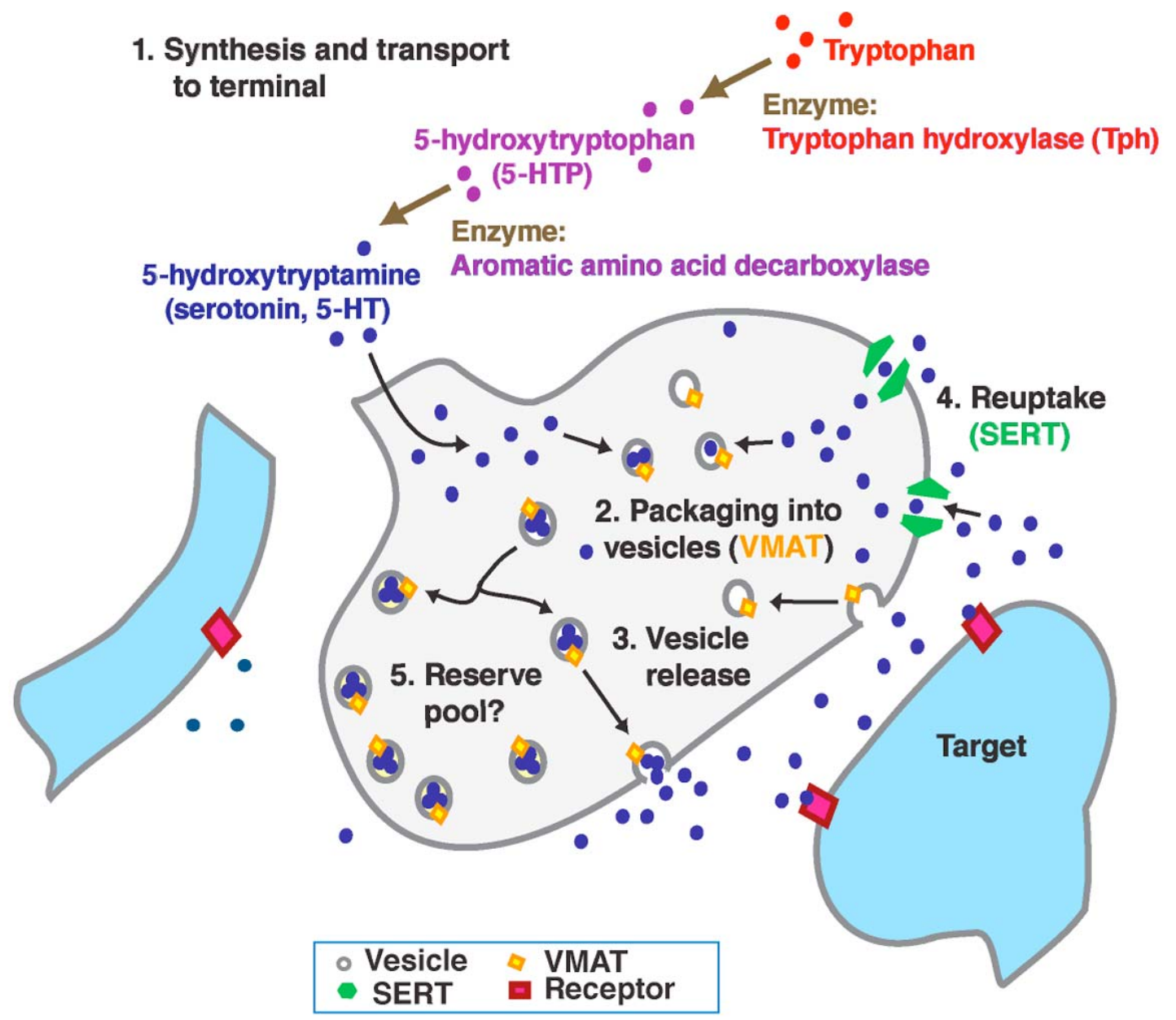

Figure 2. Serotonergic signaling. (1) Serotonin (blue) is synthesized from the precursor tryptophan (red). (2) Serotonin is then packaged into vesicles by VMAT and released (3) during neuronal activity. (4) Released serotonin can diffuse from the release site and bind to receptors. Extracellular serotonin is taken back up by SERT and can then be recycled of degraded. It is unclear how much serotonin remains as a reserve population (5).

Unlike other neurotransmitters like glutamate, serotonin is metabolized only in the cytoplasm and not in the extracellular space. The most common pathway is via monoamine oxidase (MAO) and aldehyde dehydrogenase, which convert 5- HT to 5- 
hydoxyindole acetic acid (5-HIAA). An alternate degradation pathway involves acetylation to $\mathrm{N}$-acetyl serotonin by $\mathrm{SHT} \mathrm{N}$-acetylase and followed by conversion to melatonin by 5 -hydroxy indole-O-methyl transferase [30, 31]. Drugs targeting MAO were commonly used for the treatment of MDD prior to the discovery of SSRIs, which offered fewer drug interactions and reduced side effects. The efficacy of serotonin-based therapies for depression and other psychological illnesses supports the hypothesis that serotonergic signaling plays a role in their pathophysiology. Recently, population genetics has uncovered associations between polymorphisms in serotonin-related proteins and a slew of behavioral characteristics. This controversial data is covered in the next section.

\section{Polymorphisms affecting serotonergic signaling}

Although the development of MDD has been linked to major life stressors, not all people that experience them become depressed. Some individuals may be genetically primed to develop MDD and likewise for other mental disorders. Genetic variation may also affect treatment outcomes and therefore a concerted effort has been made to identify and characterize the alleles involved. The human genome is diverse, with over 4 million single nucleotide polymorphisms identified [32]. An even greater number of base pair changes are thought to exist as a result of insertions, deletions, and inversions in the genome [33,34]. Numerous polymorphisms in serotonin-related proteins have been characterized including those in Tph, MAOA, VMAT, and SERT. Although several Tph2 polymorphisms have been identified in the general population [35-38], their prevalence and possible association with behavioral phenotypes as well as response to antidepressant treatment has been subject to considerable controversy [39-41]. However, a recent meta analysis confirms the presence of a strong association with suicidal behavior [42]. MAO polymporphisms affecting serotonin levels are associated 
with increased propensity towards violence [43] and MDD [44]. VMAT polymorphisms have not yet been well characterized, but preliminary studies have shown an association with depression in the elderly [45] and with alcohol dependence, especially in males [46, 47].

Polymorphisms in SERT, especially its promoter region, are the best characterized of the group. Two main promoter alleles were originally identified. The more prevalent, long form (L), conferring normal SERT expression and the short allele (s), conferring reduced SERT expression [48]. The short form is associated with an increased susceptibility to elevated anxiety, neuroticism [49], depressive symptoms refractory to SSRI treatment [7,50-52], and increased susceptibility to acute tryptophan depletion [53-55] A meta-analysis has confirmed the association of low-functioning SERT polymorphisms with effectiveness of SSRI treatment [56].

Recently, the long allele has been further subdivided into La and Lg subtypes to account for a sub-allele, La, which conferred short form-like activity [57]. Homozygosity for the La form has been linked to decreased hippocampal volumes in depressed patients [58]. Because previous studies had included La individuals in the normal function group, the effect of lower SERT expression may have been underestimated [59]. Decreased SERT expression appears to predispose individuals to the development of depression in the face of other challenges to the serotonergic system as opposed to directly leading to the disorder [60]. The lack of a direct link between polymorphisms and disease states suggests that these disorders are multi-factorial and more work is necessary to understand the interplay between environmental and genetic variables. Model organisms provide key opportunities for increasing our understanding of these interactions. 


\section{Study of serotonergic system in vertebrate models}

Rodent models are widely utilized in the study of serotonin because they can provide data ranging from molecular details to behavioral phenotypes. In mice, a traditional approach has been to selectively eliminate or knock out a gene and then evaluate the phenotype of resulting progeny. Although precisely modeling human behavioral traits in rodents is difficult, performance on a number of tests is thought to be correlated with levels of aggression, anxiety, and/or depression.

Tph1 and Tph2 have been knocked out in mice, recently in combination [61]. Surprisingly, these mice display no gross anatomical deficits and only mild behavioral phenotypes including anxiety and depressive behavior. Despite the knockout of both Tph isoforms, small amounts of serotonin are present in the CNS throughout life. The phenotype of these rodents may have been ameliorated by compensation through

alternate synthesis pathways such as phenylalanine hydroxylase (PAH). Conversely, when serotonin synthesis is inhibited in developing rat through the application of PCPA, which also inhibits $\mathrm{PAH}$ and decreases maternal serotonin, long-lasting structural changes in the somatosensory cortex are identified [62]. This suggests that during development, many redundant sources of serotonin exist and highlights the importance of serotonin in these processes.

Packaging of serotonin by VMAT is critical for serotonergic signaling. VMAT2 knockout mice are unable to package monoamines into synaptic vesicles, resulting in a loss of monoamine signaling $[63,64]$. Despite grossly normal neuronal early development likely due to maternal monoamines [65], mice homozygous for the knockout die soon after birth. Heterozygotes display a depressive-like phenotype [66], supporting a causative relationship between low serotonin levels and depression. Fluorescence-based imaging shows that cultured neurons from VMAT KO mice continue to endo and exocytose vesicles despite a lack of neurotransmitter content. Therefore 
VMAT2 activity affects the number of functional vesicles as opposed to the total vesicle population [65].

As with SERT polymorphisms in humans, SERT knockouts have been the subject of the bulk of research in this field. Two SERT knockout mice strains have been independently generated $[67,68]$. SERT KO mice exhibit four to six-fold increases in extracellular serotonin concentration $[69,70], 50 \%$ decreases in serotonin tissue concentration [71], alterations in sensory patterning, compensatory changes in receptor expression [72], and long-term behavioral and sleep pattern abnormalities.

Morphologically, the glutamatergic thalamocortical neurons innervating the primary somatosensory, visual, and auditory areas of the cortex appear to be the most sensitive to changes in serotonin levels [73]. Perturbations in this neuronal population in the mouse are most apparent in the barrel field area, where inputs from each whisker of the snout normally map onto a precise cluster of projections corresponding to it $[74,75]$. In SERT knockout mice, the barrels are disrupted, leaving a jumble of overlapping fields along with decreased axonal branching. Additional patterning defects are seen in the segregation of retinal ganglion cell projections into areas of the retinogeniculate nucleus and the superior colliculus.

SERT knockout mice exhibit decreased levels of aggression [76]. Other behavioral changes appear to be strain-dependent implicating modifier loci. Some mice show increased levels of anxiety-like behaviors, with minor stressors causing exaggerated adrenocorticotropic and catecholamine release [77]. Others exhibit a depression-like phenotype, characterized by decreased attempts to escape unpleasant stimuli [67] and increased stress-sensitivity. Knockout mice exhibit decreased performance on many of the same tasks normally improved by SERT blockade in adulthood, suggesting that their behavioral deficiencies result from aberrant development $[78,79]$. SSRI treatment during the mouse and rat early postnatal periods 
produces a phenotype similar to those seen in SERT KO mice, confirming the presence of a developmental effect [80]. Treated animals show a permanent reduction in Tph in the dorsal raphe and SERT expression in the cortex as well as increased anxiety-like behavior and an impairment of shock avoidance [81-85]. Additional defects are seen in sexual competence/activity, alterations in locomotor activity, and REM sleep regulation. None of these SSRI induced alterations are observed in adults undergoing similar type and duration of treatment, reinforcing the idea of a difference in sensitivity across development.

MAOA knockouts, similar to SERT knockouts, show developmental defects in thalamocortical and retinal projections. MAOA knockout mice show a nine-fold increase in extracellular serotonin during early development. Although working through different mechanisms, both cause increased serotonin levels during development, further demonstrating the importance of regulated extracellular serotonin levels in activitydependent cortical patterning and the evolution of behavior.

This section has primarily focused on genetic knockouts of proteins involved in serotonergic signaling. Although valuable, knockouts tend to present a highly complex phenotype due to developmental effects as well as compensatory changes made throughout the organism's lifetime. Acute pharmacological studies help clarify some of the phenotype observed but the system is still highly complex and it is difficult to establish causality. Simpler model organisms such as the fruit fly are especially useful for this purpose.

\section{The fly as a model organism for the study of serotonin signaling}

At room temperature the fly egg hatches into a $1^{\text {st }}$ instar (L1) larva within $24 \mathrm{~h}$ of being laid. Over the next 2 days it molts into a $2^{\text {nd }}$ and then $3^{\text {rd }}$ instar (L2 and L3, respectively). At around day 6 the larva undergoes pupation. The adult emerges 4 days 
later and can initiate egg laying after just 8 more hours [86]. Consequently, genetic mutants in the fly can be produced in as little as a month while similar manipulations can take years to accomplish in rodents. Because of its rapid lifecycle and fully sequenced genome [87], the fly is well suited for large-scale genetic screens. A recent quantitative trait loci (QTL) screen identified 13 genes responsible for variability in locomotor reactivity, including dopa decarboxylase. Hundreds of flies from 96 lines were screened and broadly mapped QTLs were narrowed by complementation studies using flies deficient for portions of the genome [88]. Such an approach would not be feasible in mammals due to time and cost.

Drosophila exhibit complex behaviors, such as sleep [89], circadian rhythms[90], learning and memory [91, 92], aggression [93],courtship [94], feeding [95], and stereotyped responses to drugs of abuse that are relevant to higher order organisms [96, 97]. For example, territory fighting rate in flies is increased by administration of 5-HTP or overexpression of Tph, demonstrating the role of serotonin in aggression [98]. Genetic or pharmacological elevation of serotonin also increases the amount of time flies sleep. Further genetic dissection of this phenotype revealed that it depended on $5 \mathrm{HT}-1 \mathrm{~A}$ receptor expression in the mushroom body region of the brain [99]. The techniques used to gain spatio-temporal control over gene expression in this study represent yet another strength of the fly, a large repository of genetic tools.

Flybase, an online database of Drosophila genes and genomes provides an invaluable resource by compiling a wide variety of tools with links to fly repositories and additional private data. There are several public repositories of genetically altered flies, including the rather large Bloomington stock center located in Bloomigton, Indiana. The genetically altered fly library includes hundreds of lines from previous mutagenesis screens as well as many deletions created by excising previously created viral 
insertions. These resources greatly increase the ease with which screens can be conducted.

One important tool available in Drosophila is the UAS-GAL4 targeting system (Figure 3), which is a common tool for achieving spatial and temporal control over gene expression. The yeast upstream activating system (UAS) serves as a promotor for inserted genetic elements. Wherever GAL4 protein is expressed, UAS is activated and the inserted gene is transcribed. By altering the promotor linked to GAL4, the expression of UAS can be targeted to specific cell type at a specific time [100]. Similar control of expression has been achieved in mammals by means of the FLP-FRT system, although this system is also available for use in the fly.

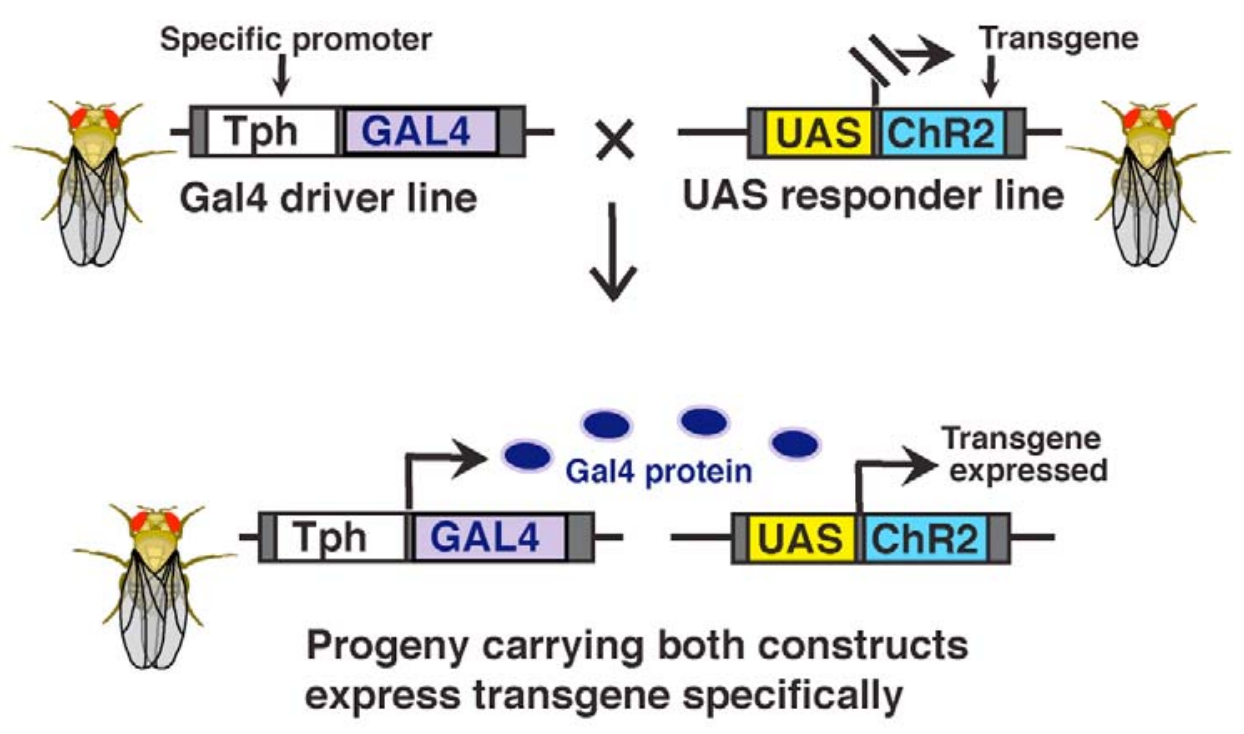

Figure 3. The GAL4-UAS system in the fly. Flies containing the GAL4 driver line are crossed to others carrying a transgene linked to UAS. Resulting progeny express the transgene in a spatio-temporal pattern dictated by the promotor used to drive GAL4 expression.

Drosophila provides much of the simplicity and ease of manipulation of cell culture within an intact developed nervous system. The fly serotonergic system is 
homologous to that of mammals but is simpler, containing 100 individually identifiable neurons as opposed to over 20,000 neurons in mice, or 300,000 in humans. About $75 \%$ of human genes have functional orthologs that have been identified in Drosophila [101]. For example, Drosophila express SERT and four serotonin receptor subtypes that are similar to their human orthologs [102]. The metabolic pathway by which serotonin is degraded in the fly remains controversial, although recent data suggests that serotonin metabolism occurs through acetylation [103].
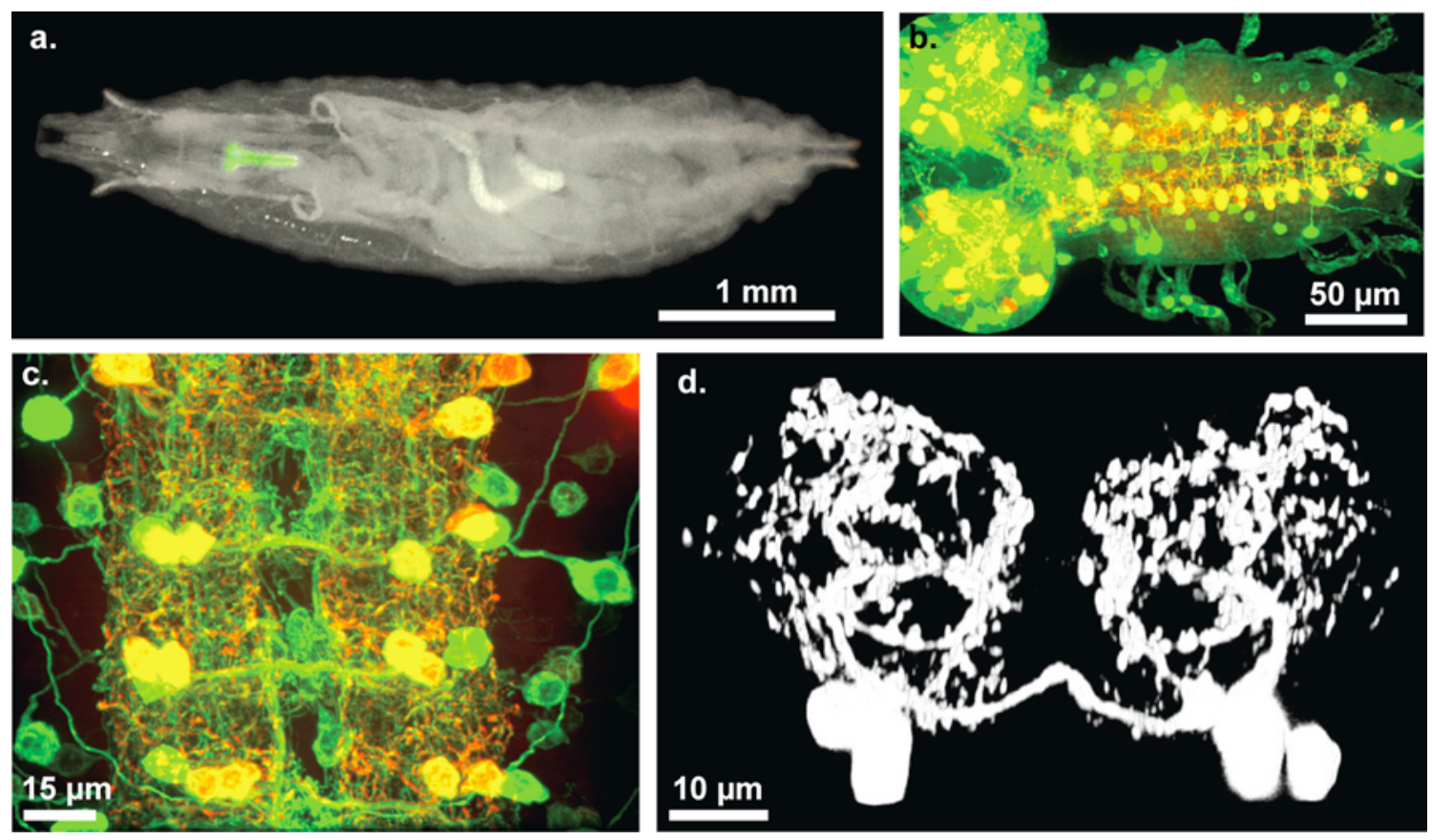

Figure 4. The fly sertonergic system. (a) L3 larva expressing GFP in the CNS, reprinted from www.salk.edu. (b) Isolated CNS from an L3 larva expressing GFP in serotonergic and dopaminergic neurons, stained for GFP (green) and serotonin (red). Serotonergic neurons can be seen as paired yellow cells running down the length of the ventral nerve cord (right) and throughout the optic lobes (left). (c) Larger magnification image of the stained ventral nerve cord showing abdominal segments in greater detail. (d) Reconstruction of an abdominal segment in the y plane showing the complexity of the serotonergic neuron arborizations. 
The development and morphology of the serotonergic system in the fly has been well characterized [104-107]. The fly serotonergic system (Figure 4) consists of several small clusters of neurons in the brain and paired neurons within each hemisegment of the ventral nerve cord (VNC), a segmental ganglion analogous to our spinal cord [105]. Each neuron sends its axons across the midline to the contralateral side of the VNC, where they arborize within the neuropil into a highly stereotyped pattern recently described by J. Chen [104]. The established patterns are maintained throughout the larval stage, undergoing reorganization into the adult pattern during pupation. The density of serotonergic terminals is regulated by serotonergic activity and can be decreased by exogenous application of serotonin [108] or increased by inhibiting serotonergic neuron activity by blocking vesicle endocytosis (J. Chen, $\mathrm{PhD}$ thesis). Previous work has therefore provided a lot of information on serotonergic neuron structure and how it is influenced by internal and external factors. However, serotonergic signaling itself has not been investigated in the fly due to a lack of detection techniques.

\section{Fast scan cyclic voltammetry and serotonin detection}

Two main categories of detection techniques have been utilized to measure serotonin levels, imaging and direct detection of extracellular levels. Imaging allows for the monitoring of intracellular serotonin in real time, but provides only a rough estimate of serotonin content and is not reliable for measuring extracellular serotonin levels [109]. Direct detection of monoamines can be achieved through microdialysis or electrochemistry and allows for precise measurement of serotonin content with varying spatial and temporal resolution, depending on the technique used. Microdialysis offers the advantage of detecting many different molecules at the same time, while electrochemistry at microelectrodes offers increased spatio-temporal resolution. 
Microdialysis involves the continuous sampling of the extracellular fluid using a hollow fiber dialysis membrane attached to a cannula with inlet and outlet tubes with continuous flow-through of iso-osmolar physiological fluid at a rate of 0.1 to $2 \mu \mathrm{l} / \mathrm{min}$ [110]. Analyte diffuses down the concentration gradient through the membrane into the sample fluid. The dialysate is then analyzed using high performance liquid chromatography (HPLC) or capillary electrophoresis (CE). Molecules are separated by size or charge and identified by their elution times. The acquisition of more than one measurement per minute is rare because time resolution is limited by cross-membrane diffusion and dialyte accumulation (several $\mu \mathrm{l}$ necessary) [111].

Because the probe tip is larger than the fly CNS, microdialysis is not feasible in the fly. Instead, pooled homogenate tissue from dissected brains or whole heads is analyzed by CE or HPLC [112]. The analysis of homogenate can provide critical data on the overall levels of the monoamines and their metabolites. For example, HPLC analysis showed that total brain serotonin levels decrease following administration of the serotonin synthesis inhibitor, p-chlorophenylalanine [113]. CE has provided clues to how serotonin is metabolized in the fly. Ewing and colleagues found abundant $\mathrm{N}$-acetylated serotonin metabolites but did not detect any 5-HIAA in brain homogenates. This suggests that in the fly serotonin is metabolized by primarily by acetylation as opposed to degradation by MAO [103].

Although excellent for measuring overall levels of multiple analytes at the same time, CE and HPLC have significant drawbacks in the fly. Repeat measurements cannot be performed in the same fly, preventing the acquisition of a time-course from a single animal. The time resolution is not high enough to allow the measurement of rapid changes in serotonin levels. Tissue homogenization is an additional barrier to the detection of serotonin signaling because it occurs through the translocation of neurotransmitter from the intracellular to the extracellular space without changing overall 
levels. In order to directly measure serotonin signaling in the fly, greater spatio-temporal resolution is necessary.

Electrochemical detection is based on the work of Herovsky, who in 1924 discovered that the current at an electrode consisted of two components, the charging current and an additional current due to oxidation/reduction of electroactive species, the Faradaic current [114]. The charging current results from the charging of the electrode surface and is an intrinsic property of the material used to make the electrode and the solution surrounding the electrode. The Faradaic current results from the summation of all oxidation/reduction events at the electrode surface. Techniques utilizing carbon fiber electrodes to measure electroactive species in vivo were developed several decades later, gaining increasing popularity by the mid 1980s [114]. A major limitation of using electrodes to measure the concentration of a particular electroactive species is that the background current tends to be much larger than the signal resulting from the oxidation or reduction of the analyte. The background current includes the charging current as well as signal from other electroactive species present near the electrode surface. As a result of this limitation, electrochemical detection techniques are best suited to identifying changes in neurotransmitter levels as opposed to measuring basal levels.

A wide range of electrochemical techniques has been developed, including constant potential amperometry, chronoamperometry and fast scan cyclic voltammetry (FSCV). Constant potential amperometry involves holding the electrode potential at a value high enough to oxidize the species of interest. It offers the most rapid time resolution. However, constant potential amperometry provides little chemical selectivity. Chronoamperometry employs a voltage pulse, in which the potential at the electrode is instantaneously increased to right above the oxidation potential of the species under investigation and held there for a short time. The current is measured towards the end of the pulse, which minimizes effects due to charging current, and gives an accurate 
measurement of the concentration of the compounds oxidized at this potential. Chronoamperometry provides some chemical selectivity. It provides very rapid time resolution with high sensitivity and can be used to measure single vesicle release [115, 116]. Because chronoamperometry offers no additional information to help verify the identity species of interest [117] it is difficult to utilize in vivo. One can circumvented this limitation by applying exogenous neurotransmitter, but applied neurotransmitter amounts tend to exceed the physiological range and the volume utilized can distort local tissue architecture [70, 118, 119].

FSCV provides the most chemical selectivity. A triangular waveform is applied to the electrode, linearly scanning its potential past the oxidation and back past the reduction potentials of the substance of interest (Figure 5). Current is measured throughout the sweep. The electrode spends very little time at potentials sufficient to oxidize or reduce the substance of interest. FSCV is especially useful for serotonin detection because unstable serotonin oxidation products, which can foul electrodes, are generated only briefly prior to being reduced. The holding potential, scan rate, time between scans, and potential limits influence the sensitivity and selectivity of measurements. Every measurement consists of a cyclic voltammogram (CV), a plot of current vs. voltage [117]. The shape of the CV is determined by the electron transfer kinetics and adsorption to the electrode. A large background current, which is proportional to the scan rate and capacitance of the CFE, results from the charging and rearrangement of the electroactive species in the solution. This current is temporally stable so small changes in current due to the species of interest can be detected by means of background subtraction.

A background subtracted CV allows the identification of the species responsible for the signal. Features such as the relative size, shape and locations of oxidation and reduction peaks can be used to aid in species identification. The major advantage of 
FSCV over chronoamperometry is the ability to identify the electroactive species based on its "chemical signature". While providing chemical selectivity, FSCV maintains a high temporal resolution with measurements performed every $100 \mathrm{~ms}$. Because of it's good spatial and temporal resolution, small size, and chemical selectivity, FSCV at carbonfiber microelectrodes is the ideal candidate technique for the study of serotonergic signaling in the fly.
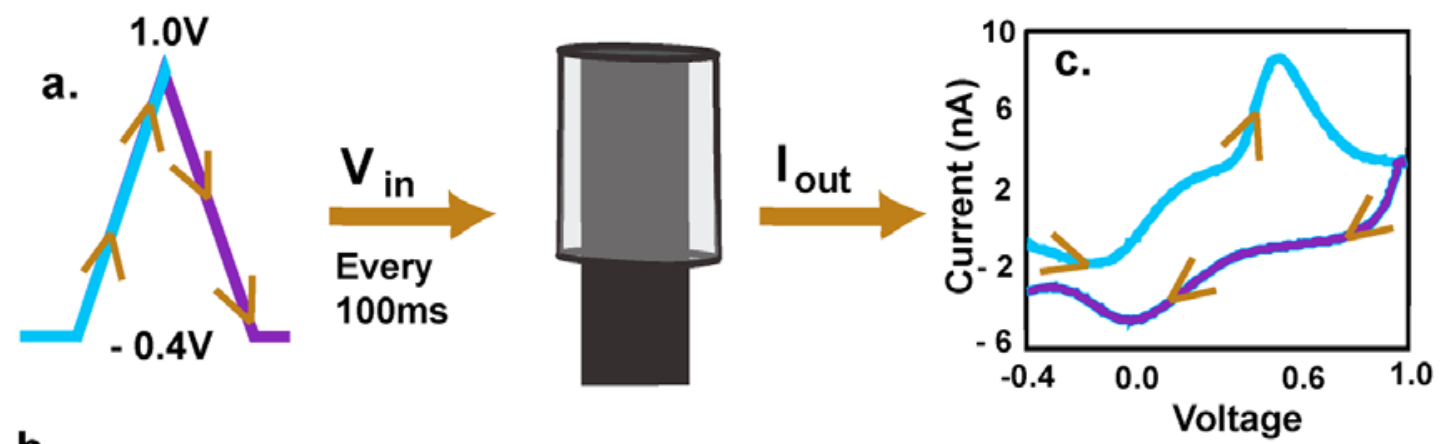

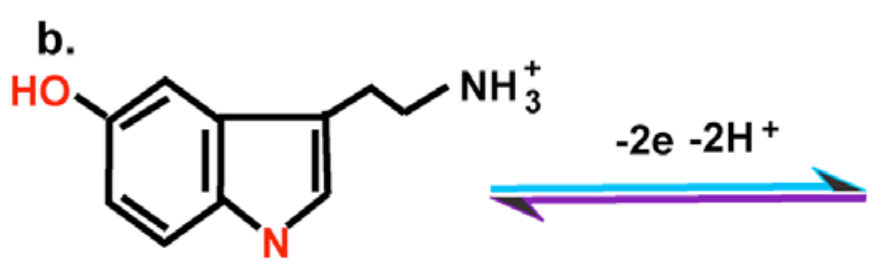

$\mathrm{H}$

\section{Serotonin}

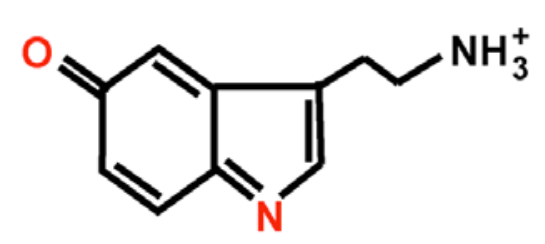

Serotonin-p-quinone

Figure 5. Voltammetric detection of serotonin. (a) A triangular voltage pulse is applied to the electrode every $100 \mathrm{~ms}$. The potential of the electrode is scanned upwards (turquoise) taking the potential of the electrode past the oxidation potential of serotonin. (b) Serotonin is oxidized to the $p$-quinone imine and a positive current is detected by the electrode. The potential is then rapidly decreased (purple), reducing the newly-oxidized serotonin resulting in a negative current. (c) The peaks in current can be seen on the background subtracted cyclic voltammogram. The arrows on this diagram correspond to those on the electrode voltage diagram to show the temporal progression of the acquired signal. 


\section{Serotonergic signaling in the fly}

This dissertation describes the development of a technique to measure real time serotonin release and clearance in the larval Drosophila VNC. The next chapter focuses on the initial optimization of FSCV for use in the fly, including the development of buffer composition, dissection protocol, and diffusion-based pharmacological assays. The third chapter describes the implementation of a genetic approach to induce and quantify endogenous serotonin or dopamine release. This approach provides the first measurements of serotonergic signaling in larval Drosophila and allows approximation of the Michaelis-Menten uptake constants for SERT in the fly. Comparison of measured and previously published values to shows that serotonergic signaling in the fly is analogous to that of mammals. The final chapter builds on the initial characterization of serotonergic signaling, exploring the mechanisms by which serotonergic neurons maintain their signaling capacity. The results directly show that serotonin synthesis plays a key role in the function of serotonergic neurons. Synthesis is especially critical when reuptake is inhibited, as occurs during SSRI treatment. This work expands our understanding of the basic mechanisms of serotonergic signaling and adds to the utility of Drosophila for the study of monoamine signaling. 


\section{References:}

1. Kessler, R.C., et al., The epidemiology of major depressive disorder: results from the National Comorbidity Survey Replication (NCS-R). JAMA, 2003. 289(23): p. 3095-105.

2. Kessler, R.C., et al., Prevalence and effects of mood disorders on work performance in a nationally representative sample of U.S. workers. Am J Psychiatry, 2006. 163(9): p. 1561-8.

3. Coppen, A., The biochemistry of affective disorders. Br J Psychiatry, 1967. 113(504): p. 1237-64.

4. Harmer, C.J., Serotonin and emotional processing: does it help explain antidepressant drug action? Neuropharmacology, 2008. 55(6): p. 1023-8.

5. Vergne, D.E. and C.B. Nemeroff, The interaction of serotonin transporter gene polymorphisms and early adverse life events on vulnerability for major depression. Curr Psychiatry Rep, 2006. 8(6): p. 452-7.

6. Moltzen, E.K. and B. Bang-Andersen, Serotonin reuptake inhibitors: the corner stone in treatment of depression for half a century--a medicinal chemistry survey. Curr Top Med Chem, 2006. 6(17): p. 1801-23.

7. Smits, K.M., L.J.M. Smits, J.S.A.G. Schouten, F.P.M.L. Peeters, and M.H. Prins, Does pretreatment testing for serotonin transporter polymorphisms lead to earlier effects of drug treatment in patients with major depression? A decision-analytic model. Clinical Therapeutics, 2007. 29(4): p. 691-702.

8. Barnes, N.M. and T. Sharp, A review of central 5-HT receptors and their function. Neuropharmacology, 1999. 38(8): p. 1083-152.

9. Vitalis, T. and J.G. Parnavelas, The role of serotonin in early cortical development. Dev Neurosci, 2003. 25(2-4): p. 245-56.

10. Jacobs, B.L. and E.C. Azmitia, Structure and function of the brain serotonin system. Physiol Rev, 1992. 72(1): p. 165-229.

11. Gaspar, P., O. Cases, and L. Maroteaux, The developmental role of serotonin: news from mouse molecular genetics. Nat Rev Neurosci, 2003. 4(12): p. 100212.

12. Haydon, P.G., D.P. McCobb, and S.B. Kater, The regulation of neurite outgrowth, growth cone motility, and electrical synaptogenesis by serotonin. J Neurobiol, 1987. 18(2): p. 197-215. 
13. Hen, R., Structural and functional conservation of serotonin receptors throughout evolution. Exs, 1993. 63: p. 266-78.

14. Gonzalez-Burgos, I. and A. Feria-Velasco, Serotonin/dopamine interaction in memory formation. Prog Brain Res, 2008. 172: p. 603-23.

15. Hornung, J.P., The human raphe nuclei and the serotonergic system. J Chem Neuroanat, 2003. 26(4): p. 331-43.

16. Dori, I., A. Dinopoulos, M.E. Blue, and J.G. Parnavelas, Regional differences in the ontogeny of the serotonergic projection to the cerebral cortex. Exp Neurol, 1996. 138(1): p. 1-14.

17. McMenamy, R.H., Binding of indole analogues to human serum albumin. Effects of fatty acids. J Biol Chem, 1965. 240(11): p. 4235-43.

18. Hood, S.D., C.J. Bell, and D.J. Nutt, Acute tryptophan depletion. Part I: rationale and methodology. Aust N Z J Psychiatry, 2005. 39(7): p. 558-64.

19. Coleman, C.M. and W.S. Neckameyer, Serotonin synthesis by two distinct enzymes in Drosophila melanogaster. Arch Insect Biochem Physiol, 2005. 59(1): p. 12-31.

20. Schaechter, J.D. and R.J. Wurtman, Serotonin release varies with brain tryptophan levels. Brain Res, 1990. 532(1-2): p. 203-10.

21. Zhang, Q.J., R. Gao, J. Liu, Y.P. Liu, and S. Wang, Changes in the firing activity of serotonergic neurons in the dorsal raphe nucleus in a rat model of Parkinsonos disease. Sheng Li Xue Bao, 2007. 59(2): p. 183-189.

22. Rouchet, N., O. Waroux, C. Lamy, L. Massotte, J. Scuvee-Moreau, J.F. Liegeois, and V. Seutin, SK channel blockade promotes burst firing in dorsal raphe serotonergic neurons. Eur J Neurosci, 2008. 28(6): p. 1108-15.

23. Gartside, S.E., E. Hajos-Korcsok, E. Bagdy, L.G. Harsing, Jr., T. Sharp, and M. Hajos, Neurochemical and electrophysiological studies on the functional significance of burst firing in serotonergic neurons. Neuroscience, 2000. 98(2): p. 295-300.

24. Coggan, J.S., et al., Evidence for ectopic neurotransmission at a neuronal synapse. Science, 2005. 309(5733): p. 446-51.

25. Agnati, L.F., G. Leo, A. Zanardi, S. Genedani, A. Rivera, K. Fuxe, and D. Guidolin, Volume transmission and wiring transmission from cellular to molecular networks: history and perspectives. Acta Physiologica, 2006. 187(1-2): p. 329344. 
26. Vizi, E.S. and A. Mike, Nonsynaptic receptors for GABA and glutamate. Curr Top Med Chem, 2006. 6(10): p. 941-8.

27. Arbuthnott, G.W. and J. Wickens, Space, time and dopamine. Trends in Neurosciences, 2007. 30(2): p. 62-69.

28. Hannon, J. and D. Hoyer, Molecular biology of 5-HT receptors. Behav Brain Res, 2008. 195(1): p. 198-213.

29. Rudnick, G., Structure/function relationships in serotonin transporter: new insights from the structure of a bacterial transporter. Handb Exp Pharmacol, 2006(175): p. 59-73.

30. Mefford, I.N., P. Chang, D.C. Klein, M.A. Namboodiri, D. Sugden, and J. Barchas, Reciprocal day/night relationship between serotonin oxidation and $\mathrm{N}$ acetylation products in the rat pineal gland. Endocrinology, 1983. 113(5): p. 1582-1586.

31. Adell, A., A. Carceller, and F. Artigas, In vivo brain dialysis study of the somatodendritic release of serotonin in the Raphe nuclei of the rat: effects of 8 hydroxy-2-(di-n-propylamino)tetralin. J Neurochem, 1993. 60(5): p. 1673-81.

32. Kidd, J.M., et al., Mapping and sequencing of structural variation from eight human genomes. Nature, 2008. 453(7191): p. 56-64.

33. Wong, K.K., et al., A comprehensive analysis of common copy-number variations in the human genome. Am J Hum Genet, 2007. 80(1): p. 91-104.

34. Redon, R., et al., Global variation in copy number in the human genome. Nature, 2006. 444(7118): p. 444-54.

35. Zhang, X., et al., Loss-of-function mutation in tryptophan hydroxylase-2 identified in unipolar major depression. Neuron, 2005. 45(1): p. 11-6.

36. Zhou, Z., et al., Haplotype-based linkage of tryptophan hydroxylase 2 to suicide attempt, major depression, and cerebrospinal fluid 5-hydroxyindoleacetic acid in 4 populations. Arch Gen Psychiatry, 2005. 62(10): p. 1109-18.

37. Zill, P., A. Buttner, W. Eisenmenger, H.J. Moller, B. Bondy, and M. Ackenheil, Single nucleotide polymorphism and haplotype analysis of a novel tryptophan hydroxylase isoform (TPH2) gene in suicide victims. Biol Psychiatry, 2004. 56(8): p. 581-6.

38. Maron, E., et al., Association study of tryptophan hydroxylase 2 gene polymorphisms in panic disorder. Neurosci Lett, 2006. 
39. Tsai, S.J., Y.C. Wang, J.Y. Chen, and C.J. Hong, Allelic variants of the tryptophan hydroxylase $(A 218 C)$ and serotonin $1 B$ receptor $(A-161 T)$ and personality traits. Neuropsychobiology, 2003. 48(2): p. 68-71.

40. Haghighi, F., et al., Genetic architecture of the human tryptophan hydroxylase 2 Gene: existence of neural isoforms and relevance for major depression. Mol Psychiatry, 2008.

41. Mann, J.J., et al., No association between a TPH2 promoter polymorphism and mood disorders or monoamine turnover. J Affect Disord, 2008. 106(1-2): p. 11721.

42. Li, D. and L. He, Meta-analysis supports association between serotonin transporter (5-HTT) and suicidal behavior. Mol Psychiatry, 2006. 12(1): p. 47-54.

43. Meyer-Lindenberg, A., et al., Neural mechanisms of genetic risk for impulsivity and violence in humans. Proc Natl Acad Sci U S A, 2006. 103(16): p. 6269-74.

44. Du, L., D. Bakish, A. Ravindran, and P.D. Hrdina, MAO-A gene polymorphisms are associated with major depression and sleep disturbance in males. Neuroreport, 2004. 15(13): p. 2097-101.

45. Christiansen, L., Q. Tan, M. Iachina, L. Bathum, T.A. Kruse, M. McGue, and K. Christensen, Candidate Gene Polymorphisms in the Serotonergic Pathway: Influence on Depression Symptomatology in an Elderly Population. Biological Psychiatry, 2007. 61(2): p. 223-230.

46. Schwab, S.G., et al., Association of DNA Polymorphisms in the Synaptic Vesicular Amine Transporter Gene (SLC18A2) with Alcohol and Nicotine Dependence. Neuropsychopharmacology, 2005. 30(12): p. 2263-2268.

47. Lin, Z., D. Walther, X.-Y. Yu, S. Li, T. Drgon, and G.R. Uhl, SLC18A2 promoter haplotypes and identification of a novel protective factor against alcoholism. Human Molecular Genetics, 2005. 14(10): p. 1393-1404.

48. Brown, S.M. and A.R. Hariri, Neuroimaging studies of serotonin gene polymorphisms: exploring the interplay of genes, brain, and behavior. Cogn Affect Behav Neurosci, 2006. 6(1): p. 44-52.

49. Caspi, A., et al., Influence of life stress on depression: moderation by a polymorphism in the 5-HTT gene. Science, 2003. 301(5631): p. 386-9.

50. Serretti, A., M. Kato, D. De Ronchi, and T. Kinoshita, Meta-analysis of serotonin transporter gene promoter polymorphism (5-HTTLPR) association with selective 
serotonin reuptake inhibitor efficacy in depressed patients. Mol Psychiatry, 2006. 12(3): p. 247-257.

51. Kang, R.-H., M.-L. Wong, M.-J. Choi, J.-W. Paik, and M.-S. Lee, Association study of the serotonin transporter promoter polymorphism and mirtazapine antidepressant response in major depressive disorder. Progress in NeuroPsychopharmacology and Biological Psychiatry, 2007. 31(6): p. 1317-1321.

52. Hu, X.-Z., et al., Association Between a Functional Serotonin Transporter Promoter Polymorphism and Citalopram Treatment in Adult Outpatients With Major Depression. Archives of General Psychiatry, 2007. 64(7): p. 783-792.

53. Roiser, J.P., U. Muller, L. Clark, and B.J. Sahakian, The effects of acute tryptophan depletion and serotonin transporter polymorphism on emotional processing in memory and attention. Int J Neuropsychopharmacol, 2007. 10(4): p. 449-61.

54. Roiser, J.P., A.D. Blackwell, R. Cools, L. Clark, D.C. Rubinsztein, T.W. Robbins, and B.J. Sahakian, Serotonin transporter polymorphism mediates vulnerability to loss of incentive motivation following acute tryptophan depletion.

Neuropsychopharmacology, 2006. 31(10): p. 2264-72.

55. Neumeister, A., et al., Association between serotonin transporter gene promoter polymorphism (5HTTLPR) and behavioral responses to tryptophan depletion in healthy women with and without family history of depression. Arch Gen Psychiatry, 2002. 59(7): p. 613-20.

56. Lasky-Su, J.A., S.V. Faraone, S.J. Glatt, and M.T. Tsuang, Meta-analysis of the association between two polymorphisms in the serotonin transporter gene and affective disorders. Am J Med Genet B Neuropsychiatr Genet, 2005. 133(1): p. 110-5.

57. Hu, X., G. Oroszi, J. Chun, T.L. Smith, D. Goldman, and M.A. Schuckit, An expanded evaluation of the relationship of four alleles to the level of response to alcohol and the alcoholism risk. Alcohol Clin Exp Res, 2005. 29(1): p. 8-16.

58. Thomas Frodl, P.Z.T.B.C.S.I.R.R.T.Z.B.B.M.R.H.-J.r.M.E.M.M., Reduced hippocampal volumes associated with the long variant of the tri- and diallelic serotonin transporter polymorphism in major depression. American Journal of Medical Genetics Part B: Neuropsychiatric Genetics, 2008. 9999(9999): p. n/a. 
59. Firk, C. and C.R. Markus, Differential effects of 5-HTTLPR genotypes on mood, memory, and attention bias following acute tryptophan depletion and stress exposure. Psychopharmacology (Berl), 2008.

60. Lotrich, F.E. and B.G. Pollock, Meta-analysis of serotonin transporter polymorphisms and affective disorders. Psychiatr Genet, 2004. 14(3): p. 121-9.

61. Savelieva, K.V., S. Zhao, V.M. Pogorelov, I. Rajan, Q. Yang, E. Cullinan, and T.H. Lanthorn, Genetic disruption of both tryptophan hydroxylase genes dramatically reduces serotonin and affects behavior in models sensitive to antidepressants. PLoS ONE, 2008. 3(10): p. e3301.

62. Vitalis, T., O. Cases, S. Passemard, J. Callebert, and J.G. Parnavelas, Embryonic depletion of serotonin affects cortical development. Eur J Neurosci, 2007. 26(2): p. 331-44.

63. Fon, E.A., E.N. Pothos, B.-C. Sun, N. Killeen, D. Sulzer, and R.H. Edwards, Vesicular Transport Regulates Monoamine Storage and Release but Is Not Essential for Amphetamine Action. Neuron, 1997. 19(6): p. 1271-1283.

64. Wang, Y.M., et al., Knockout of the vesicular monoamine transporter 2 gene results in neonatal death and supersensitivity to cocaine and amphetamine. Neuron, 1997. 19(6): p. 1285-96.

65. Croft, B.G., G.D. Fortin, A.T. Corera, R.H. Edwards, A. Beaudet, L.-E. Trudeau, and E.A. Fon, Normal Biogenesis and Cycling of Empty Synaptic Vesicles in Dopamine Neurons of Vesicular Monoamine Transporter 2 Knockout Mice. Molecular Biology of the Cell, 2005. 16(1): p. 306-315.

66. Fukui, M., R.M. Rodriguiz, J. Zhou, S.X. Jiang, L.E. Phillips, M.G. Caron, and W.C. Wetsel, Vmat2 heterozygous mutant mice display a depressive-like phenotype. J Neurosci, 2007. 27(39): p. 10520-9.

67. Lira, A., et al., Altered depression-related behaviors and functional changes in the dorsal raphe nucleus of serotonin transporter-deficient mice. Biol Psychiatry, 2003. 54(10): p. 960-71.

68. Bengel, D., et al., Altered brain serotonin homeostasis and locomotor insensitivity to 3, 4-methylenedioxymethamphetamine ("Ecstasy") in serotonin transporterdeficient mice. Mol Pharmacol, 1998. 53(4): p. 649-55.

69. Mathews, T.A., D.E. Fedele, F.M. Coppelli, A.M. Avila, D.L. Murphy, and A.M. Andrews, Gene dose-dependent alterations in extraneuronal serotonin but not 
dopamine in mice with reduced serotonin transporter expression. J Neurosci Methods, 2004. 140(1-2): p. 169-81.

70. Montanez, S., W.A. Owens, G.G. Gould, D.L. Murphy, and L.C. Daws, Exaggerated effect of fluvoxamine in heterozygote serotonin transporter knockout mice. J Neurochem, 2003. 86(1): p. 210-9.

71. Kim, D.K., et al., Altered serotonin synthesis, turnover and dynamic regulation in multiple brain regions of mice lacking the serotonin transporter.

Neuropharmacology, 2005. 49(6): p. 798-810.

72. Li, Q., Cellular and molecular alterations in mice with deficient and reduced serotonin transporters. Mol Neurobiol, 2006. 34(1-2): p. 51-66.

73. Lebrand, C., et al., Transient uptake and storage of serotonin in developing thalamic neurons. Neuron, 1996. 17(5): p. 823-35.

74. D'Amato, R.J., M.E. Blue, B.L. Largent, D.R. Lynch, D.J. Ledbetter, M.E. Molliver, and S.H. Snyder, Ontogeny of the serotonergic projection to rat neocortex: transient expression of a dense innervation to primary sensory areas. Proc Natl Acad Sci U S A, 1987. 84(12): p. 4322-6.

75. Rebsam, A., I. Seif, and P. Gaspar, Refinement of thalamocortical arbors and emergence of barrel domains in the primary somatosensory cortex: a study of normal and monoamine oxidase a knock-out mice. J Neurosci, 2002. 22(19): p. 8541-52.

76. Holmes, A., D.L. Murphy, and J.N. Crawley, Abnormal behavioral phenotypes of serotonin transporter knockout mice: parallels with human anxiety and depression. Biol Psychiatry, 2003. 54(10): p. 953-9.

77. Holmes, A., Q. Lit, D.L. Murphy, E. Gold, and J.N. Crawley, Abnormal anxietyrelated behavior in serotonin transporter null mutant mice: the influence of genetic background. Genes Brain Behav, 2003. 2(6): p. 365-80.

78. Lucki, I., A. Dalvi, and A.J. Mayorga, Sensitivity to the effects of pharmacologically selective antidepressants in different strains of mice. Psychopharmacology (Berl), 2001. 155(3): p. 315-22.

79. Thakker, D.R., F. Natt, D. Husken, H. van der Putten, R. Maier, D. Hoyer, and J.F. Cryan, siRNA-mediated knockdown of the serotonin transporter in the adult mouse brain. Mol Psychiatry, 2005. 10(8): p. 782-9, 714. 
80. Maciag, D., K.L. Simpson, D. Coppinger, Y. Lu, Y. Wang, R.C. Lin, and I.A. Paul, Neonatal antidepressant exposure has lasting effects on behavior and serotonin circuitry. Neuropsychopharmacology, 2006. 31(1): p. 47-57.

81. Ansorge, M.S., M. Zhou, A. Lira, R. Hen, and J.A. Gingrich, Early-life blockade of the 5-HT transporter alters emotional behavior in adult mice. Science, 2004. 306(5697): p. 879-81.

82. Maciag, D., L. Williams, D. Coppinger, and I.A. Paul, Neonatal citalopram exposure produces lasting changes in behavior which are reversed by adult imipramine treatment. Eur J Pharmacol, 2006. 532(3): p. 265-9.

83. Hansen, H.H., C. Sanchez, and E. Meier, Neonatal administration of the selective serotonin reuptake inhibitor Lu 10-134-C increases forced swimming-induced immobility in adult rats: a putative animal model of depression? J Pharmacol Exp Ther, 1997. 283(3): p. 1333-41.

84. Hansen, H.H. and J.D. Mikkelsen, Long-term effects on serotonin transporter mRNA expression of chronic neonatal exposure to a serotonin reuptake inhibitor. Eur J Pharmacol, 1998. 352(2-3): p. 307-15.

85. Mirmiran, M., N.E. van de Poll, M.A. Corner, H.G. van Oyen, and H.L. Bour, Suppression of active sleep by chronic treatment with chlorimipramine during early postnatal development: effects upon adult sleep and behavior in the rat. Brain Res, 1981. 204(1): p. 129-46.

86. Ashburner, M. and C.M. Bergman, Drosophila melanogaster: a case study of a model genomic sequence and its consequences. Genome Res, 2005. 15(12): p. 1661-7.

87. Adams, M.D., et al., The Genome Sequence of Drosophila melanogaster. Science, 2000. 287(5461): p. 2185-2195.

88. Jordan, K.W., T.J. Morgan, and T.F. Mackay, Quantitative trait loci for locomotor behavior in Drosophila melanogaster. Genetics, 2006. 174(1): p. 271-84.

89. Foltenyi, K., R. Andretic, J.W. Newport, and R.J. Greenspan, Neurohormonal and neuromodulatory control of sleep in Drosophila. Cold Spring Harb Symp Quant Biol, 2007. 72: p. 565-71.

90. Wu, M.N., K. Koh, Z. Yue, W.J. Joiner, and A. Sehgal, A genetic screen for sleep and circadian mutants reveals mechanisms underlying regulation of sleep in Drosophila. Sleep, 2008. 31(4): p. 465-72. 
91. Seugnet, L., Y. Suzuki, L. Vine, L. Gottschalk, and P.J. Shaw, D1 receptor activation in the mushroom bodies rescues sleep-loss-induced learning impairments in Drosophila. Curr Biol, 2008. 18(15): p. 1110-7.

92. Neuser, K., T. Triphan, M. Mronz, B. Poeck, and R. Strauss, Analysis of a spatial orientation memory in Drosophila. Nature, 2008. 453(7199): p. 1244-7.

93. Baier, A., B. Wittek, and B. Brembs, Drosophila as a new model organism for the neurobiology of aggression? J Exp Biol, 2002. 205(Pt 9): p. 1233-40.

94. Liu, T., L. Dartevelle, C. Yuan, H. Wei, Y. Wang, J.F. Ferveur, and A. Guo, Increased dopamine level enhances male-male courtship in Drosophila. J Neurosci, 2008. 28(21): p. 5539-46.

95. Min, V.A. and B.G. Condron, An assay of behavioral plasticity in Drosophila larvae. J Neurosci Methods, 2005. 145(1-2): p. 63-72.

96. Hirsh, J., Time flies like an arrow. Fruit flies like crack? Pharmacogenomics J, 2001. 1(2): p. 97-100.

97. Kretzschmar, D., J. Tschape, A. Bettencourt Da Cruz, E. Asan, B. Poeck, R. Strauss, and G.O. Pflugfelder, Glial and neuronal expression of polyglutamine proteins induce behavioral changes and aggregate formation in Drosophila. Glia, 2005. 49(1): p. 59-72.

98. Dierick, H.A. and R.J. Greenspan, Serotonin and neuropeptide $F$ have opposite modulatory effects on fly aggression. Nat Genet, 2007. 39(5): p. 678-82.

99. Yuan, Q., W.J. Joiner, and A. Sehgal, A sleep-promoting role for the Drosophila serotonin receptor 1A. Curr Biol, 2006. 16(11): p. 1051-62.

100. Brand, A.H. and E.-L. Dormand, The GAL4 system as a tool for unravelling the mysteries of the Drosophila nervous system. Current Opinion in Neurobiology, 1995. 5(5): p. 572-578.

101. Bier, E., Drosophila, the golden bug, emerges as a tool for human genetics. Nat Rev Genet, 2005. 6(1): p. 9-23.

102. Saudou, F., U. Boschert, N. Amlaiky, J.L. Plassat, and R. Hen, A family of Drosophila serotonin receptors with distinct intracellular signalling properties and expression patterns. EMBO J, 1992. 11(1): p. 7-17.

103. Paxon, T.L., P.R. Powell, H.G. Lee, K.A. Han, and A.G. Ewing, Microcolumn separation of amine metabolites in the fruit fly. Anal Chem, 2005. 77(16): p. 5349-55. 
104. Chen, J. and B.G. Condron, Branch architecture of the fly larval abdominal serotonergic neurons. Dev Biol, 2008. 320(1): p. 30-8.

105. Valles, A.M. and K. White, Serotonin-containing neurons in Drosophila melanogaster: development and distribution. J Comp Neurol, 1988. 268(3): p. 414-28.

106. Lundell, M.J. and J. Hirsh, Temporal and spatial development of serotonin and dopamine neurons in the Drosophila CNS. Dev Biol, 1994. 165(2): p. 385-96.

107. Budnik, V. and K. White, Genetic dissection of dopamine and serotonin synthesis in the nervous system of Drosophila melanogaster. J Neurogenet, 1987. 4(6): p. 309-14.

108. Sykes, P.A. and B.G. Condron, Development and sensitivity to serotonin of Drosophila serotonergic varicosities in the central nervous system. Dev Biol, 2005. 286(1): p. 207-16.

109. Balaji, J., R. Desai, S.K. Kaushalya, M.J. Eaton, and S. Maiti, Quantitative measurement of serotonin synthesis and sequestration in individual live neuronal cells. J Neurochem, 2005. 95(5): p. 1217-26.

110. Johnson, R.D. and J.B. Justice, Model studies for brain dialysis. Brain Res Bull, 1983. 10(4): p. 567-71.

111. Clapp-Lilly, K.L., R.C. Roberts, L.K. Duffy, K.P. Irons, Y. Hu, and K.L. Drew, An ultrastructural analysis of tissue surrounding a microdialysis probe. J Neurosci Methods, 1999. 90(2): p. 129-42.

112. Hardie, S.L. and J. Hirsh, An improved method for the separation and detection of biogenic amines in adult Drosophila brain extracts by high performance liquid chromatography. Journal of Neuroscience Methods, 2006. 153(2): p. 243-249.

113. Dasari, S., K. Viele, A.C. Turner, and R.L. Cooper, Influence of PCPA and MDMA (ecstasy) on physiology, development and behavior in Drosophila melanogaster. Eur J Neurosci, 2007. 26(2): p. 424-38.

114. Stamford, J.A., In vivo voltammetry: promise and perspective. Brain Res, 1985. 357(2): p. 119-35.

115. Bruns, D. and R. Jahn, Real-time measurement of transmitter release from single synaptic vesicles. Nature, 1995. 377(6544): p. 62-65.

116. Bruns, D., D. Riedel, J. Klingauf, and R. Jahn, Quantal release of serotonin. Neuron, 2000. 28(1): p. 205-20. 
117. Robinson, D.L., B.J. Venton, M.L.A.V. Heien, and R.M. Wightman, Detecting Subsecond Dopamine Release with Fast-Scan Cyclic Voltammetry in Vivo. Clinical Chemistry, 2003. 49(10): p. 1763-1773.

118. Daws, L.C., G.M. Toney, G.A. Gerhardt, and A. Frazer, In vivo chronoamperometric measures of extracellular serotonin clearance in rat dorsal hippocampus: contribution of serotonin and norepinephrine transporters. J Pharmacol Exp Ther, 1998. 286(2): p. 967-76.

119. Daws, L.C., G.G. Gould, S.D. Teicher, G.A. Gerhardt, and A. Frazer, 5-HT(1B) receptor-mediated regulation of serotonin clearance in rat hippocampus in vivo. $\mathrm{J}$ Neurochem, 2000. 75(5): p. 2113-22. 


\title{
Chapter 2. Diffusion of small molecules into the larval Drosophila CNS
}

\begin{abstract}
The monoamine neurotransmitters serotonin and dopamine play key roles in human physiology and behavior. The complexity of these systems in mammals makes them difficult to study and Drosophila provides an attractive alternative. The small size of the fly CNS has been a barrier to the study of monoaminergic signaling and heretofore analysis was limited to measuring whole CNS levels of the neurotransmitters in homogenized tissue. Since signaling occurs through the rapid release of neurotransmitter into the extracellular space, prior techniques did not have the spatial or temporal resolution to measure it. This chapter describes the preliminary experiments in the optimization of fast scan cyclic voltammetry at inserted carbon fiber microelectrodes for use in the fly. Using the method developed, the diffusion of bath-applied dopamine into the isolated VNC is measured. A second method, fluorescence microscopy is used to measure the diffusion of another substance, sulforhodamine. This data is critical for future pharmacology experiments, which rely on drug diffusion, and paves the way for subsequent studies of more rapid changes in neurotransmitter levels, such as those that occur during signaling.
\end{abstract}

\section{Introduction}

The monoamine neurotransmitters serotonin and dopamine play key roles during development and in adult physiology and behavior [1-7]. Dysfunction in these neurotransmitter systems has been linked to disease states and drugs targeting them are the standard of care for many disorders [8-11]. The complexity monoaminergic signaling makes it difficult to study in mammals and Drosophila offers an attractive alternative due to its short life cycle, completed genomic sequence [12], and ease of 
molecular and genetic manipulation [13, 14]. Monoaminergic signaling is thought to occur via neurotransmitter diffusion through the extracellular space (ECS) $[15,16]$. The direct analysis of monoaminergic signaling therefore requires an approach that provides a high degree of spatial and temporal resolution. Such a technique was heretofore not available for Drosophila. This project was designed to optimize electrochemical monoamine detection for use in the $8 \mathrm{~nL}$ larval fly central nervous system (CNS) in order to expand the utility of this model organism.

Measurement of serotonin and dopamine concentrations in the fly has typically relied on whole-brain homogenization followed by analysis with high performance liquid chromatography (HPLC) [17] or capillary electrophoresis [18]. Both methods destroy the tissue utilized precluding repeat measurements in the same tissue. Furthermore, only total brain monoamine content can be quantified. Detection at inserted microelectrodes offers an attractive alternative. One such technique, Fast scan cyclic voltammetry (FSCV) has been well characterized for use in brain slices [19] and more recently for the detection of monoamine release from animals performing reward-mediated behaviors, such as cocaine seeking [20]. Electrodes continuously sample from the extracellular fluid, allowing direct measurement of the functional monoamine pool. The small size ( 7 $\mu \mathrm{m}$ in diameter) of the electrodes employed and high time resolution makes FSCV a good candidate technique for use in the fly.

This chapter delineates the initial experiments performed in the development of a method to perform real time monoamine measurements in the fly. The optimization of the recording buffer and dissection protocol are covered first. Diffusion of small molecules into the tissue is monitored to gain a better understanding of the time course of diffusion to the inserted electrode. This knowledge is critical for future pharmacology experiments, which rely on drug diffusing to the sampling area. Finally, implanted electrodes are used to monitor the diffusion of dopamine into the isolated CNS. The last 
set of experiments serves two purposes. First, it serves as a proof of principle to show that FSCV at an inserted microelectrode can be used to monitor changes in extracellular monoamine levels in the fly. Second, it provides additional information about the diffusion of small molecules into the CNS and how diffusion is affected by electrode placement or function of transporters in the tissue. This work paves the way for subsequent studies of endogenous monoamine release and clearance in the larval fly.

\section{Methods}

i. Fly stocks

The following fly stocks were obtained from Bloomington stock center (http://flystocks.bio.indiana.edu/): UAS-mCD8-GFP, Canton S (CS), ddc-GAL4. Flies containing Channelrhodopsin 2 (a gift from Christian Schroll, Universitat Wurzburg) were crossed to flies expressing Tph-GAL4 (a gift from Jaeson Kim, Korea Advanced Institute of Science and Technology) to generate a homozygous line.

\section{ii. Derivation of optimal recording buffer}

To test the effects of buffer on morphology, VNCs from 5 day old larvae were dissected in the buffer being tested then incubated for $60 \mathrm{~min}$ at room temperature prior to fixation in $4 \%$ paraformaldehyde for $1 \mathrm{~h}$ at room temperature and staining as described below. Four buffer compositions were evaluated for their effect on morphology; Phosphate buffered saline (PBS), HEPES-based buffer (124 mM NaCl, 3 mM KCl, 2mM $\mathrm{CaCl} 2 \cdot 7 \mathrm{H} 2 \mathrm{O}, 26 \mathrm{mM} \mathrm{NaHCO}, 1.25 \mathrm{mM} \mathrm{NaH} 2 \mathrm{PO} 4 \bullet \mathrm{H} 2 \mathrm{O}, 1 \mathrm{mM} \mathrm{MgSO} 4 \cdot 7 \mathrm{H} 2 \mathrm{O}, 20 \mathrm{mM}$ HEPES, 10 mM D-glucose), a modified schneiders buffer I termed CFE buffer (15.2 mM MgSO4, 21 mM KCl, 3.3 mM KH2PO4, 53 mM NaCl, 5.8 mM NaH2PO4, 5.4 mM CaCl2, 11.1 mM Glucose, $5.3 \mathrm{mM}$ Trehalose, $\mathrm{pH}=6.2$ ) and Ca-free CFE buffer. Schneiders buffer served as a positive control. Larvae from two strains were used for these 
experiments ddc-GAL4 ;UAS-mCD8-GFP and Tph-GAL4; UAS-ChR2. The former were stained for both serotonin and GFP, the later for serotonin only. For staining, samples were incubated in Phosphate buffered saline with 1\% tween (PBT) overnight at room temperature with primary antibodies, 1:1000 anti-serotonin (rabbit polyclonal, ImmunoStar) and/or 1:1000 anti-GFP (chicken polyclonal, Abcam). Alexafluor goat antirabbit and/or goat anti-chicken (Molecular Probes) were used at 1:1000 in PBT overnight at room temperature. Samples were mounted to slides in $90 \%$ glycerol/2.5\% $1,4-$ diazabicyclo[2.2.2]octane and allowed to clear overnight prior to examination under a confocal microscope. Changes in the morphology of serotonergic and/or dopaminergic neurons were identified by comparing samples incubated in test buffers to control samples as well as those in the Condron laboratory database.

\section{iii. Diffusion of rhodamine into the isolated VNC}

Brains from 5 day old larvae were dissected in Schneiders buffer and the optic lobes were removed fully isolate the VNC and create a cut edge. Isolated VNCs were then placed in a 40ul drop of media onto the surface of a \#1 coverslip so that the neuropil is closest to the glass surface. The coverslip was then placed, sample-side down, onto a well created by two coverslips on top of a glass slide. After positioning on the confocal microscope, 1:100 sulforhodamine (SR101) was added and a stack of 10 images through the neuropil was obtained every $2 \mathrm{~min}$. Images were imported into imageJ and 3 bins of $\sim 20 \mu \mathrm{m} \times 20 \mu \mathrm{m}$ were used to measure mean gray levels. Overall changes in gray levels were obtained for each sample by averaging 3 bins from 2 slices of neuropil. Mean grey levels were subsequently plotted using excel software. 


\section{iv. Instrumentation and electrochemistry}

Carbon-fiber microelectrodes were manufactured from single T-650 carbon fibers, $7 \mu \mathrm{m}$ in diameter (Cytec Engineering Materials, West Patterson, NJ), as previously described (B. J. Venton et al., 2006). A Dagan Chem Clamp potentiostat was used to collect electrochemistry data (Dagan, Minneapolis, MN; custom-modified). Data acquisition software and hardware were the same as described by Heien et al (M. L. Heien et al., 2003). The electrode potential was continuously scanned from -0.4 to $1.0 \mathrm{~V}$ and back at $400 \mathrm{~V} / \mathrm{s}$ every $100 \mathrm{~ms}$, even when data was not being collected. A silversilver chloride wire was used as a reference electrode. Electrodes were calibrated with 1 $\mu \mathrm{M}$ dopamine prior to and after use in situ. Electrochemical data was analyzed using Tar Heel CV software (M. L. Heien et al., 2004). Background subtraction for cyclic voltammograms was accomplished by using 10 scans collected 1 minute before addition of dopamine.

\section{v. Preparation of ventral nerve cords (VNCs) for dopamine diffusion assays}

Brains from 5 day old larvae were dissected in CFE buffer. The optic lobes were removed by means of a horizontal cut in the anterior-most portion of the VNC, which was then placed, neuropil side down, onto the bottom of a petri plate in $3 \mathrm{ml}$ fresh buffer, as illustrated in figure 1. An electrode was implanted using a micromanipulator, while the sample was visualized under a 40X water immersion objective. The manipulator was angled so as to place the active potion of the electrode within the middle portion of the neuropil when the electrode was inserted to a distance of 4-6 segments away from the cut edge. Each CNS was allowed to settle for $5 \mathrm{~min}$ after electrode implantation prior to onset of experiments, to allow the electrode background current to stabilize. 

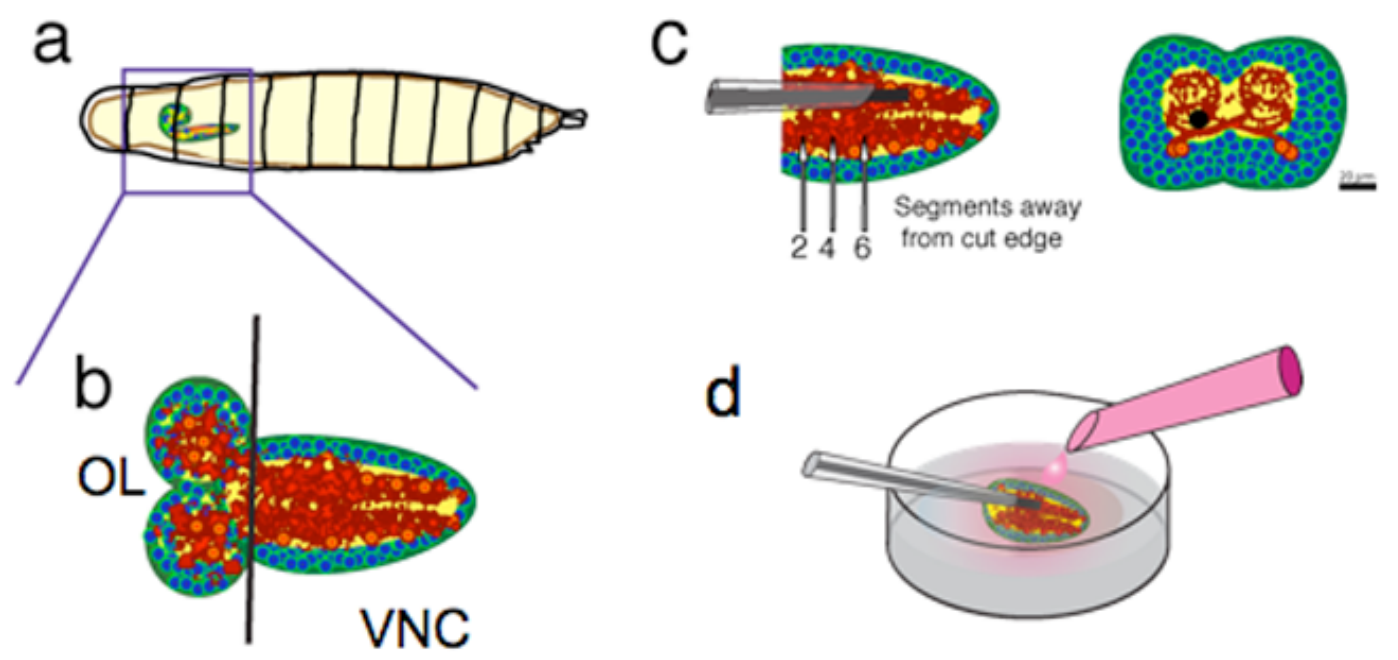

Figure 1: Preparation of samples for detection of dopamine diffusion. (a) 5 day old larvae is dissected to isolate the central nervous system. (b) The optic lobes (OL) are removed from the ventral nerve cord (VNC) via a vertical cut. (c) An electrode is inserted through the cut edge into the position shown on this to-scale diagram of the isolated VNC. (d) A diagram showing the isolated VNC in a bath of buffer within a petri plate. Dopamine (pink) is added to the bath with a pipette.

\section{vi. Diffusion of dopamine into the isolated VNC}

To monitor the diffusion of dopamine into the isolated VNC, a carbon-fiber microelectrode was inserted 4-6 segments into an isolated VNC. After 4 minutes of baseline data were collected, 20,40 , or $80 \mathrm{uM}$ dopamine in $1 \mathrm{ml}$ fresh media was added to the bath buffer, bringing the final dopamine concentration to 5,10 , or $20 \mathrm{uM}$ dopamine respectively. FSCV data was obtained in a series of 4 minute long, continuous files. Signal CVs were compared to those obtained during electrode calibration with $10 \mu \mathrm{M}$ dopamine to confirm the identity of the signal observed in situ. Current was measured at the calibrated oxidation potential of dopamine and this data was exported into Graph Pad Prism to monitor dopamine diffusion. Once files were joined to make a trace, the inflection point was determined and a time to inflection point for each trace was 
calculated. The height of the peak and time to inflection were the primary measures obtained in this assay.

To test the effect of electrode position on the diffusion of dopamine, the electrode was inserted into different regions of the neuropil and $10 \mu \mathrm{M}$ dopamine was added to the bath. To test the effect of rest time, dissected VNCs were challenged with $10 \mu \mathrm{M}$ dopamine either immediately after dissection or after a $30 \mathrm{~min}$ resting period. To test the effect of dopamine transporter (DAT) function on dopamine diffusion, the time to inflection point was measured after adding 5,10 , or $20 \mu \mathrm{M}$ dopamine to VNCs with DAT function inhibited by genetic (DAT hypomorph larvae) or pharmacologic (Incubation in $10 \mu \mathrm{M}$ Nomifensine) means. All VNCs utilized for pharmacology and genetics experiments were given 30 minutes to rest prior to the initiation of experiments. When nomifensine was used, it was present in all solutions, including initial dissection buffer and calibration buffers. Any drug effects on dopamine detection were therefore accounted for in the calibration step. Pooled data was analysis, including student's ttests, was conducted using Graph Pad prism and excel software.

\section{Results}

\section{i. Derivation of optimal recording buffer}

Schneider's buffer is routinely used for fly CNS culture but initial experiments showed that exposure to this buffer rapidly fouled the electrodes (data not shown). The Schneiders buffer reagent obtained from Sigma was found to contain several electroactive substances, such as formic acid, which are likely responsible for the fouling effect of Schneiders buffer. Therefore, four simpler buffer compositions were tested; PBS, HEPES-based buffer typically utilized by the Wightman laboratory for mammalian brain slice experiments, a modified Schneiders buffer containing only the salts and sugars (this formulation we have termed CFE buffer), and Ca-free CFE buffer. 
Schneiders buffer served as a positive control for the experiment. All simpler buffer compositions were first evaluated for electrode fouling effects by exposing the electrodes to the buffer for a prolonged time $(1+$ hours $)$ then testing the electrode with a standard concentration of monoamine. Electrodes exposed to all 4 candidate buffers showed minimal signs of fouling.

Candidate buffers were tested for their ability to maintain healthy fly CNS tissue. Changes in the morphology of serotonergic and/or dopaminergic neurons, as viewed by confocal microscopy provide a sensitive assay for tissue damage [21]. Larval VNCs in were dissected in each buffer, allowed to incubate for 1 hour, then fixed and stained for serotonin and/or GFP. Two fly strains were used. The first was homozygous for ddcGAL4 ;UAS-mCD8-GFP, which expresses GFP in serotonergic and dopaminergic neurons and is routinely used by the B. Condron laboratory for histological assays. The second was homozygous for Tph-GAL4; UAS-ChR2, and was to be used for subsequent FSCV experiments in Ch 2 of this thesis. The results from this experiment are shown in figure 2. The data is organized into three columns based on the type of larvae and the power at which it was imaged. VNCs from Tph-GAL4; UAS-ChR2 larvae stained for serotonin and imaged at 50x are shown on the left. VNCs from ddc-GAL4; UAS-mCD8GFP larvae stained for serotonin and GFP and imaged at 50x or 100x are shown in center or right hand column respectively.

VNCs dissected in Schneiders buffer (a-c) show healthy tissue morphology. Serotonergic neurons, most clearly visible in a VNC stained for serotonin only (a), show a prominent network of inter-connected projections within the larval neuropil. Axonal swellings, known as varicosities, are connected by fine axonal branches. Some minor damage is visible as disrupted connections between varicosities within 1-2 segments of the cut edge but the majority of the tissue shows healthy morphology. VNCs dissected in 
PBS (d-f) show the most severe tissue damage. This damage is especially obvious in d, which shows a stack of confocal images taken through the middle of the neuropil.

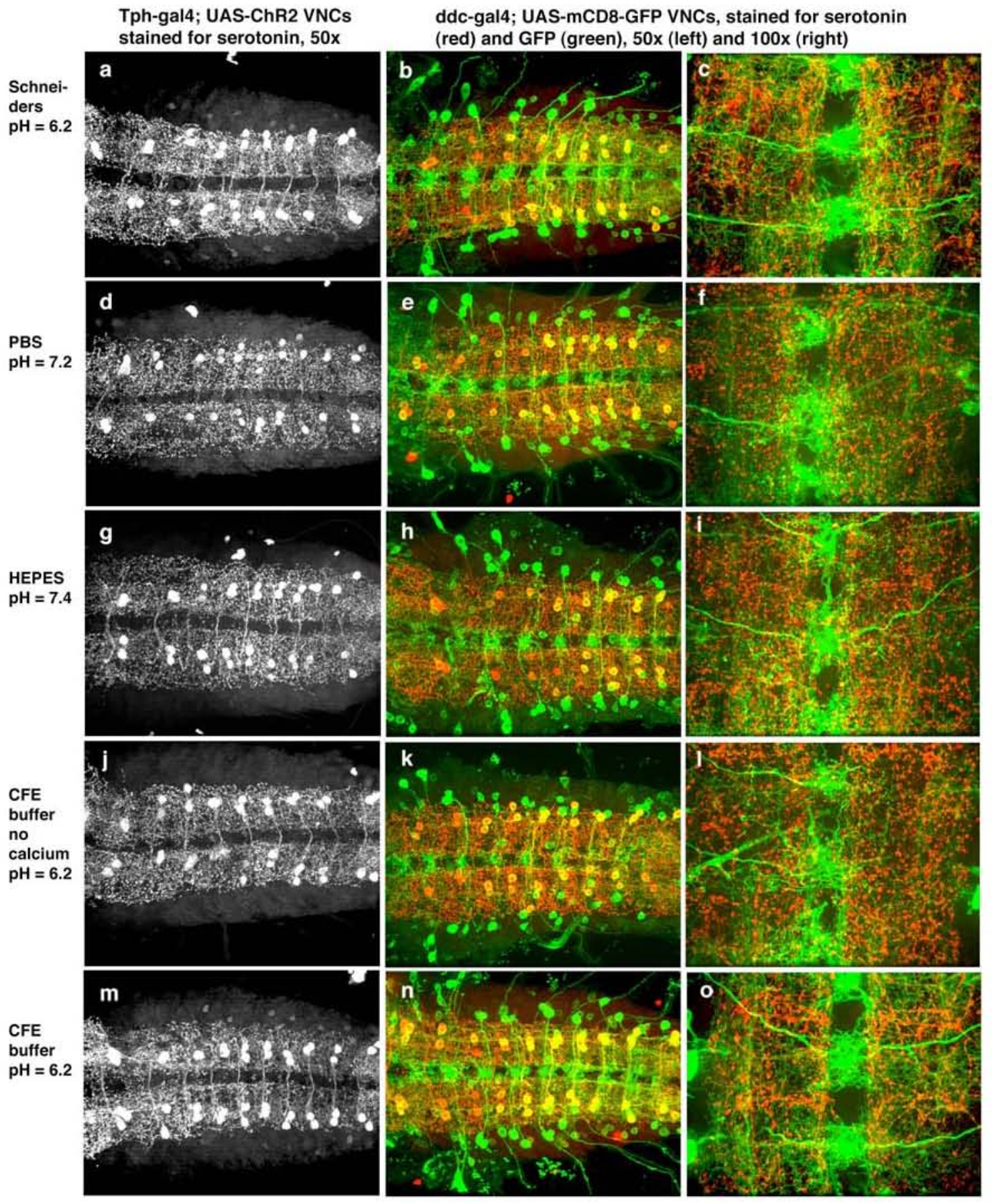

Figure 2: Effect of buffer composition on the morphology of monoaminergic neurons. 5 day old VNCs were dissected in buffer of composition specified below, cultured for $1 \mathrm{~h}$ then fixed and stained for serotonin and/or GFP. (left column) VNCs from Tph-gal4; 
UAS-ChR2 larvae, which express channelrhodopsin2 in serotonergic neurons. VNCs were stained for serotonin and imaged with a 50x objective on a confocal microscope. Each image represents a stack of $\sim 250$ slices. Cut edge is $\sim 1$ segment to the left of the left-most edge of the image. (center column) VNCs from ddc-gal4; UAS-mCD8:GFP larvae, which express GFP in serotonergic and dopaminergic neurons and are routinely used for histology in the B. Condron laboratory. Samples were stained for serotonin (red) and GFP (green) and imaged in the same way as samples from left column. (right column) VNCs from ddc-gal4; UAS-mCD8:GFP larvae prepared in same was as center column and imaged using a 100x oil immersion objective, with each image representing a composite stack of $\sim 200$ slices through the middle of the neuropil. These VNCs were imaged at 4-6 segments away from cut edge. (a-c) Schneiders buffer, $\mathrm{pH}=6.2,(\mathrm{~d}-\mathrm{f})$ PBS, $\mathrm{pH}=7.2$, (g-i) HEPES buffer, $\mathrm{pH}=7.4$, (j-I) CFE buffer without calcium, $\mathrm{pH}=6.2$. The term CFE buffer denotes the modified Schneiders buffer I optimized for use with FSCV, (m-o) CFE buffer, $\mathrm{pH}=6$.2. Note evidence of tissue degeneration, including varicosity swelling evident as red spheres as opposed to fibers and destruction of fine axonal branches and fibers throughout the VNC in all but the bottom and top-most rows (a-b, m-o) of samples. Like samples incubated in Schneiders buffer, those incubated in CFE buffer show minimal damage, primarily localized to 2-3 segments away from the cut edge.

The normally fibrous dopaminergic projections (green) are fragmented and have a hazy, ragged appearance. Serotonergic projections (red) are also highly fragmented and swollen thereby making them appear like isolated red spheres as opposed to interconnected thickenings and branches. Similar axonal fragmenting throughout the VNC is evident in samples dissected in HEPES buffer as well as calcium-free CFE buffer, although not as severe.

Much of the damage likely results from the swelling of axonal structures due to the buffer being too hypotonic. A working osmolar level between $150-200 \mathrm{mOsm}$ is critical to maintaining the health of Drosophila tissue (Schneiders packet) and the osmolarity of PBS is significantly below this level. CFE buffer, with an osmolarity of $\sim 190 \mathrm{mOsm}$, is in the optimal range. HEPES-based buffer with an osmolarity of $\sim 350$ also produces 
observable tissue damage as does the failure to include calcium in the buffer. Incubation in CFE buffer maintains the health of the neuropil. Tissue incubated in CFE buffer $(j-I)$ is indistinguishable from that incubated in Schneiders (a-c). Fine axonal structures are intact and serotonergic projections are interconnected and normal with respect to size and shape. Based on this set of immunohistochemistry experiments, CFE buffer was chosen for use in all subsequent experiments in this thesis.

\section{ii. Diffusion of SR101 into the isolated VNC}

To monitor the diffusion of sulforhodamine (SR101), isolated VNC were mounted onto a coverslip as shown in a diagram in Fig 3a and SR101 was added to the bath buffer while monitoring the diffusion of the molecule by confocal microscopy. SR101 fluoresce was visualized in the $568 \mathrm{~nm}$ channel and increased intensity correlated with the diffusion of the molecule into the area. The time to reach $1 / 2$ maximal fluorescence ranged from 10 to 30 minutes at a distance of 4-6 segments away from the cut edge. As can be seen in a representative sample (Fig 3b), diffusion proceeded primarily through the cut edge with points closer to the edge exhibiting faster increases in signal.

Significant exclusion of SR101 by the intact glial sheath was evident. Even after 2 hours, much of the VNC remained free of SR101 fluorescence. Occasional samples also showed diffusion from a fixed point in the VNC, likely coinciding with a small tear in the glial sheath.

SR101 signal was quantified with imageJ software by measuring mean gray levels within three $\sim 20 \mu \mathrm{m} \times 20 \mu \mathrm{m}$ bins. The bins were placed in random locations within the neuropil region 3-5 segments away from the cut edge. No observable differences in the time course of SR101 diffusion were noted between bins in this area. The increase of signal at bins placed 2-3 segments away from the cut edge occurred faster than those placed 6-7 segments away (data not shown). This experiment shows 
that even molecules that are largely excluded by the intact glial sheath can diffuse up to 6 segments away from the cut edge. The majority of diffusion occurred within 40 minutes the addition of SR101 to the buffer and neuropil more than 8 segments away from the cut edge did not experience increased fluoresecence even after $2 \mathrm{~h}$. This suggests that small molecule diffusion into the VNC may be retarded after prolonged incubation.

\section{a, b. Diffusion of rhodamine into isolated nerve cord}
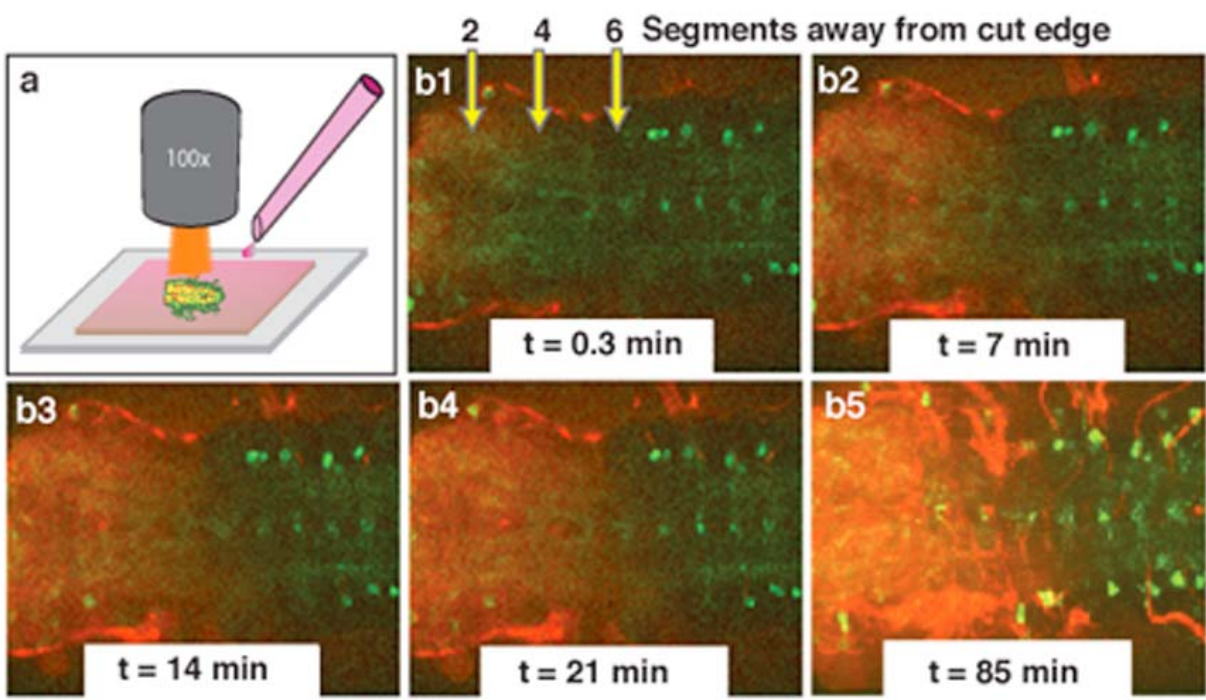

\section{c. Pooled data of diffusion of rhodamine into isolated nerve cord}

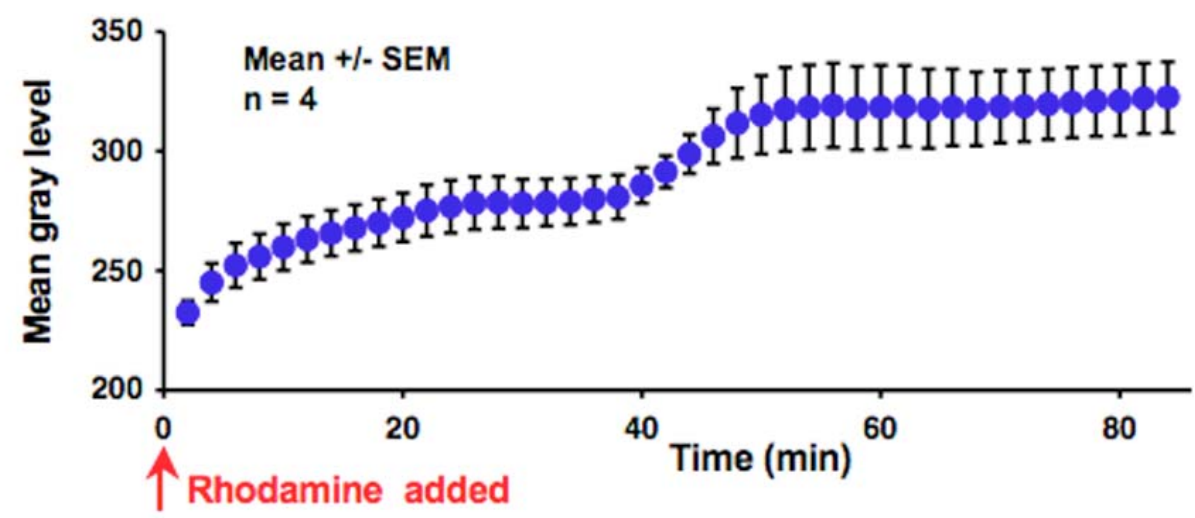

Figure 3: Diffusion of SR101 into the isolated VNC. 5 day old VNCs from ddc-gal4; UAS-mCD8:GFP larvae were dissected and imaged on a confocal microscope to 
visually monitor the diffusion of rhodamine into the VNC. A series of 30 slices through the middle of the neuropil was chosen. (a) Schematic of assay, showing isolated VNC within a dual-coverslip chamber to which SR101 was added. (b1-b5) Images from representative VNC imaged at 40x showing the diffusion of bath-applied SR101 to a distance of 4-6 segments away from the cut edge. Small fluorescent molecules such as SR101 are excluded from the nerve cord by an intact glial sheath, as evidenced by the absence of SR101 (red) 8-10 segments away from the cut edge many minutes after significant diffusion has occurred closer to the cut edge. (c) Pooled data from 4 samples showing the quantified increase in fluorescence within the neuropil over time. Note an initial rapid increase in signal followed by a secondary increase around 40 minutes. The time to the second inflection point for SR101 diffusion is longer than for dopamine and may reflect exclusion of this molecule by the glial sheath.

\section{iii. Diffusion of dopamine into the isolated VNC}

To monitor the diffusion of dopamine into the isolated VNC, a carbon fiber microelectrode, $7 \mu \mathrm{M}$ in diameter and $50 \mu \mathrm{M}$ in length, was inserted into position to sample from the neuropil 4 to 6 segments away from the cut edge. A diagram (Fig 1) shows a schematic of dissection and electrode implantation. The dissection protocol was based on that utilized for immunohistochemistry by the B. Condron laboratory. A standard, triangular shaped waveform, from -0.4 to $1.0 \mathrm{~V}$ and back at $400 \mathrm{~V} / \mathrm{s}$ every $100 \mathrm{~ms}$, was continually applied to the electrode. The current output at a potential corresponding to the calibrated oxidation potential of dopamine was plotted against time. Current measurements were converted to concentrations using electrode postcalibration with a standard. Cyclic voltammograms, plots of current vs. voltage, provided a means for identifying the species detected. The shape of the CV results from a combination of large background charging currents and smaller faradaic currents resulting from electron transfer to the analyte. Due to the temporal stability of the charging current, the background could be subtracted to yield an analyte-specific CV. 
Figure 4. An inserted electrode is used to monitor the diffusion of dopamine into the

a. Diffusion of dopamine into isolated nerve cord

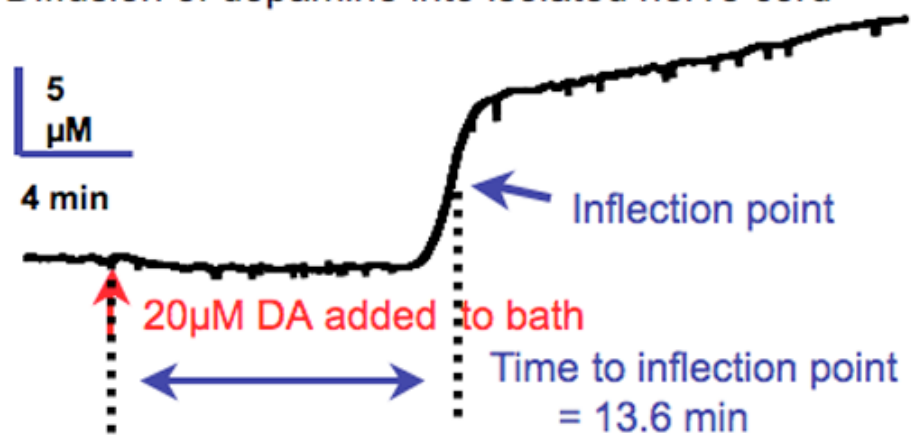

b. Detection of dopamine in isolated nerve cord

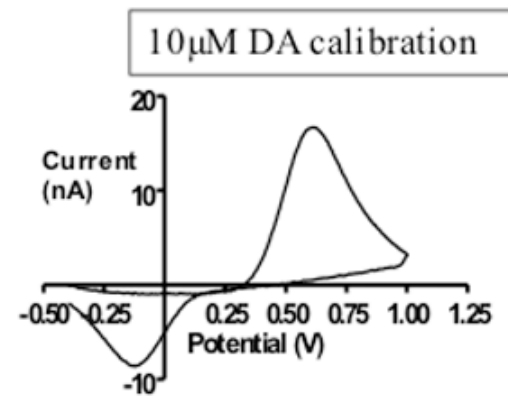

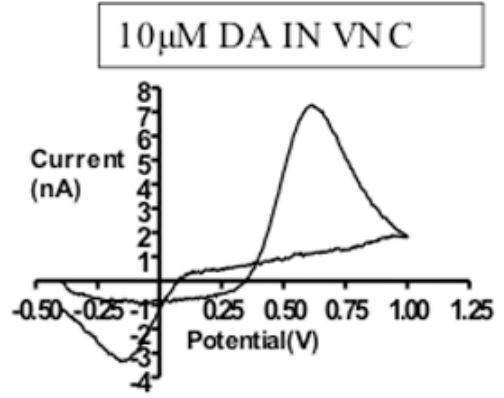
isolated VNC. (a) After the collection of 250 s of baseline, $20 \mu \mathrm{M}$ dopamine is added to the bath. Current at the calibrated oxidation potential of dopamine is constantly recorded and the output is modeled as a sigmoidal curve with an inflection point, as marked on the plot. The inflection point is the point at which the current is at a half-maximal value. (b) Cyclic voltammograms of signal during calibration

with dopamine in buffer as compared to CVs from signal in VNC. Note the identical location of peaks, showing that dopamine was detected in the isolated VNC.

The inserted electrode detected dopamine that had diffused into the neuropil. A trace of signal taken at the calibrated oxidation potential of dopamine from a representative sample (Fig 4a) showed an increase of current due to dopamine beginning 12 minutes after addition of dopamine to the bath buffer. As with diffusion measured via increase in fluorescence signal, a time to inflection point was calculated; the point utilized for the measure is shown on the trace. A background-subtracted CV from another representative sample (Fig $4 b$ ) shows that the signal CV was identical in shape but smaller in amplitude to that obtained during electrode calibration with $10 \mu \mathrm{M}$ dopamine, confirming that the increase in observed signal at the oxidation potential of 
dopamine was due to dopamine diffusing into the neuropil. Signal size in the sample was smaller than that applied to the bath.

Figure 5: Position of electrode does not affect the time till dopamine diffuses into the

a. Time to inflection point vs. medio-lateral position of electrode.

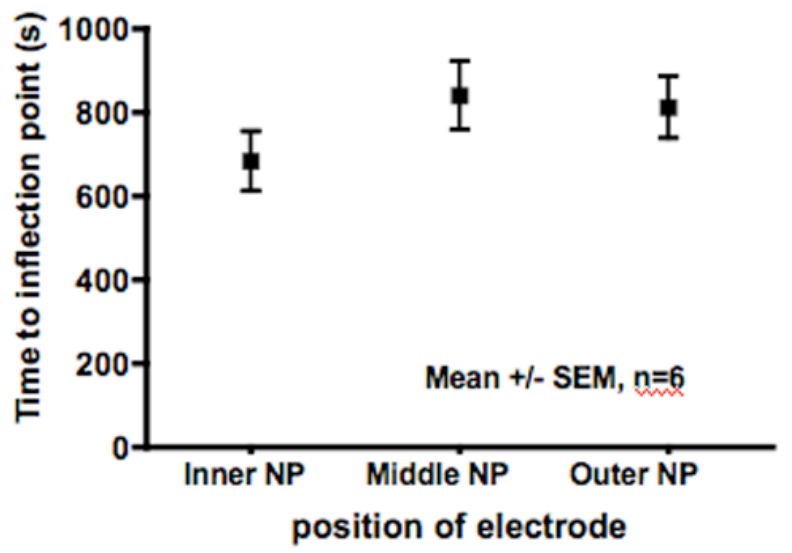

b. Time to inflection point vs. electrode distance from cut edge.

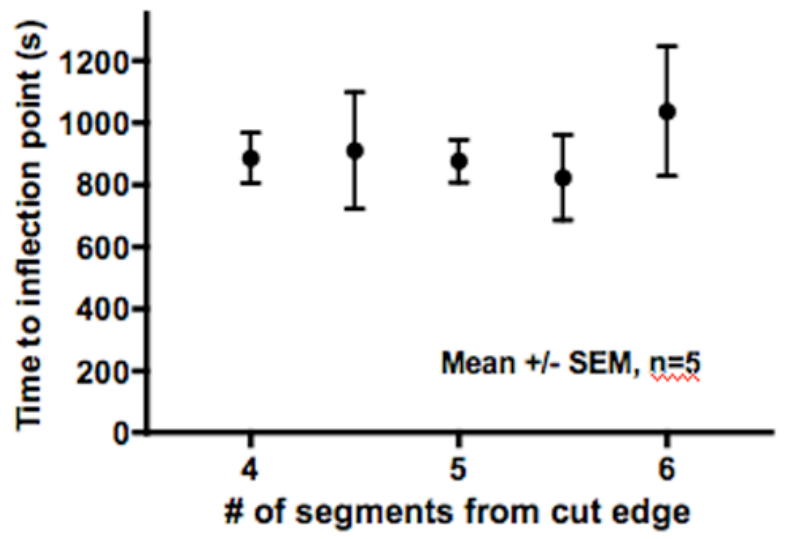

area. (a) Time to inflection point vs. position of electrode in the neuropil. No significant differences based on electrode position were observed. (b) Time to inflection point vs. distance from cut edge. Distance is determined visually as the approximate number of segments from the cut edge till the outer-most edge of the electrode. This 2 segment window was chosen based on fluorescent dye diffusion data showing that dye diffuses in approximately 6 segments. When the electrode is placed less than 4 segments away, the incidence of aberrant runs due to communication between the electrode and the bathing buffer increased. To avoid aberrant

runs and ensure that the electrode did not encounter tissue damaged during dissection, the electrode was placed no less than 4 segments away.

The effect of position within the neuropil on the diffusion of dopamine to the electrode was tested. The electrode was inserted into different regions of the neuropil and the time to the signal inflection point after the addition of $10 \mu \mathrm{M}$ dopamine to the bath 
was calculated (Fig 5). Within the window of 4 to 6 segments away from the cut edge, the medio-lateral position (Fig 5a) and distance from the cut edge (Fig 5b) did not significantly change the time to inflection point, indicating that dopamine gets to this window within 20 minutes. This is consistent with the data obtained from the visual monitoring of the diffusion of rhodamine into the VNC, where increased fluorescence was seen across the entirety of the neuropil up to 6 segments away from the cut edge. When the electrode was brought closer to the cut edge, the number of aberrant runs increased. Runs were counted as aberrant when electrode signal increased within 1 minute of adding dopamine to the bath. Aberrant runs likely resulted from tissue damage creating a direct channel between the electrode and the buffer.

Figure 6: Effect of waiting 30 minutes prior to the initiation of experiments. A trend

Time to inflection point vs. applied dopamine concentration

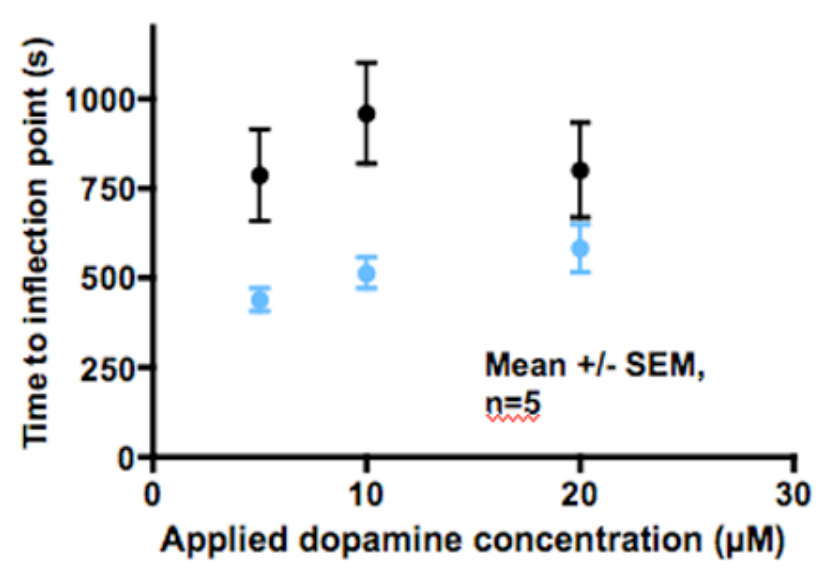

towards increased time to inflection is observed when - no wait - 30 min wait samples are allowed to incubate in buffer for 30 minutes prior to the addition of dopamine to the bath. Observed differences were not significant.

The effect of wait time on dopamine diffusion was tested by incubating VNCs for 6 or 30 minutes after dissection prior to the insertion of the electrode and onset of experiments. As with prior experiments, the main measure was time to inflection point. Pooled data from 5 samples (Fig 6) shows a trend towards less rapid entry of dopamine into the VNC when dopamine was added to the bath after a 30 minute incubation. 
Although not significant for this number of samples, the trend is consistent with that observed using SR101 fluorescence. During the 30 minute rest time, the properties of the cut edge may change thereby retarding small molecule diffusion.

Figure 7: Diffusion of dopamine into the isolated VNC. (a) Pooled data, mean +/- SEM,

a. Time to inflection Point vs applied dopamine concentration
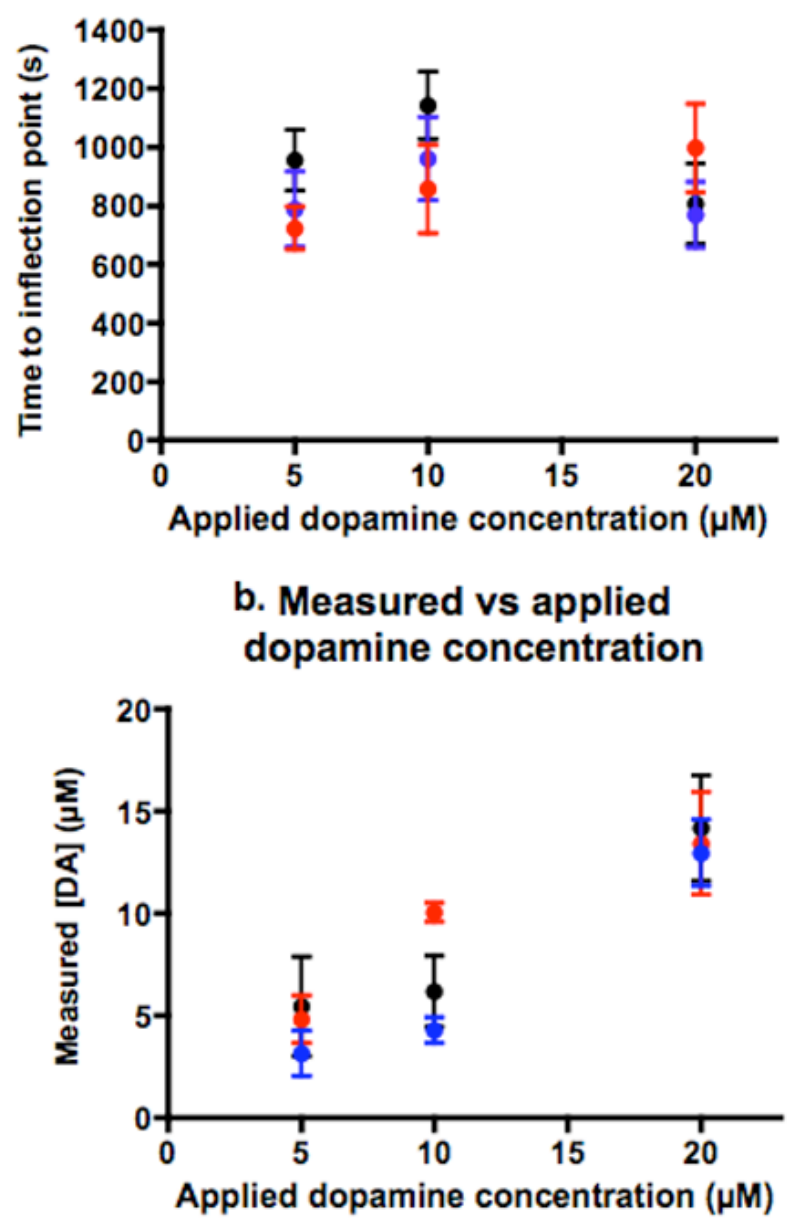

$\mathrm{n}=(4-7)$ showing effect of applied dopamine concentration and

- WT

- DATnull

- Nomifensine

- WT

- DATnull

- Nomifensine transporter efficacy on time to inflection point. No significant differences between the groups were measured, all groups showed $1 / 2$ maximal signal at approximately 15 minutes. (b) Pooled data showing measured vs. applied concentrations.

Although no significant differences were observed in groups differing in dopamine transporter function, increasing applied

concentrations produced a linear increase in measured dopamine. Measured dopamine concentrations tended to be lower than applied.

Dopamine is removed from the extracellular space by the actions of the dopamine transporter (DAT). To test the effect of transporter function on diffusion to the 
electrode, the time to inflection point was measured after adding 5 , 10 , or $20 \mu \mathrm{M}$ dopamine to VNCs with DAT function inhibited by genetic (DAT hypomorph larvae) or

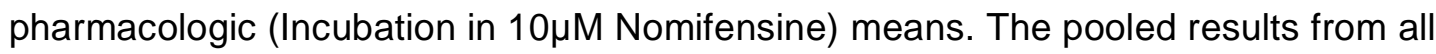
groups (Fig 7) show that dopamine diffuses to the electrode within 20 minutes regardless of DAT function. No significant differences in mean time to inflection point were observed. The concentration detected in the VNCs was proportional but slightly lower than that added to the bath (Fig 7b). This may be due to incomplete tissue permeation by dopamine or errors due to shifting baseline.

\section{Discussion}

The small size of the larval fly CNS has posed a considerable challenge to the measurement of monoamine signaling. Prior techniques typically employed pooled tissue homogenate and did not offer the temporal or special resolution necessary to quantify monoamine signaling. This work has focused on adapting FSCV at carbon microelectrodes for use in larval Drosophila. Because the microelectrodes sampled from the ECS, they provided for direct measurement of the functional monoamine pool. This work allowed, for the first time, the real-time detection of changes in extracellular monoamine levels in the fly larva.

Contraction of cardiac and skeletal muscles prevents the immobilization of the CNS in the intact fly larvae. A whole-CNS culture system allows for detailed morphological measurements to be performed within the intact, isolated larval VNC [22]. Comparable to mammalian slice preps, VNC culture offers the advantage that the majority of inter-neuronal connections are undamaged and in an intact CNS. Several aspects of this system, including dissection protocol, prep for electrode insertion, and buffer composition were modified to enable FSCV-mediated monoamine detection. Schneider's buffer is ideal for longer-term (6-8 hours) culture but fouls the 
microelectrodes. Simpler buffer compositions were tested in order to reach a compromise between the limits imposed by FSCV and the ionic and small molecule concentrations necessary to preserve good tissue integrity for the length of a typical experiment. The optimized buffer, termed CFE buffer, was based on the salt and sugars in Schneider's buffer, did not foul the electrodes and maintained the health of CNS neuronal tissue. This buffer was used for all subsequent FSCV experiments.

For future pharmacology experiments, it is essential to ensure that small molecules can diffuse acutely to the site of electrode implantation. Fluorescence microscopy allowed visual evaluation of small molecule diffusion into the isolated VNC. SR101 is charged and consequently heavily excluded by the glial sheath, which serves as a blood brain barrier in Drosophila [23, 24]. The time course for SR101 diffusion therefore represented the worst-case scenario for small molecule diffusion into the VNC. Since the majority of the glial sheath remains intact in cultured VNCs most SR101 diffusion should occur through the cut edge at the anterior-most portion of the VNC. As expected, SR101 diffused through the cut edge to a distance of 6 segments within 20-30 minutes. Diffusion did not ensure complete penetration of the VNC by SR101, however and neuropil greater than 8 segments away from the cut edge remained free of SR101 even after more than 2 hours. Because glial cells can move rapidly to seal off defects [25], it is possible that continuing SR101 diffusion through the cut edge was interrupted by a reforming glial sheath. This data guided the development of electrode insertion procedures for FSCV experiments. To ensure that even molecules heavily excluded by the glial sheath would reach the electrode while avoiding potentially damaged areas close to the cut edge, electrodes were inserted to a distance of 4-6 segments away from the cut edge.

As a proof of principle, microelectrodes were used to monitor the diffusion of bathapplied dopamine into the isolated VNC. Dopamine is easily detected and does not 
significantly adsorb onto the electrode, making it well suited for larger and longer-term exposures. Given enough time, bath applied dopamine should diffuse to the electrode several segments away from the cut edge. Approximately 12 minutes after applying dopamine to the bath solution, an increase in signal was observed at the electrode. The signal and calibration CVs were very similar, consistent with the detection of dopamine. Therefore, dopamine can diffuse into the VNC and monoamine detection in Drosophila is possible.

To explore the role of transport in limiting diffusion of dopamine to the electrode, DAT function was inhibited either pharmacologically by adding nomifensine or genetically by employing DAT hypomorph animals. At lower concentrations of bathadded dopamine, the dopamine transporter, which has an affinity for dopamine of 2.9 $\mu \mathrm{M}$ [26] should act to clear dopamine from the extracellular space, thereby retarding its diffusion to the implanted electrode. There were no significant differences in time to inflection point between VNCs lacking and those with normal DAT function. This likely occurred because high bath dopamine concentrations rapidly overwhelmed DAT function. Smaller concentrations gave unreliable increases in signal and therefore I was not able to employ concentrations low enough to measure changes due to uptake.

The high variability in time to and detection of signal increases likely resulted from baseline changes occurring throughout the experiment. Because the acquisition of measurements relies on background subtraction, FSCV is best suited for the measurement of rapid changes in extracellular monoamine levels. Although the background remains relatively stable on a time scale of 50-100 seconds [27], drift does occur across voltages and introduces progressively larger errors as the length of measurements increases. Monitoring dopamine diffusing from the buffer into the neuropil was therefore challenging because it occurred on a timescale of tens of minutes. The majority of baseline drift occurring in these experiments proceeded in the downward 
direction, therefore observed increases in signal likely underestimate the actual signal increase. This is evident when comparing observed dopamine concentration to that added to the bath. Observed concentrations were often $4-5 \mu \mathrm{M}$ lower than bath concentrations. The development of more rapid induction of monoamine release will decrease errors due to baseline drift and make better use of the subsecond temporal resolution of FSCV.

The work presented in this chapter has been instrumental to optimizing FSCV for the detection of changes in extracellular monoamine concentrations in the fruit fly. An optimal buffer composition, dissection, and electrode insertion protocols were developed. FSCV at an implanted microelectrode was used to monitor the diffusion of dopamine to the site of insertion and show that the molecule diffuses to a distance of 4-6 segments away from the cut edge within 20 minutes. Fluorescence microscopy was used to monitor the diffusion of another small molecule, SR101, into the isolated VNC. The results from this experiment showed that even molecules that are typically excluded by the glial sheath can diffuse to the area occupied by the inserted electrode within 2030 minutes. This knowledge is critical to future pharmacology experiments, which rely on drug diffusion. 


\section{References:}

1. Barnes, N.M. and T. Sharp, A review of central 5-HT receptors and their function. Neuropharmacology, 1999. 38(8): p. 1083-152.

2. Boulougouris, V. and E. Tsaltas, Serotonergic and dopaminergic modulation of attentional processes. Prog Brain Res, 2008. 172: p. 517-42.

3. Gaspar, P., O. Cases, and L. Maroteaux, The developmental role of serotonin: news from mouse molecular genetics. Nat Rev Neurosci, 2003. 4(12): p. 100212.

4. Gonzalez-Burgos, I. and A. Feria-Velasco, Serotonin/dopamine interaction in memory formation. Prog Brain Res, 2008. 172: p. 603-23.

5. Hunt, P.N., J. Gust, A.K. McCabe, and M.M. Bosma, Primary role of the serotonergic midline system in synchronized spontaneous activity during development of the embryonic mouse hindbrain. J Neurobiol, 2006. 66(11): p. 1239-52.

6. Jacobs, B.L. and E.C. Azmitia, Structure and function of the brain serotonin system. Physiol Rev, 1992. 72(1): p. 165-229.

7. Vitalis, T. and J.G. Parnavelas, The role of serotonin in early cortical development. Dev Neurosci, 2003. 25(2-4): p. 245-56.

8. Borah, A. and K.P. Mohanakumar, Long-term L-DOPA treatment causes indiscriminate increase in dopamine levels at the cost of serotonin synthesis in discrete brain regions of rats. Cell Mol Neurobiol, 2007. 27(8): p. 985-96.

9. Bonhomme, N. and E. Esposito, Involvement of serotonin and dopamine in the mechanism of action of novel antidepressant drugs: a review. J Clin Psychopharmacol, 1998. 18(6): p. 447-54.

10. D'Souza, U.M. and I.W. Craig, Functional genetic polymorphisms in serotonin and dopamine gene systems and their significance in behavioural disorders. Prog Brain Res, 2008. 172: p. 73-98.

11. Meltzer, H.Y. and M. Huang, In vivo actions of atypical antipsychotic drug on serotonergic and dopaminergic systems. Prog Brain Res, 2008. 172: p. 177-97.

12. Adams, M.D., et al., The Genome Sequence of Drosophila melanogaster. Science, 2000. 287(5461): p. 2185-2195. 
13. Brand, A.H. and E.-L. Dormand, The GAL4 system as a tool for unravelling the mysteries of the Drosophila nervous system. Current Opinion in Neurobiology, 1995. 5(5): p. 572-578.

14. Bier, E., Drosophila, the golden bug, emerges as a tool for human genetics. Nat Rev Genet, 2005. 6(1): p. 9-23.

15. Coggan, J.S., et al., Evidence for ectopic neurotransmission at a neuronal synapse. Science, 2005. 309(5733): p. 446-51.

16. Agnati, L.F., G. Leo, A. Zanardi, S. Genedani, A. Rivera, K. Fuxe, and D. Guidolin, Volume transmission and wiring transmission from cellular to molecular networks: history and perspectives. Acta Physiologica, 2006. 187(1-2): p. 329344.

17. Park, S.K., et al., Cell-type-specific limitation on in vivo serotonin storage following ectopic expression of the Drosophila serotonin transporter, dSERT. Journal of Neurobiology, 2006. 66(5): p. 452-462.

18. Paxon, T.L., P.R. Powell, H.G. Lee, K.A. Han, and A.G. Ewing, Microcolumn separation of amine metabolites in the fruit fly. Anal Chem, 2005. 77(16): $p$. 5349-55.

19. Jackson, B.P. and R.M. Wightman, Dynamics of 5-hydroxytryptamine released from dopamine neurons in the caudate putamen of the rat. Brain Res, 1995. 674(1): p. 163-6.

20. Phillips, P.E.M., G.D. Stuber, M.L.A.V. Heien, R.M. Wightman, and R.M. Carelli, Subsecond dopamine release promotes cocaine seeking. Nature, 2003. 422(6932): p. 614-618.

21. Chen, J. and B.G. Condron, Branch architecture of the fly larval abdominal serotonergic neurons. Dev Biol, 2008. 320(1): p. 30-8.

22. Sykes, P.A. and B.G. Condron, Development and sensitivity to serotonin of Drosophila serotonergic varicosities in the central nervous system. Dev Biol, 2005. 286(1): p. 207-16.

23. Schwabe, T., R.J. Bainton, R.D. Fetter, U. Heberlein, and U. Gaul, GPCR signaling is required for blood-brain barrier formation in drosophila. Cell, 2005. 123(1): p. 133-44.

24. Bainton, R.J., L.T. Tsai, T. Schwabe, M. DeSalvo, U. Gaul, and U. Heberlein, moody encodes two GPCRs that regulate cocaine behaviors and blood-brain barrier permeability in Drosophila. Cell, 2005. 123(1): p. 145-56. 
25. MacDonald, J.M., M.G. Beach, E. Porpiglia, A.E. Sheehan, R.J. Watts, and M.R. Freeman, The Drosophila cell corpse engulfment receptor Draper mediates glial clearance of severed axons. Neuron, 2006. 50(6): p. 869-81.

26. Porzgen, P., S.K. Park, J. Hirsh, M.S. Sonders, and S.G. Amara, The Antidepressant-Sensitive Dopamine Transporter in Drosophila melanogaster: A Primordial Carrier for Catecholamines. Molecular Pharmacology, 2001. 59(1): p. 83-95.

27. Michael, D.J., J.D. Joseph, M.R. Kilpatrick, E.R. Travis, and R.M. Wightman, Improving data acquisition for fast-scan cyclic voltammetry. Anal Chem, 1999. 71(18): p. 3941-7. 


\title{
Chapter 3: Quantitative evaluation of serotonin release and clearance in Drosophila
}

\begin{abstract}
Serotonin signaling plays a key role in the regulation of development, mood and behavior. Drosophila is well suited for the study of the basic mechanisms of serotonergic signaling, but the small size of its nervous system has previously precluded the direct measurements of neurotransmitters. This study demonstrates the first real-time measurements of changes in extracellular monoamine concentrations in a single larval Drosophila ventral nerve cord. Channelrhodopsin2-mediated, neuronal type-specific stimulation is used to elicit endogenous serotonin release, which is detected using fastscan cyclic voltammetry at an implanted microelectrode. Release is decreased when serotonin synthesis or packaging are pharmacologically inhibited, confirming that the detected substance is serotonin. Similar to tetanus-evoked serotonin release in mammals, evoked serotonin concentrations are 280 - $640 \mathrm{nM}$ in the fly, depending on the stimulation length. Extracellular serotonin signaling is prolonged after administering cocaine or fluoxetine, showing that transport regulates the clearance of serotonin from the extracellular space. When ChR2 is targeted to dopaminergic neurons, dopamine release is measured demonstrating that this method is broadly applicable to other neurotransmitter systems. This study shows that the dynamics of serotonin release and reuptake in Drosophila are analogous to those in mammals, making this simple organism more useful for the study of the basic physiological mechanisms of serotonergic signaling.
\end{abstract}




\section{Introduction}

Serotonin and the serotonin transporter (SERT) are of interest due to their clinical relevance [1]. Drugs targeting SERT are used in the treatment of depression and other psychiatric illnesses. Polymorphisms affecting serotonin degradation and SERT expression have been associated with depression and anxiety [2, 3]. The basic mechanisms of serotonergic signaling are highly conserved from mammals down to $C$. Elegans [4]. Drosophila melanogaster, because of its simple nervous system, short life cycle, and ease of molecular and genetic manipulation is an attractive alternative to research on mammals [5]. While progress has been made in understanding the effects of serotonin on Drosophila behavior [6] and morphology [7], measurements of real-time serotonin release and reuptake have been hampered by a lack of analytical tools.

Quantitative evaluation of serotonin levels in the fly larvae has heretofore relied on whole-brain homogenization followed by analysis with high performance liquid chromatography [8] or capillary electrophoresis [9]. Fast scan cyclic voltammetry (FSCV) at carbon-fiber microelectrodes has been well characterized for use in mammals [10] and provides numerous advantages. The diameter of the electrodes $(7 \mu \mathrm{M})$ makes them amenable to implantation in the small larval fly nervous system. Implanted electrodes sample from the extracellular fluid, thus allowing direct measurement of the functional serotonin pool. Measurements are collected every $100 \mathrm{~ms}$, allowing rapid detection of release and clearance [11]. FSCV provides a cyclic voltammogram (CV), characteristic of the analyte detected, that aids in analyte identification [12].

Here we utilize a novel application of FSCV to quantify changes in extracellular monoamines in the isolated fly larval ventral nerve cord (VNC). Channelrhodopsin2(ChR2) mediated stimulation produces neuron-specific induction and physiologicallyrelevant release. Pharmacological agents that inhibit serotonin synthesis and packaging confirm the measured compound is serotonin and that release is vesicular. We show 
that the dynamics of serotonin release and reuptake in Drosophila are analogous to those in mammals and that transport is involved in the clearance of serotonin from the extracellular space. The ability to monitor release and transporter function in real-time significantly strengthens the utility of Drosophila as a model system for studying the basic mechanisms underlying neurotransmission.

\section{Materials and Methods}

\section{i. Instrumentation and electrochemistry}

Carbon-fiber microelectrodes were manufactured from single T-650 carbon fibers, $7 \mu \mathrm{m}$ in diameter (Cytec Engineering Materials, West Patterson, NJ), as previously described [13]. A Dagan ChemClamp potentiostat was used to collect electrochemistry data (Dagan, Minneapolis, MN; custom-modified). Data acquisition software and hardware were the same as described by Heien et al [14]. For dopamine detection, the electrode potential was continuously scanned from -0.4 to $1.3 \mathrm{~V}$ and back at $600 \mathrm{~V} / \mathrm{s}$ every $100 \mathrm{~ms}$, even when data was not being collected. For serotonin detection, we applied a modified waveform, from $0.2 \mathrm{~V}$ to $1.0 \mathrm{~V}$, then to $-0.1 \mathrm{~V}$ then back to $0.2 \mathrm{~V}$ at a slew rate of $1,000 \mathrm{~V} / \mathrm{s}$ every $100 \mathrm{~ms}$ [15]. A silver-silver chloride wire was used as a reference electrode. Electrodes were calibrated with $1 \mu \mathrm{M}$ serotonin or $1 \mu \mathrm{M}$ dopamine prior to and after use in situ. A small percentage of electrodes were discarded because they exhibited a signal to noise ratio of less than 100 or were insufficiently sensitive, with less than $6 \mathrm{nA}$ of oxidative current for $1 \mu \mathrm{M}$ serotonin or dopamine.

\section{ii. Fly stocks}

Flies containing Channelrhodopsin-2 (a gift from Christian Schroll, Universitat Wurzburg) were crossed to flies expressing TH-GAL4 or Tph-GAL4 (a gift from Jaeson Kim, Korea Advanced Institute of Science and Technology) to generate homozygous 
lines with the following genotypes: Tph-GAL4; UAS-ChR2 and UAS-ChR2; TH-GAL4. Canton S (CS) flies, used as a control, were obtained from Bloomington stock center (http://flystocks.bio.indiana.edu/). Larvae were allowed to feed on yeast supplemented with $10 \mathrm{mM}$ all-trans retinal (Sigma-Aldrich, St. Louis) for 2-3 days prior to the initiation of experiments while protected from light.

\section{iii. Preparation of ventral nerve cords (VNCs)}

5 day old larval brains were dissected in modified Schneider's insect media (15.2 $\mathrm{mM} \mathrm{MgSO} 4,21 \mathrm{mM} \mathrm{KCl}, 3.3 \mathrm{mM} \mathrm{KH}_{2} \mathrm{PO} 4,53 \mathrm{mM} \mathrm{NaCl}, 5.8 \mathrm{mM} \mathrm{NaH}_{2} \mathrm{PO}_{4}, 5.4 \mathrm{mM}$ $\mathrm{CaCl}_{2}, 11.1 \mathrm{mM}$ Glucose, $5.3 \mathrm{mM}$ Trehalose, $\left.\mathrm{pH}=6.2\right)$. The composition of this media allows the long-term culture of isolated VNCs and the electrochemical detection of serotonin. The optic lobes were removed by means of a horizontal cut in the anteriormost portion of the VNC, which was then placed, neuropil side down, onto the bottom of a petri plate in $3 \mathrm{ml}$ fresh buffer. An electrode was implanted using a micromanipulator, while the VNC was visualized under a 40X water immersion objective. The manipulator was angled so as to place the electrode within the middle portion of the neuropil when the electrode tip was inserted to a distance of 4-6 segments away from the cut edge (A diagram of this is shown in chapter 2, figure 1). Experiments were not initiated until at least 5 min after electrode implantation to allow the electrode background current to stabilize.

\section{iv. Data collection}

Data collection was initiated no later than 50 minutes following VNC isolation. No significant changes in signal peak height or time to half maximal signal decay resulted from changes in wait time within this window (Figure 1). After the collection of at least 30 seconds of baseline data, VNCs were exposed to 10 seconds of intense blue light to 
stimulate release. The light source was a 10W halogen microscope bulb with a standard fluorescein emission filter (450 - $490 \mathrm{~nm})$ that was manually-switched. VNCs were subsequently allowed to rest in the dark for 5-10 minutes before the next stimulation. Peak height remained stable when 10 s long stimuli were performed 5 min apart (data not shown) and this inter-stimulation time was used for variable length stimulation experiments.

a. Size of initial peak vs. time from dissection to start of experiment in Tph; ChR2 VNCs

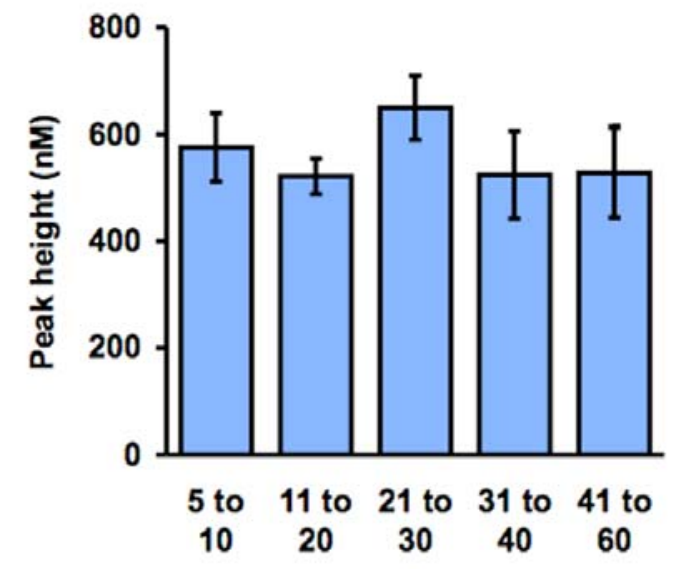

Time from dissection to start of experiment $(\mathrm{min})$ a. Duration of initial peak vs. time from dissection to start of experiment in Tph; ChR2 VNCs

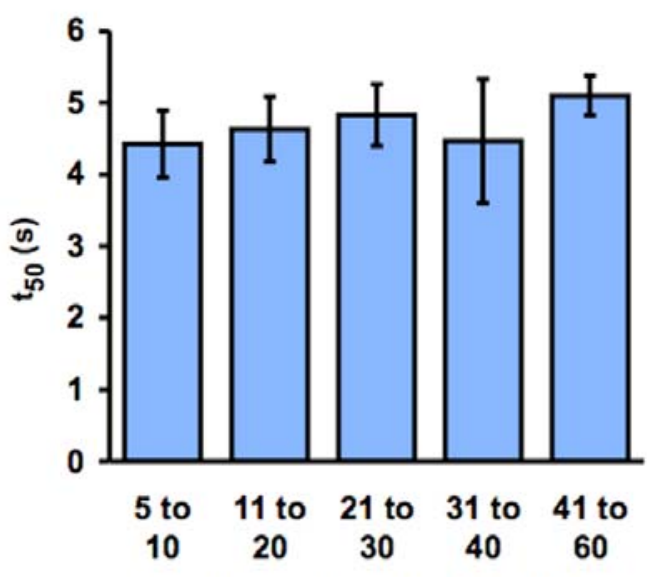

Time from dissection to start of experiment (min)

Figure 1. Serotonin signal remains stable over a 60 minute window following isolation of the ventral nerve cord (VNC). During experiments involving pharmacologic manipulation, dissected VNCs were allowed to incubate in buffer containing the drug for 20-30 minutes to allow for drug diffusion. As a control, drug-naïve VNCs were incubated in buffer for variable lengths of time prior to blue light stimulation. (a,b) Pooled data (mean +/- SEM, $\mathrm{n}=5-12$ ) showing that peak height (a) and time to half maximal signal decay (t50) (b) of signals remain stable over 60 minutes following dissection. There are no significant differences between categories $(0.33<\mathrm{p}<0.97$, student t-tests, two tailed).

The following drugs were purchased from Sigma-Aldrich: Reserpine, Fluoxetine hydrochloride, 4-Chloro-DL-phenylalanine (PCPA), and cocaine hydrochloride. In 
experiments involving pharmacological manipulations, VNCs were dissected and incubated in the presence of drug for 20-30 minutes before stimulation to allow time for drug diffusion.

Drug effects on electrode sensitivity to dopamine had been previously reported [16]. Therefore, a series of in vitro experiments were conducted in a flow cell to assay the impact of local drug concentrations on serotonin detection by FSCV. The results of these experiments were part of the initial characterization of serotonin detection by FSCV and are therefore presented in the methods section on this document. Buffer was pumped through the flow injection apparatus as previously described in Swamy and Venton, 2007 [17]. The electrode, positioned at the outlet of this apparatus, was exposed to $500 \mathrm{nM}$ serotonin for 10 s to mimic the length and size of exposure expected in situ. Peak current readings were averaged from 3 consecutive exposures prior to, 10 minutes after the electrode was bathed in drug, and finally 10 minutes after drug washout. Readings were normalized to the size of the initial, pre-drug signal for each electrode.

The results shown in Figure 2 demonstrate that exposure to $10 \mu \mathrm{M}$ fluoxetine, 10 $\mu \mathrm{M}$ cocaine, and $100 \mu \mathrm{M}$ reserpine decrease electrode sensitivity to serotonin while 100 $\mu$ M PCPA does not. The effects partially reverse after a 10 minute drug washout. Although sensitivity was reduced by as much as $50 \%$ in the case of reserpine, $500 \mathrm{nM}$ serotonin still produced a large deviation in signal with greater than 1:100 signal to noise ratio and therefore this effect would be unlikely to prevent the detection of serotonin signals in situ. Furthermore, drugs effects on sensitivity were accounted for in all experiments in this paper during electrode calibration because all solutions contained the drug. It was assumed that after 20 minutes of incubation time, the drug concentration within the VNC was similar to that of the bath solution. Drug effects on electrodes would pose a larger problem for feeding experiments where the tissue drug concentration is unknown. 
a. Before drug exposure

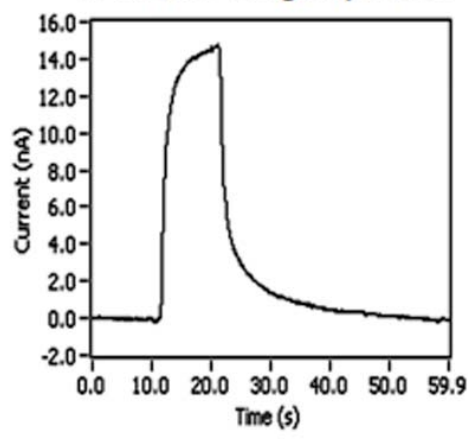

b. In presence of $10 \mu \mathrm{M}$ coc.
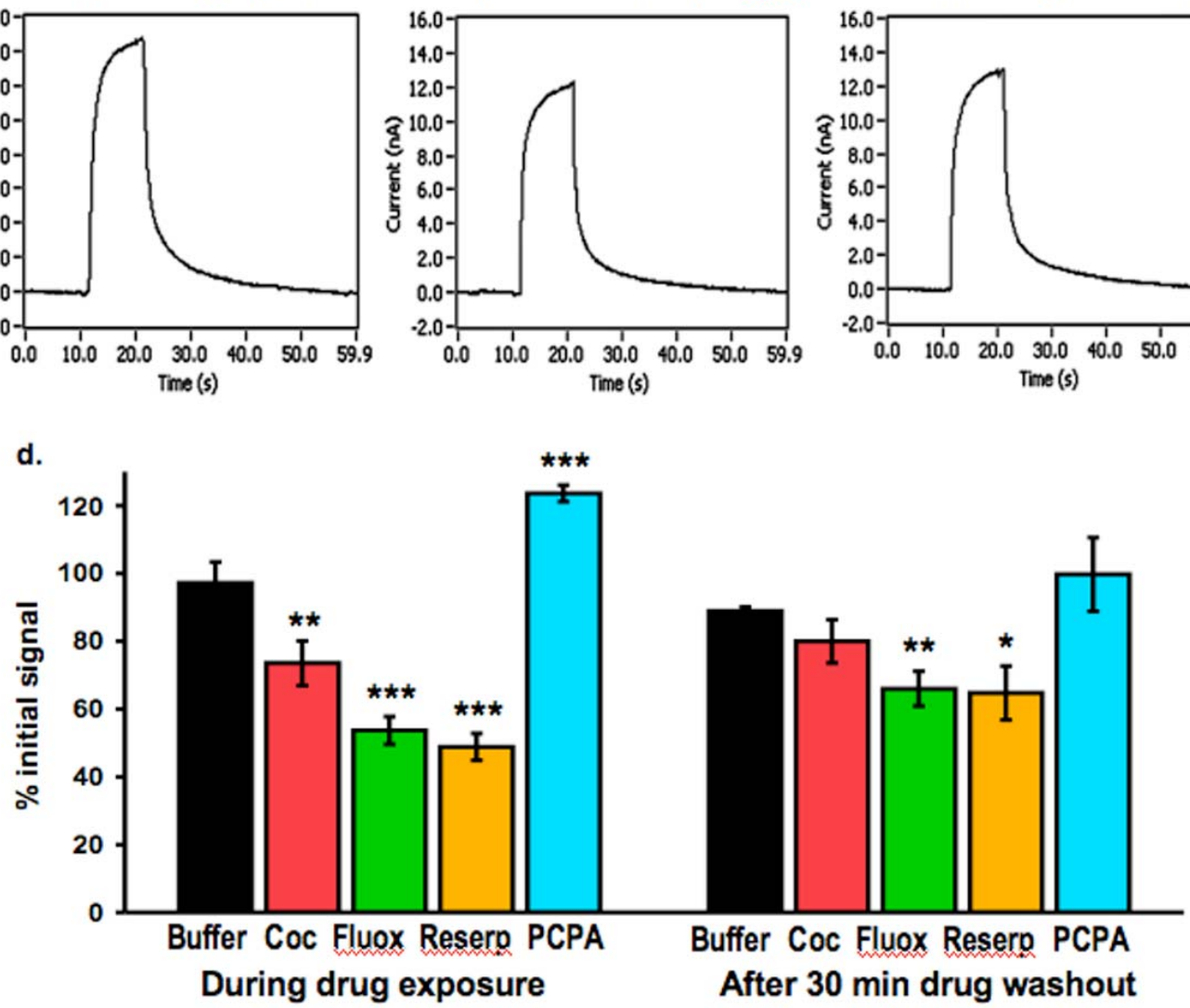

Figure 2. To determine the effect of local drug concentrations have on electrode sensitivity to serotonin, electrodes were exposed to serotonin in a flow cell. (a-c)

Representative current traces from an electrode exposed to $500 \mathrm{nM}$ serotonin for $10 \mathrm{~s}$ in buffer (a), in buffer containing $10 \mu \mathrm{M}$ cocaine (b), and in buffer $10 \mathrm{~m}$ after cocaine has washed out (c). (d) pooled results, mean +/- SEM, for 5 electrodes. Readings normalized to the size of the initial pre-drug signal. Asterixes denote significant difference from signal in buffer $\left({ }^{*} p<0.05,{ }^{* *} p<0.01,{ }^{* * *} p<0.001\right)$. Exposure to cocaine, fluoxetine, or reserpine leads to a $35,45 \%$, or $50 \%$ decrease in signal while PCPA exposure does not decrease the signal.

\section{v. Data analysis}

Electrochemical data was analyzed using Tar Heel CV software [18]. Data were visualized as a background-subtracted cyclic voltammogram (CV), a concentration vs time trace, or a 3D color plot showing all the data. For a detailed description of 
monoamine detection by FSCV, see Figure 3. All CVs and color plots shown in this paper have been background subtracted by averaging 10 scans collected 1 second before blue light exposure. Signal CVs were collected approximately 1 second after the cessation of blue light exposure. CVs were used to identify the voltage corresponding to the maximum serotonin or dopamine oxidation peaks. The current at this voltage was converted to serotonin concentration using post calibration data for that electrode, and these changes in concentration over time are plotted as the signal trace.

To aid in electrochemical identification, the CV obtained during electrode calibration was compared to the $\mathrm{CV}$ from the $\mathrm{VNC}$. The mean $\mathrm{R}^{2}$ for a linear regression fit between calibration and signal CVs for 10 electrodes was $0.82+/-0.02$. The reasons for differences between calibration and VNC data include different ionic concentrations inside the tissue and a calibration method that results in small changes in the background capacitance due to changing fluid levels. Additionally, minor shifting apart of the oxidation and reduction peaks occurs as a result of slightly slower kinetics in situ. Less than $5 \%$ of samples were excluded because of poor electrode placement or electrode drift. VNCs were excluded if the shape of the background current in the VNC did not match that of the background current in the buffer. This happened when the electrode was not implanted in the neuropil or remained attached to the glial outer layer and resulted in a background that was more triangular shaped than normal. VNCs were also excluded if the electrode had severe drift because drift can cause errors in measuring peak duration. Severe background drift was defined as a change in baseline current of more than $1.5 \mathrm{nA}$ in 100s. Greater amounts of drift and larger peak shifts were observed when using the dopamine waveform. Statistical analysis of pooled data including two-tailed student t tests was conducted using Excel and InStat software. Curve fitting was done in GraphPad Prism. 

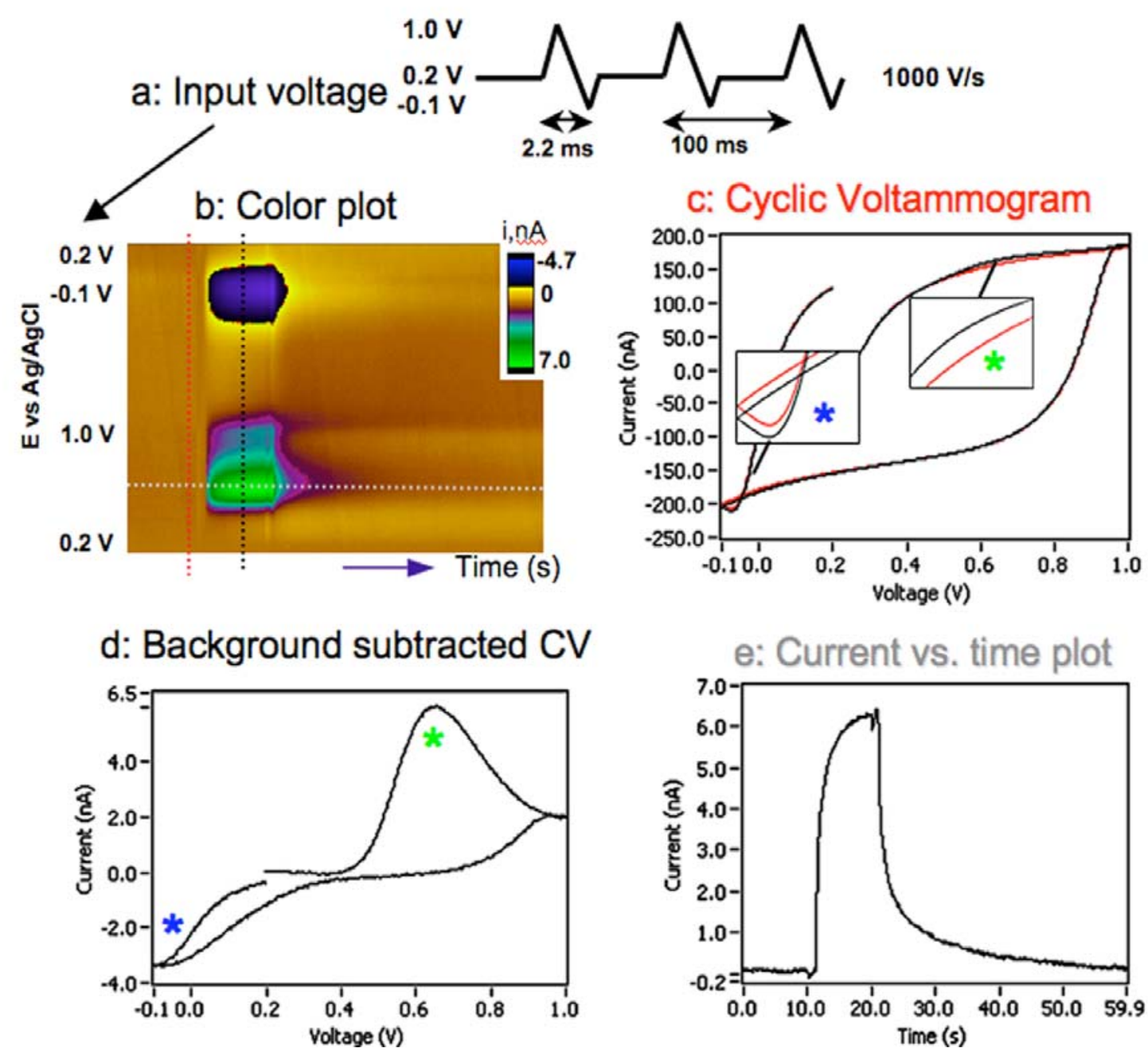

Figure 3. Detecting serotonin with fast scan cyclic voltammetry. Data from flow cell experiment showing electrode response to a 10 second exposure of $1 \mathrm{mM}$ serotonin in buffer. (a) A waveform from 0.2 to $1.0 \mathrm{~V}$ to $-0.1 \mathrm{~V}$ to $0.2 \mathrm{~V}$ is applied at $1000 \mathrm{~V} / \mathrm{s}$ every $100 \mathrm{msec}$. (b) The resulting signal is visualized as a 3D color plot, combining applied voltage $(y)$, time $(x)$, and current output (color). Cutting along the $x$ axis provides a plot of current vs. voltage, the cyclic voltammogram (CV). (c) CVs of signal prior to (red) and during (black) serotonin exposure taken at times denoted by red and black dashed lines on $\mathrm{b}$. The shape of the $\mathrm{CV}$ is a combination of background charging and smaller faradaic currents of the species of interest. Because of the temporal stability of the background charging current, small changes in signal resulting from serotonin exposure (magnified in the insets) can be measured by means of background subtraction. All subsequent CVs shown in this study are background subtracted. (d) A background subtracted CV shows the main oxidation peak for serotonin $\left(^{*}\right)$ at $0.6 \mathrm{~V}$. The main reduction $\left(^{*}\right)$ peak is evident 
at $\mathrm{OV}$. The shape of the CV, particularly the location of the oxidation and reduction peaks, is used to identify the electroactive species. The location of the oxidation peak on the $\mathrm{CV}$ is used to determine where to cut along the $\mathrm{y}$ axis of the color plot to provide a plot of current vs. time (e). The response of the electrode to an injection of serotonin is shown in (e). Current is subsequently converted to concentration using electrodespecific calibration data for the fly experiments.

\section{Results}

i. Characterization of ChR2-mediated serotonin and dopamine release in the fly

To provide neuron-specific stimulation, we expressed ChR2, a blue lightactivated, cation-selective ion channel from the green algae Chamydomonas reinhardtii [19]. ChR2 allows neural excitation on a millisecond timescale and provides single action potential control of neuronal signaling. Flies containing ChR2 under the control of a GAL4 binding upstream activator sequence (UAS) were crossed to Tph-GAL4 or THGAL4 "driver" lines to provide serotonergic or dopaminergic-specific expression, respectively. Tryptophan hydroxylase (Tph) and Tyrosine hydroxylase (TH) are the ratelimiting enzymes in the biosynthesis of serotonin and dopamine [20]. Electrodes were implanted into the neuropil of isolated, intact Drosophila VNCs from wandering third instar larvae, which exhibit a mature, fully-developed serotonergic system [7].

To detect serotonin, we applied a modified waveform, from a holding potential of $0.2 \mathrm{~V}$ to $1.0 \mathrm{~V}$, then to $-0.1 \mathrm{~V}$ and back to $0.2 \mathrm{~V}$ at $1000 \mathrm{~V} / \mathrm{s}$ every $100 \mathrm{~ms}$. This waveform was previously optimized for the sensitive detection of serotonin, provides a 10-fold greater electrode sensitivity for serotonin over dopamine, and alleviates electrode fouling by oxidized serotonin [15]. Untreated electrodes were used instead of Nafion coated electrodes to facilitate fast electrode response times. Figure 4 a-c shows that a ten second duration blue-light stimulation of a Tph-GAL4; UAS-ChR2 VNC elicits serotonin release. The green and blue areas on the color plot correspond to the 
oxidation and reduction of serotonin (Fig. 4a). The background-subtracted CV (Fig. 4b) confirms that serotonin was detected because the peak locations for the larval CV (black line) are similar to the $1 \mu \mathrm{M}$ serotonin calibration $\mathrm{CV}$ (blue line). The concentration vs time trace shows that detected changes in serotonin are time-locked to the stimulus (Fig $.4 c)$.

Serotonin release was not detectable in any of the control VNCs including those from the parental strains UAS-ChR2 and Tph-GAL4, as well as Canton S (CS). For example, in Figure 4d the color plot from a CS VNC shows minor fluctuations across voltages during the stimulation, with the largest change occurring at the switching potential $(1.0 \mathrm{~V})$. No characteristic oxidation or reduction peaks for serotonin are apparent in the CV (Fig. 4e). The concentration vs. time trace, at the potential for serotonin oxidation, in Figure $4 \mathrm{f}$ shows that the minor noise fluctuation corresponds to about $70 \mathrm{nM}$ serotonin. Pooled data $(n=4)$ shows that on average, this error is about $11 \%$ of the normal peak serotonin detected.

To detect dopamine, we applied a standard, triangle waveform from -0.4 to $1.3 \mathrm{~V}$ and back at a slew rate of $600 \mathrm{~V} / \mathrm{s}$ every $100 \mathrm{~ms}$. This waveform exhibits enhanced electrode sensitivity to dopamine [14]. Stimulation of a TH-GAL4; UAS-ChR2 VNC evoked dopamine release, as evident in the color plot (Fig. 4g) and signal CV (Fig. 4h). Dopamine release was also time-locked to the stimulation (Fig. 4i). No dopamine was observed in a control VNC from a larva expressing only TH-gal4 but no UAS-ChR2 (Fig. 4j). As seen with the serotonin control experiments, minor changes are observed across voltages but no peaks corresponding to the oxidation or reduction peaks of dopamine are visible (Fig. 4k). This results in an error of about $60 \mathrm{nM}$ (Fig. 4l) 

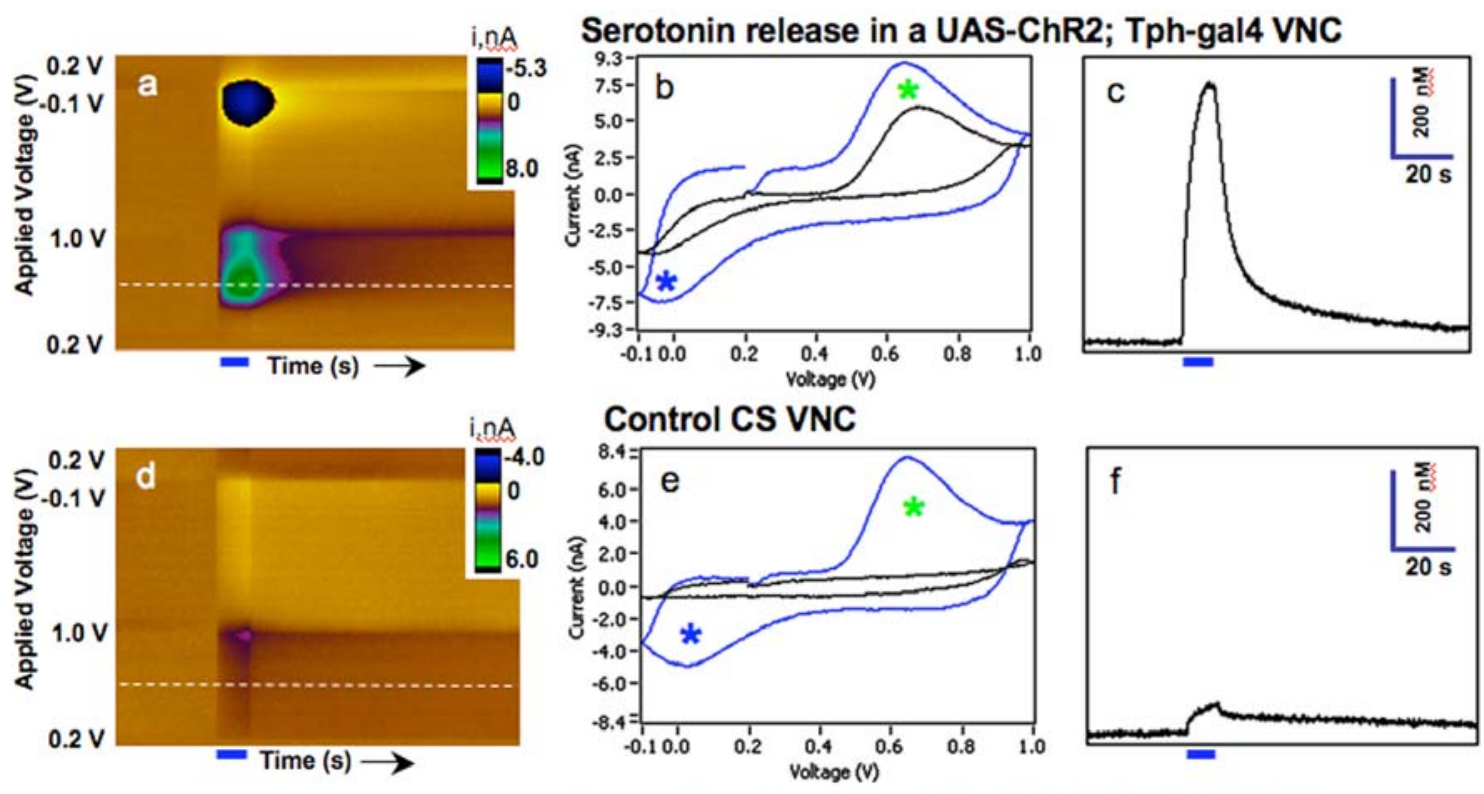

\section{Control CS VNC}
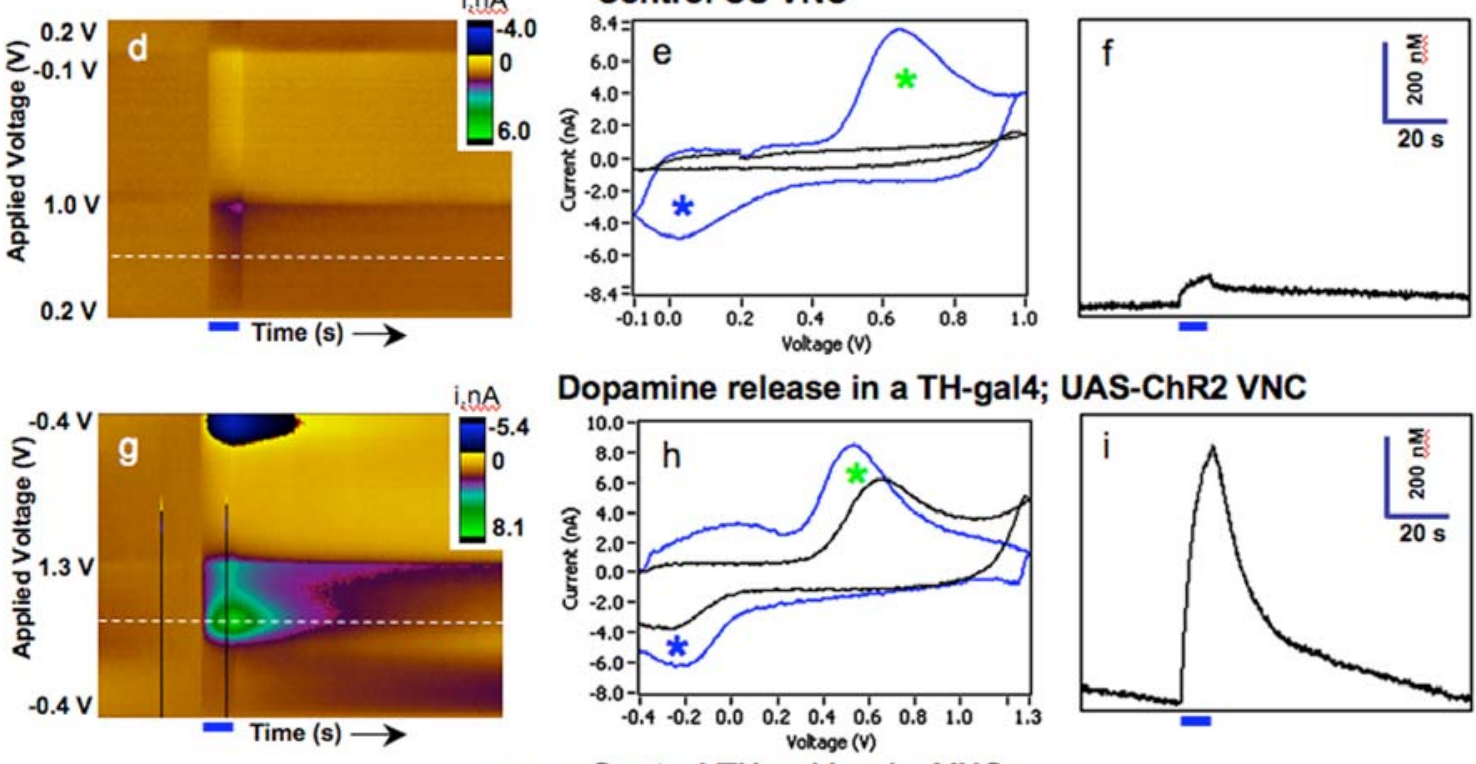

Dopamine release in a TH-gal4; UAS-ChR2 VNC
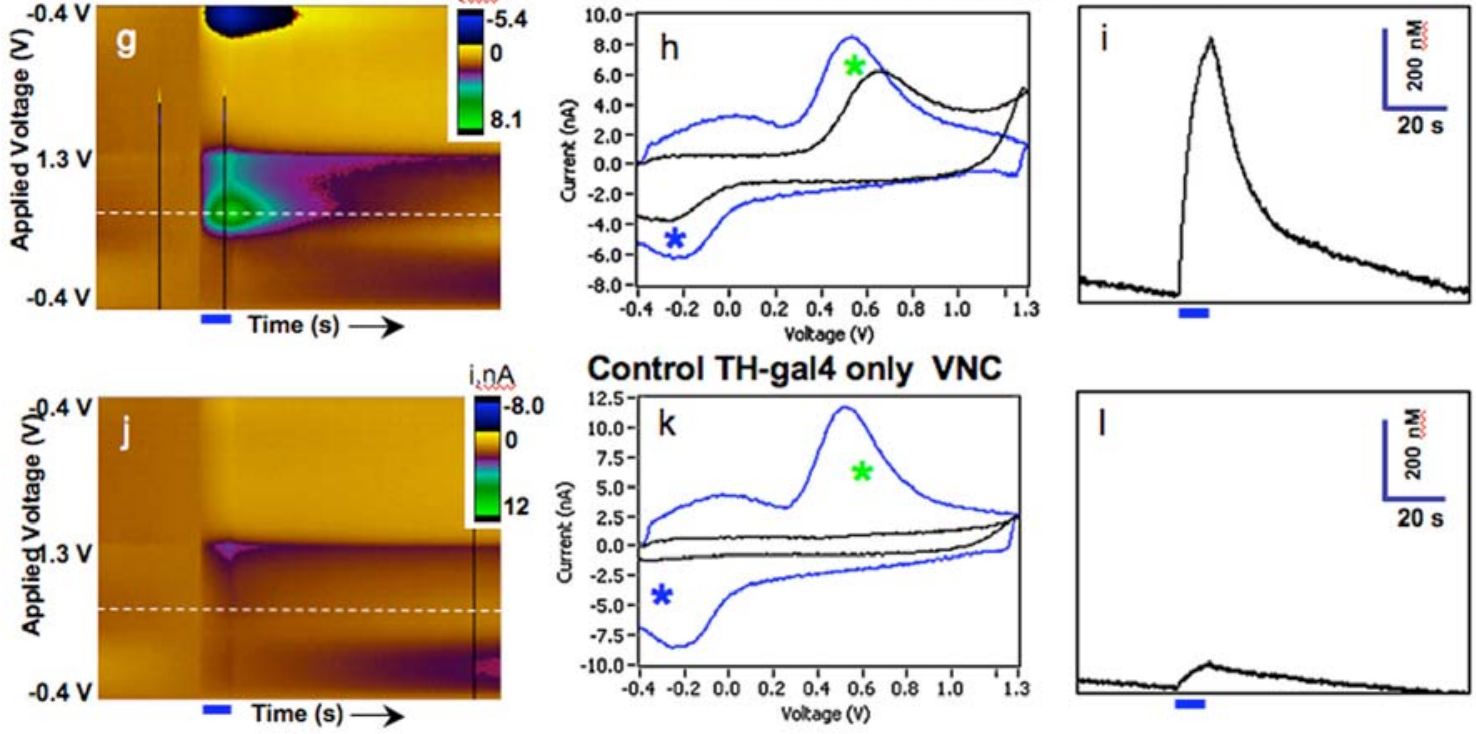

\section{Control TH-gal4 only VNC}

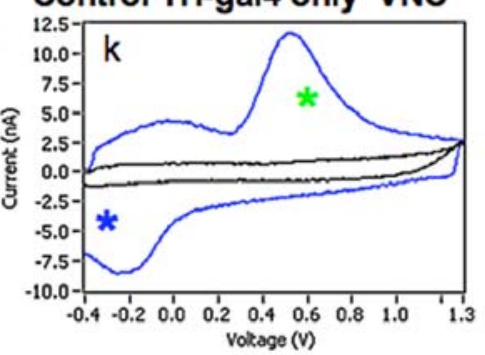

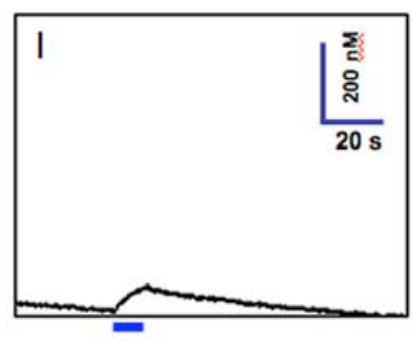

Figure 4. Characterization of evoked serotonin and dopamine signals. Blue light stimulation of isolated larval Drosophila VNCs elicits depolarization in neurons expressing ChR2. Left column: Color plots with dashed white line denoting oxidation potential utilized for signal trace. Duration of blue light exposure (10 s) indicated by blue bars. Color plots are scaled so the maximum oxidative current corresponds to $750 \mathrm{nM}$ monoamine. Middle: The CVs verify the compound being detected. The CV from the fly (black line) is compared to that obtained during electrode calibration with $1 \mu \mathrm{M}$ monoamine (blue line). The asterisks identify the oxidation and reduction peaks, which correspond to the green and blue areas in the color plots, respectively. Right: Signal 
traces show neurotransmitter concentration changes over time. Currents were converted to concentration based on electrode calibration values. (a-c) Serotonin release from a VNC expressing ChR2 under the control of Tph-GAL4. The color plot (a) and CV (b) exhibit serotonin-specific peaks as evident by the signal CVs match to the calibration CV. The signal trace (c) shows the serotonin peak is time-locked to the stimulus. Data from a control VNC lacking ChR2 expression shows minor fluctuations throughout the color plot (d) upon blue light exposure but the CV (e, black line) does not show any neurotransmitter specific peaks. (f) Trace shows a small error fluctuation in current.(g-i) Dopamine release from a VNC expressing ChR2 under the control of TH-GAL4. Note a different voltage waveform was used than for serotonin. The color plot (g) and CV (h) verify that dopamine was detected. Dopamine release is also time-locked to the stimulation (i). Data from a control VNC lacking ChR2 expression shows changes similar to those observed with the other waveform. Minor fluctuations are present at all voltages during the stimulation (j) but the CV (k) does not show any neurotransmitter specific peaks. (I) Trace shows a small fluctuation in current.

The GAL4-UAS system allows specific stimulation of one type of neuron, so release should be predominantly either dopamine or serotonin. While distinguishing serotonin from dopamine solely based on electrochemistry is difficult, previous studies have used cyclic voltammetry for codetection of serotonin and dopamine [21]. The dopamine CV has a greater separation between oxidation and reduction peaks and the positions of the dopamine and serotonin reduction peaks are far enough apart to allow them to be separated. Consistent with this, no serotonin-specific reduction peaks were observed after stimulation of VNCs expressing ChR2 in dopaminergic neurons. Because the electrode is more sensitive to serotonin, the reduction peak would be expected to be detected if serotonin had been present.

The peak serotonin concentration detected varies with the duration of blue light exposure (Fig. 5). Traces from a representative VNC show the relative size of the peaks elicited by 2, 5, 10, or $30 \mathrm{~s}$ stimulation (Fig. 5a). These stimulations elicited $280-640 \mathrm{nM}$ 
serotonin. The two second-long stimulation elicits roughly half the peak serotonin concentration as seen with $10 \mathrm{~s}$. The peak concentration for a $10 \mathrm{~s}$ stimulation is about equal to that using $30 \mathrm{~s}$, although the duration of the signaling is longer with the longer stimulation. Therefore, uptake probably acts to clear some of the serotonin during the stimulation, leading to a steady-state concentration. Because the light source was manually operated, stimulation lengths less than 2 s were difficult to achieve. Pooled data shows that maximal serotonin concentration appears to reach a plateau at $10 \mathrm{~s}$ (Fig. 5b) and the peak height for $30 \mathrm{~s}$ stimulations was not significantly different than $10 \mathrm{~s}$ stimulations. We chose to use $10 \mathrm{~s}$ long stimulations for the rest of the experiments that characterize serotonin release and clearance.
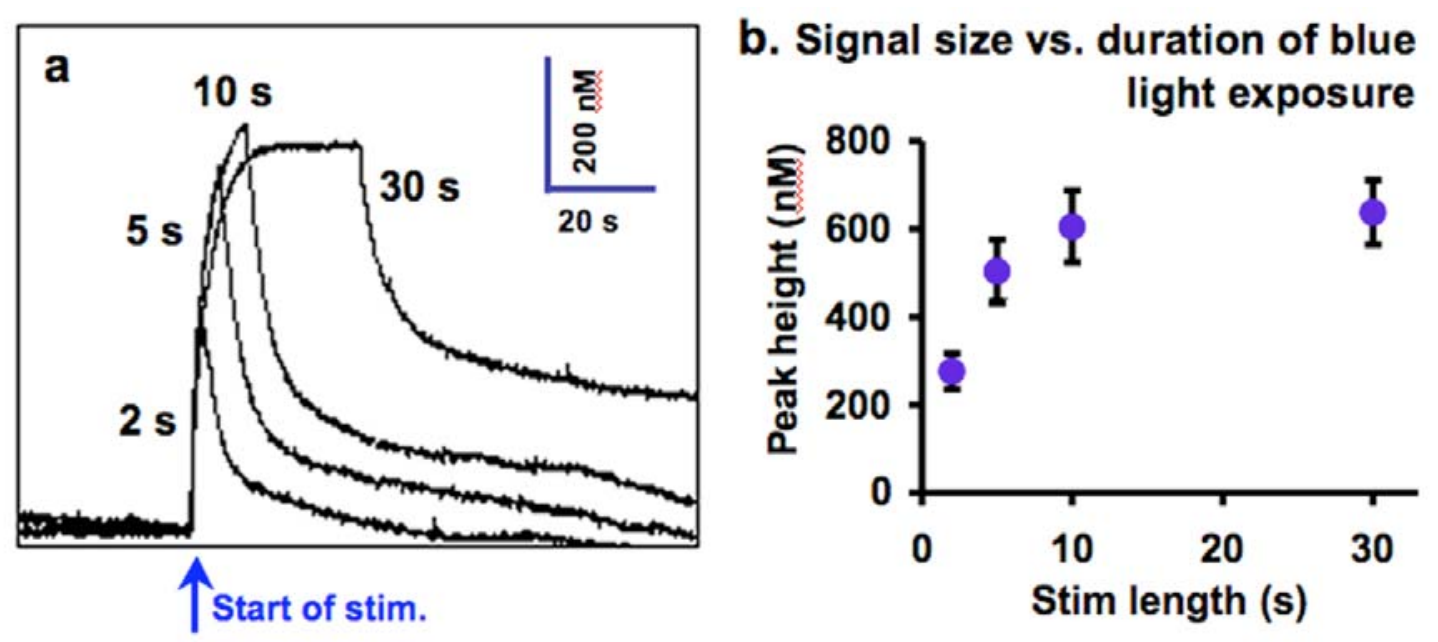

Figure 5. Evoked peak serotonin concentration varies with blue light stimulus duration. (a) Representative traces showing the effect of different stimulation lengths $(2,5,10$, and 30s) on peak height in the same sample. (b) Pooled data (mean +/- SEM, $n=6$ samples) shows an increase in peak height with increasing duration of blue light exposure. Peak height appears to reach a plateau after 10s; peak height at $30 \mathrm{~s}$ is not significantly different from that at $10 \mathrm{~s}$ ( $p=0.78$, student t-test, 2 tailed). 
ii. Release is due to vesicular serotonin

To quantify evoked signals, peak height (maximal concentration) and the time for half maximal signal decay $\left(t_{50}\right)$ were calculated. A diagram of the measured parameters is shown in Figure 6a. When VNCs are dissected in buffer, signals remain relatively stable for 90 min when 10s long stimulations are performed 10 min apart (Fig. 6b). Pooled data shows the relative stability of both the peak height and $t_{50}$ over the course of 8 stimulations (Fig. 6 c,d).

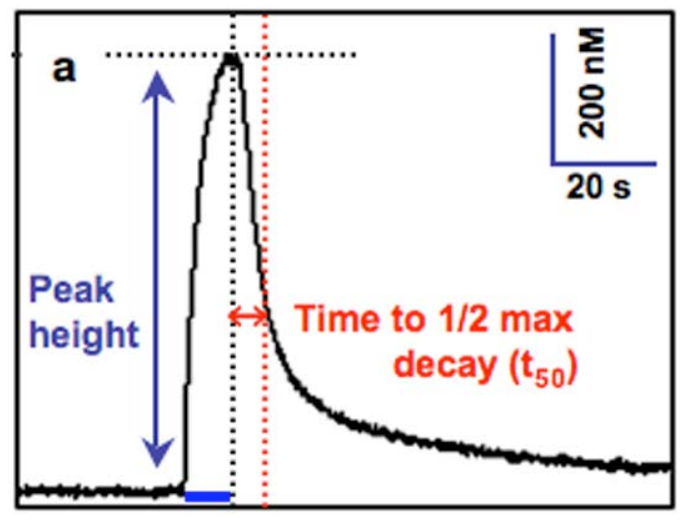

c. Serotonin clearance

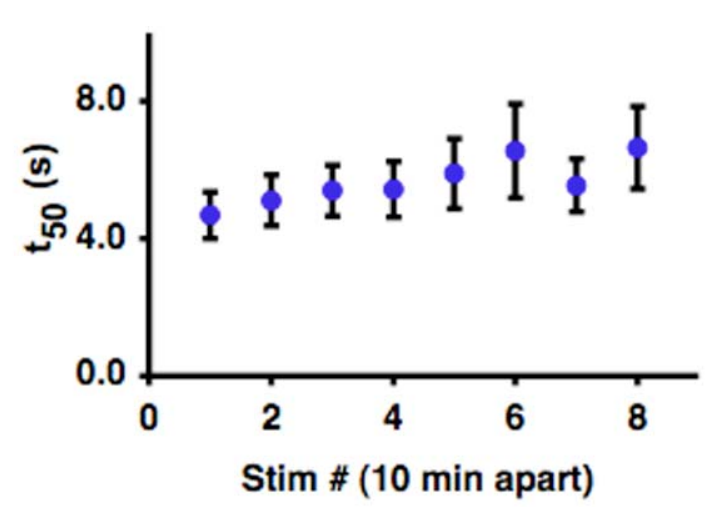

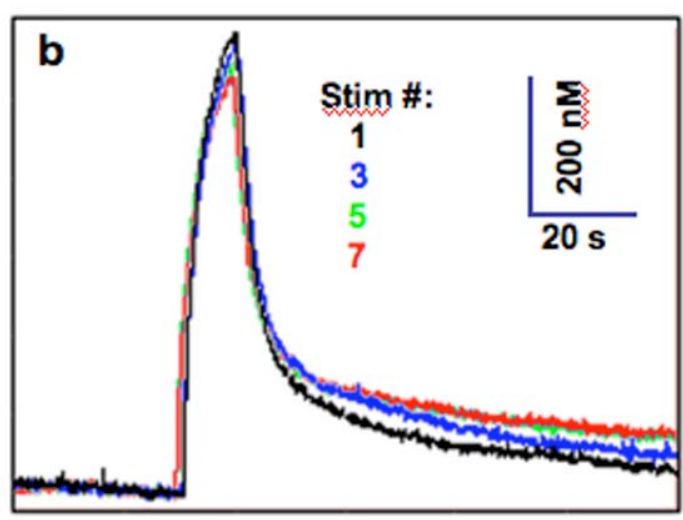

d. Peak height

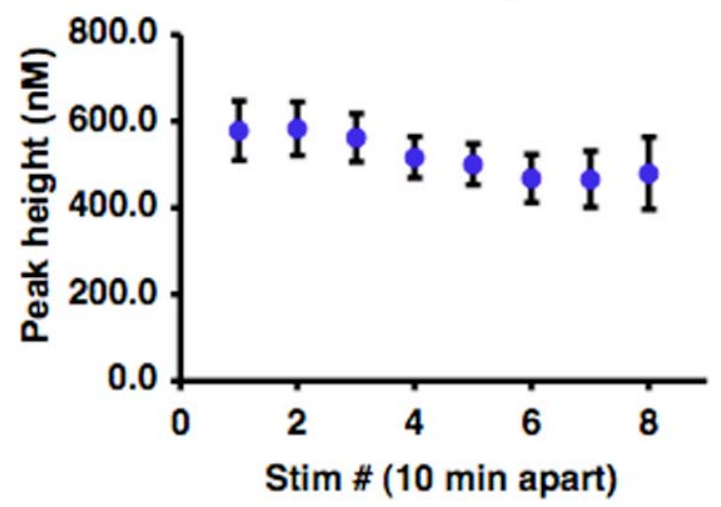

Figure 6. Stability of serotonin signal. (a) Diagram of measured parameters. Peak height, which is the maximal concentration change from baseline, and time to half maximal signal decay ( $\mathrm{t50}$ ), which is the time from the end of the blue light stimulation until the signal decays to half its maximal value, were calculated. (b) Representative traces from same VNC showing repeated stimulations. All data are 10s long stimulations performed 10 min apart. (c,d) Pooled data (mean +/- SEM, $n=4$ ) from VNCs dissected 
in buffer showing that $t 50$ (c) and peak height (d) remain relatively stable over 8 stimulations repeated 10 min apart.

Although electrochemical evidence strongly suggests that the observed signal is serotonin, we chose not to rely on electrochemical identification as the sole evidence of serotonin detection. We therefore conducted experiments to verify the nature of the analyte by pharmacologically disrupting the synthesis or packaging of serotonin into vesicles. When VNCs are incubated for $30 \mathrm{~min}$ in the serotonin synthesis inhibitor PCPA, the peak height decreases for later stimulations. Representative traces from a VNC dissected in $100 \mu \mathrm{M}$ PCPA are superimposed in Figure 7a to highlight the decreasing peak height. Pooled data shows that while the initial stimulation is similar to controls, the peak height with PCPA decreases to $50+/-3 \%$ of the initial peak height by the forth stimulation (Fig. 7b). The decrease in signal with synthesis inhibition confirms the identity of the electroactive species as serotonin.

To test if release was from vesicular serotonin, the packaging of serotonin into vesicles by the vesicular monoamine transporter (VMAT) was inhibited with reserpine. Blue light stimulation of VNCs incubated in $100 \mu \mathrm{M}$ reserpine for 30 minutes did not elicit detectable serotonin release. Color plots and CVs from reserpine-incubated VNCs did not show any peaks consistent with serotonin detection. A representative trace from a VNC incubated in reserpine is superimposed on one from a control VNC lacking ChR2 expression to highlight that the small change is due to noise seen in all VNCs and not serotonin (Fig. 8a). Pooled data (Fig. 9c) show that peak height with reserpine is not significantly different from control VNCs lacking ChR2 expression. These data show that serotonin release is vesicular. 


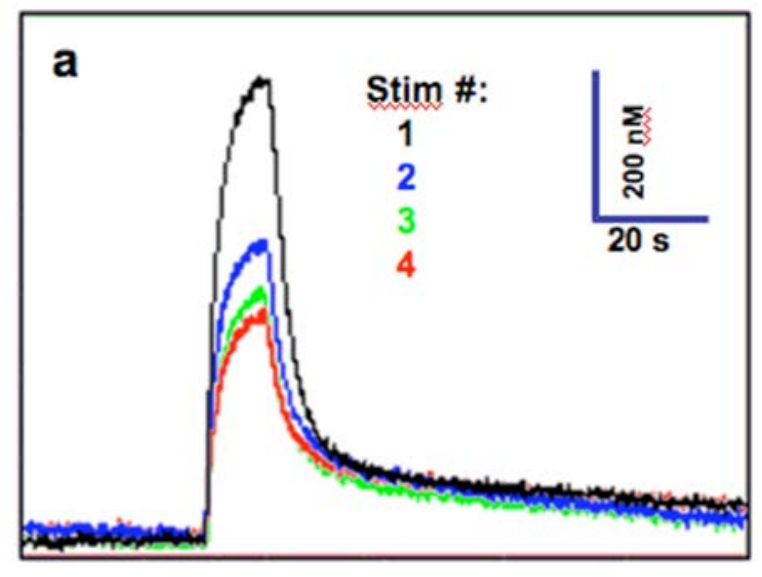

b. Effect of PCPA on peak height

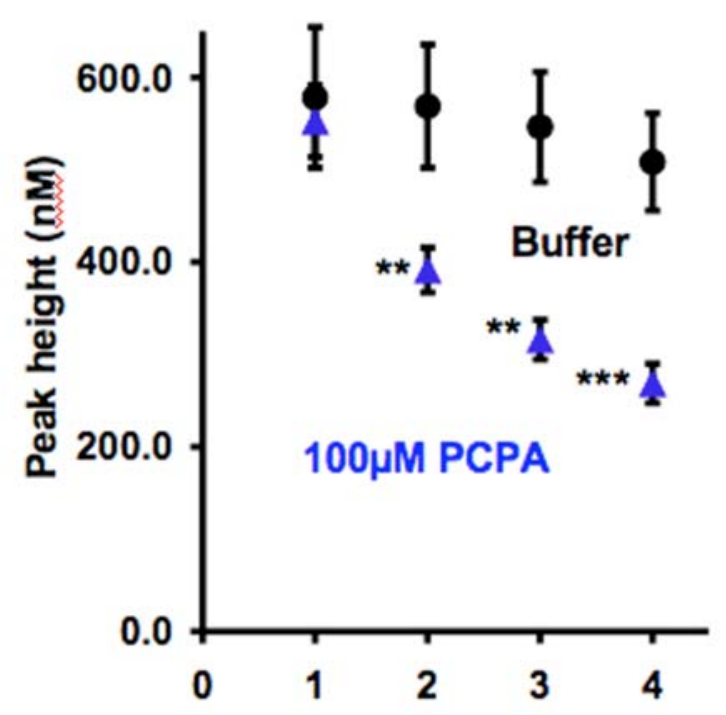

Figure 7. Effect of PCPA on serotonin release. When VNCs are dissected in the serotonin synthesis inhibitor PCPA, serotonin release decreases with repeated stimulation. All data is from 10 $s$ long stimulations performed $10 \mathrm{~min}$ apart. (a) Representative traces from a VNC that has been incubated in PCPA, superimposed to show progressive decrease in peak height. (b) Pooled data (mean +/-SEM, $\mathrm{n}=6$ ) showing that peak height in PCPA incubated VNCs (triangles) decreases to $50+/$ $3 \%$ of initial value by the $4^{\text {th }}$ stimulation. Asterisks indicate data are significantly different $\left({ }^{* *} \mathrm{p}<0.01,{ }^{* \star *} \mathrm{p}<0.001\right.$, student t-test, 2 tailed) than samples incubated in buffer (circles).

\section{Stim \#}

iii. Characterization of serotonin clearance in the fly

We expect that released serotonin is eliminated primarily through reuptake by SERT, since Drosophila do not contain significant levels of monoamine oxidase, the enzyme responsible for breakdown in higher animals [9]. To test this hypothesis, we blocked transporter function with $10 \mu \mathrm{M}$ cocaine or $10 \mu \mathrm{M}$ fluoxetine. After reuptake inhibition, serotonin clearance is slower and signal takes longer to return to baseline (Fig. 9b). No additional peaks are detected after either cocaine or fluoxetine (Fig. 8). Pooled data show that transporter inhibition does not affect the peak evoked serotonin 
concentration (Fig. 9c) but does significantly increase $t_{50}$ by about 3 times (Fig. 9d). This parameter therefore correlates to the rate of serotonin reuptake.
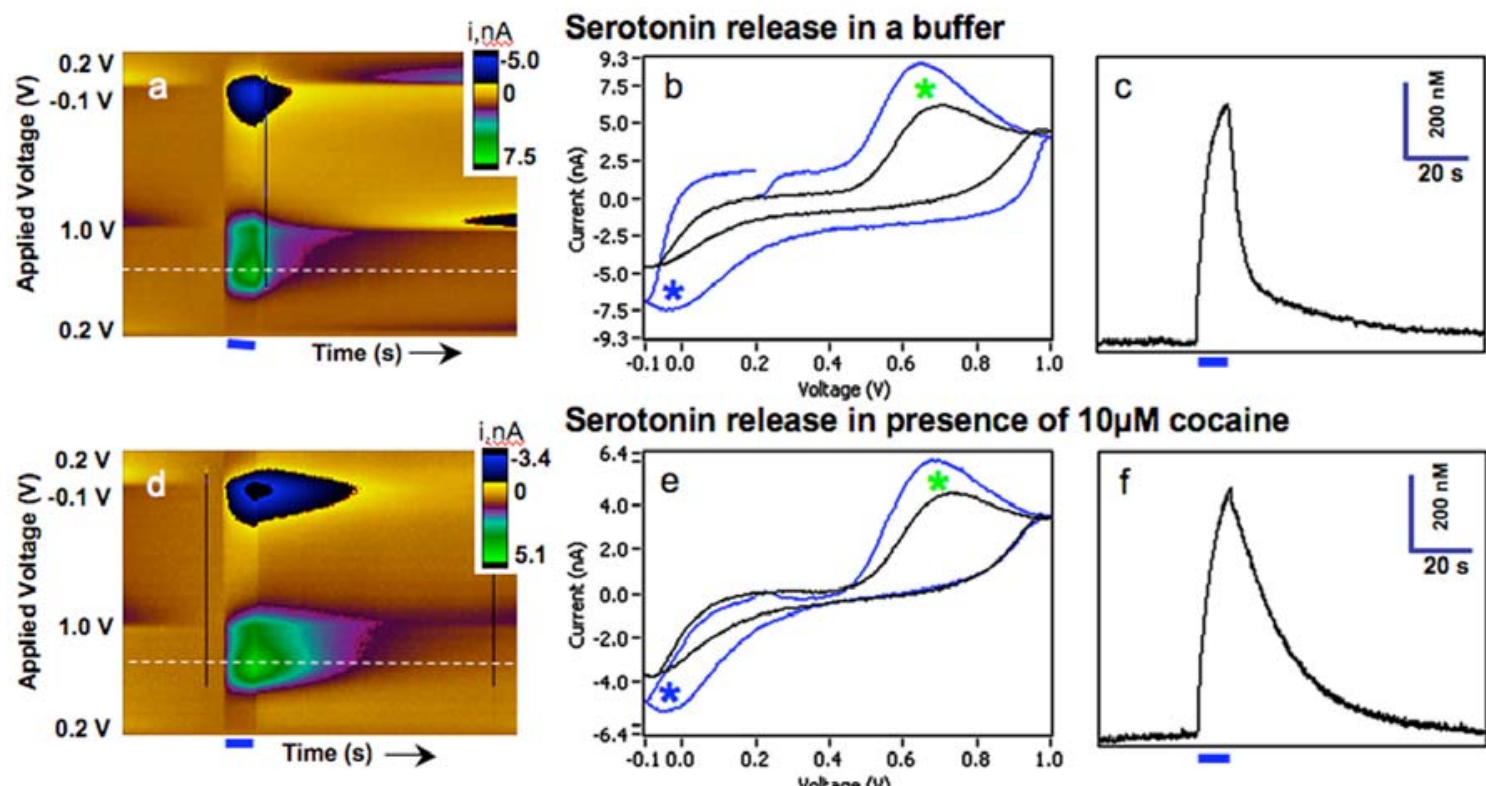

Serotonin release in presence of $10 \mu \mathrm{M}$ cocaine
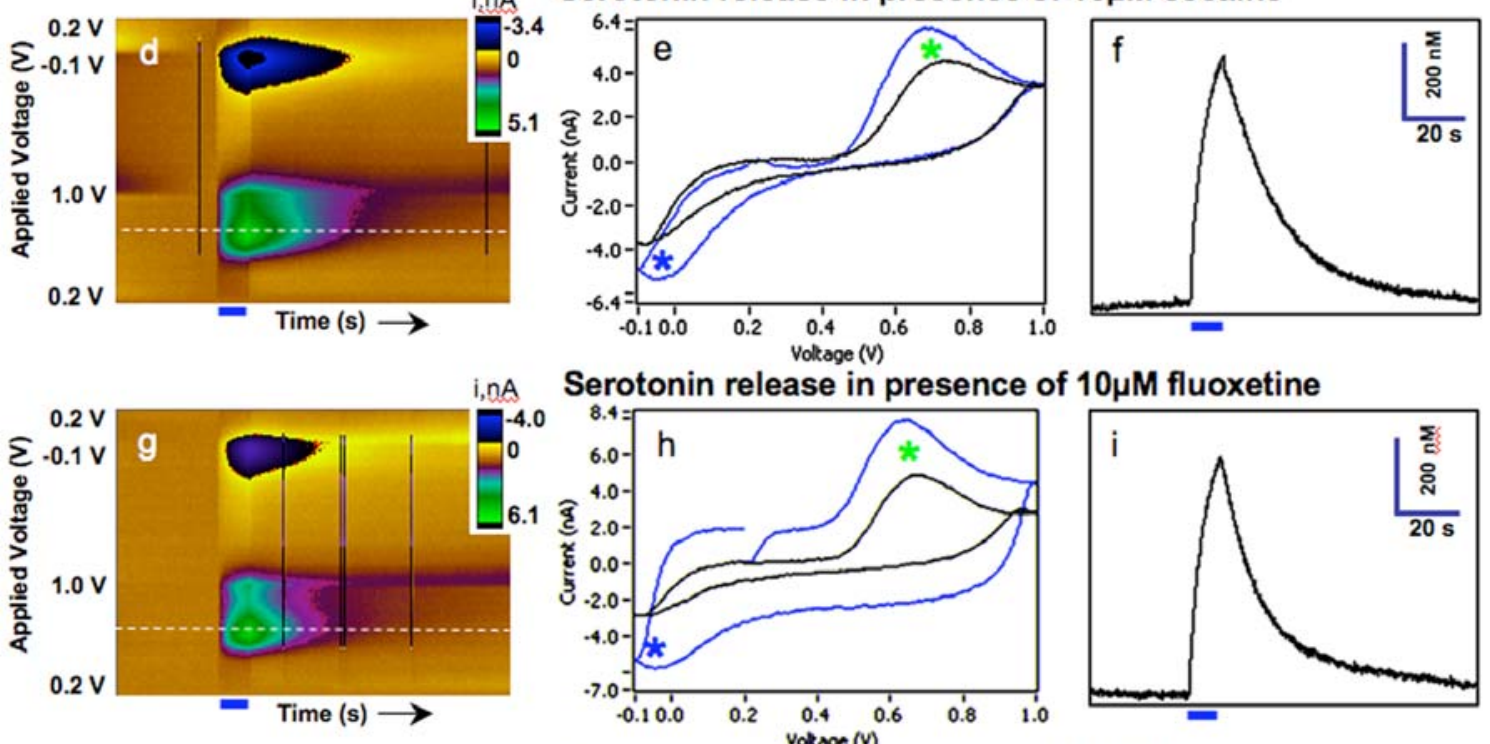

Serotonin release in presence of $10 \mu \mathrm{M}$ fluoxetine
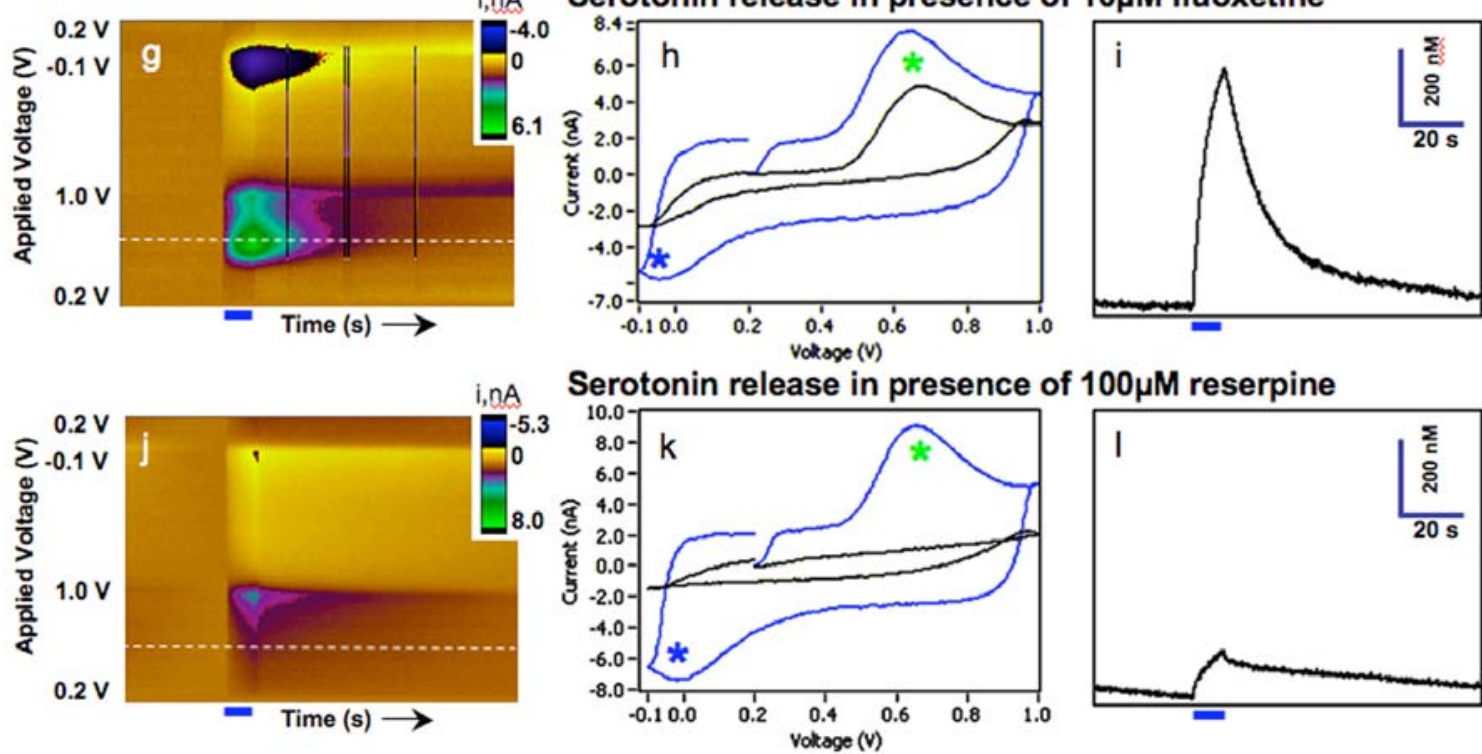

Serotonin release in presence of $100 \mu \mathrm{M}$ reserpine
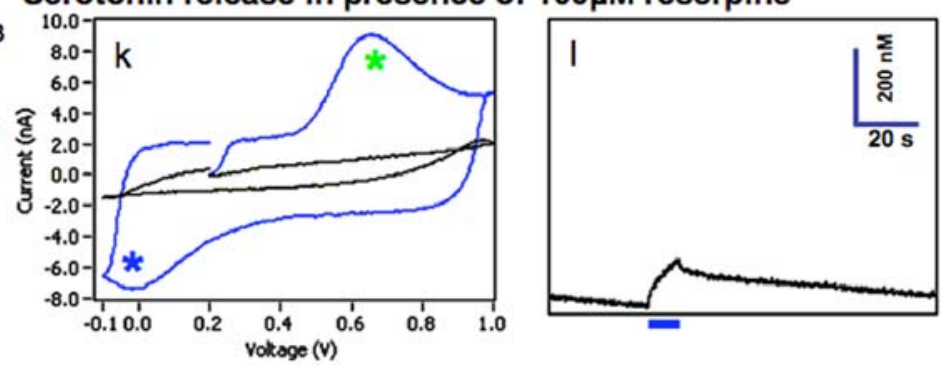

Figure 8. Pharmacological characterization of serotonin release and clearance, sample data. Duration of serotonin signal following 10 second blue light stimulation is significantly increased in VNCs exposed to cocaine or fluoxetine. These data are from the same VNCs whose signal traces were shown superimposed in fig. 5a,b. Data is shown as color plots (left column), background-subtracted CVs (middle column) with CVs from $1 \mu \mathrm{M} 5-\mathrm{HT}$ calibrations (blue lines), and signal trace taken at potential 
corresponding to dashed white line on color plots (right column). (a-c) are from a control VNC dissected in buffer. (d-f) are from a VNC incubated in cocaine, (g-i) are from a VNC incubated in fluoxetine, $(\mathrm{j}-\mathrm{I})$ are from a VNC incubated in reserpine. Note the increased duration of serotonin evident in color plots and in traces from cocaine and fluoxetine-incubated VNCs ( $d, f, g$, i). The location of oxidation and reduction peaks on all but the reserpine incubated CVs are consistent with serotonin detection. CVs from cocaine and fluoxetine-exposed VNCs (e, h, black lines) do not show the appearance of new peaks. CVs from reserpine-incubated VNCs $(k)$ do not show any peaks consistent with serotonin detection, indicating that serotonin release was not observed in these samples.

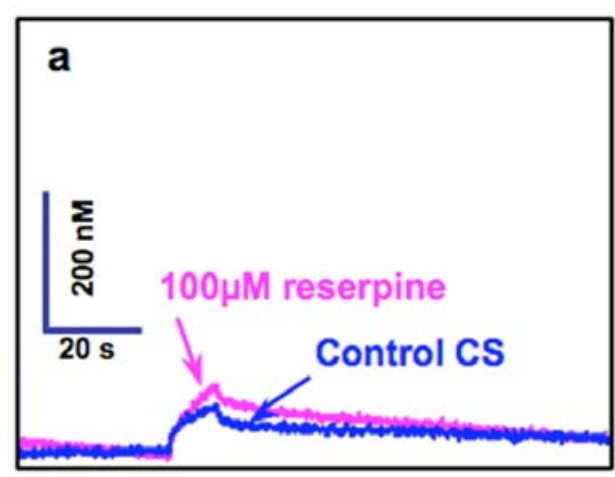

c. Peak height

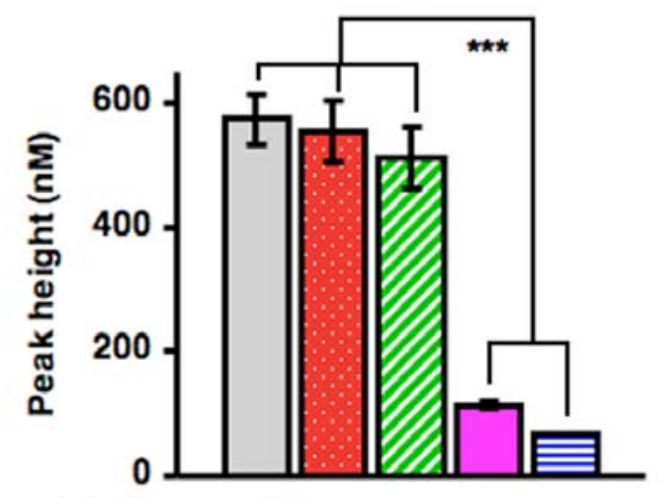

$\square$ Buffer, $\mathrm{n}=\mathbf{2 0}$

$\square 10 \mu \mathrm{M}$ Fluoxetine, $\mathrm{n}=13$

$\square$ CS control, no serotonin, $\mathrm{n}=5$

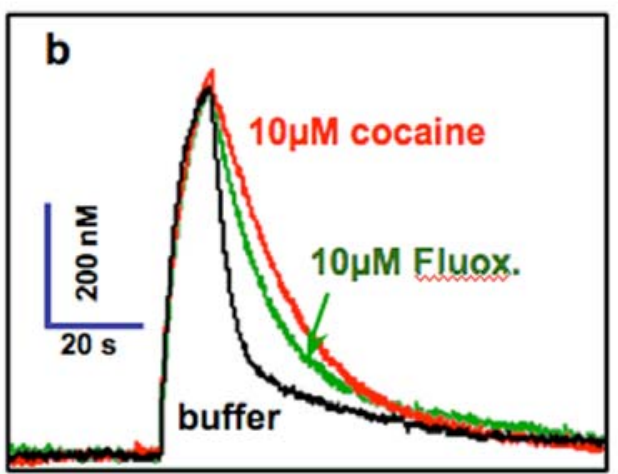

d. Serotonin clearance

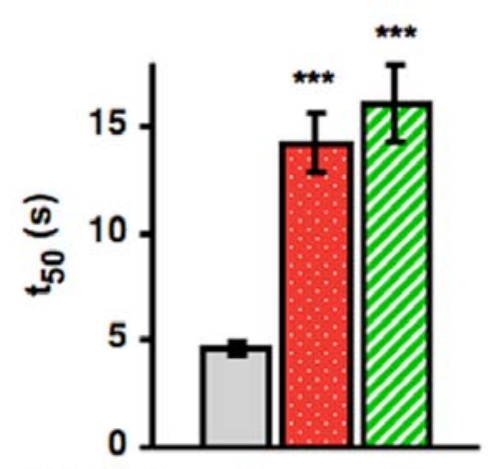

$010 \mu \mathrm{M}$ Cocaine, $\mathrm{n}=11$ $\square 100 \mu \mathrm{M}$ Reserpine, $\mathrm{n}=6$

Figure 9. Pharmacological characterization of serotonin release and clearance. VNCs were incubated in drugs for 30 minutes prior to the initiation of stimulation. (a) Representative trace from a VNC incubated in the VMAT inhibitor reserpine, 
superimposed on a trace from a control VNC that lacks ChR2 expression showing that they are similar in size and shape. (b) Representative traces from VNCs incubated in buffer, cocaine, or fluoxetine, superimposed to highlight differences in time course. (c, d) Pooled data (mean +/- SEM, $\mathrm{n}$ as shown). (c) Peak height is not affected by incubation in cocaine or fluoxetine (signal not different from buffer control group). Reserpine leads to a significant reduction in released serotonin ( $p<0.0001$, student t-test, 2 tailed), with the signal size falling below the noise threshold (signal not significantly different from control VNC). (d) Cocaine and fluoxetine-incubated VNCs exhibit significantly longer time to half maximal signal decay $(p<0.0001$, student t-test, two-tailed). Clearance measures were not performed for control or reserpine-incubated VNCs because serotonin was not detected.

\section{iv. Kinetic analysis of serotonin uptake}

To characterize serotonin clearance in Drosophila, the kinetic constants $\mathrm{K}_{\mathrm{T}}$ and $V_{\max }$ were estimated. These parameters are measures of transporter affinity and number of uptake sites, respectively. Constants were estimated using previous methods that assume all clearance is due to uptake [22-24]. Briefly, the decay portion of each peak was fit with a single exponential decay function using nonlinear regression analysis:

$$
A(t)=A_{\max }{ }^{*} e^{-k^{*} t}
$$

where $A$ is the serotonin concentration at a given time, $A_{\max }$ is peak height, and $k$ is the first-order rate constant. A representative trace superimposed with the exponential fit (yellow line on Fig. 10a) shows that the exponential decay function closely follows the actual signal. Optimal fit (maximized $R^{2}$ value) was achieved when data was fit from the time stimulation ended until $t=22-30$ s later depending on stimulation length. This time corresponded to a signal decay of about $80 \%$, the same cutoff used in previously published work [23]. 
a. Curve fitting using a single exponential decay equation

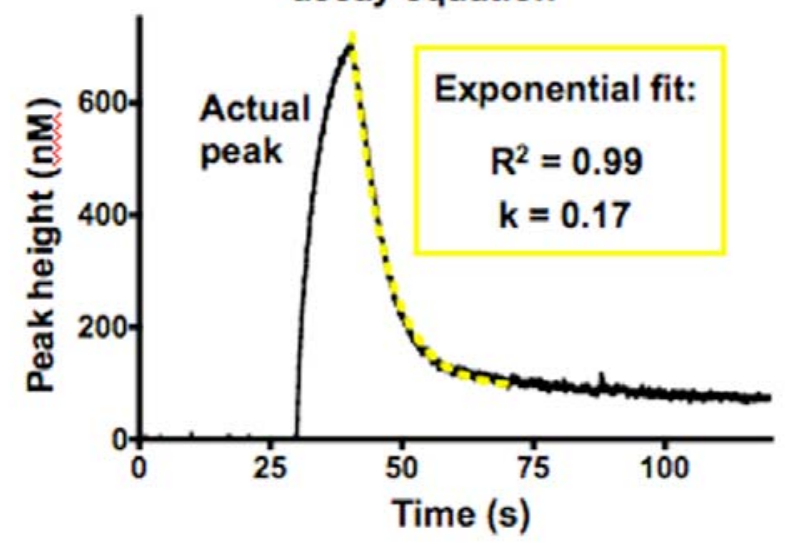

b. Velocity of serotonin clearance

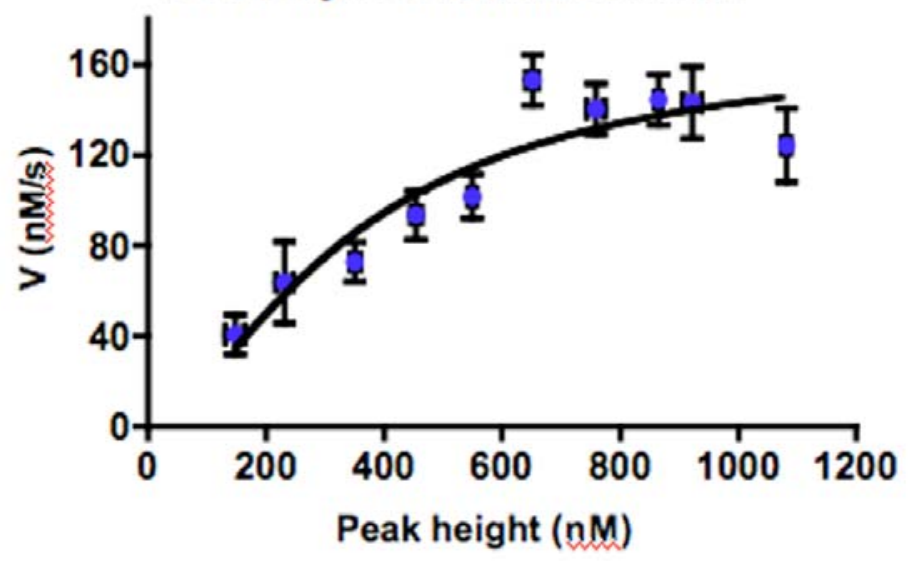

c. Efficiency of serotonin clearance
Figure 10. Kinetic parameters

governing serotonin clearance. (a) Parameters can be calculated from the elicited serotonin peaks. The decay portion of the signal is fit with a one phase exponential decay curve. From this equation, $\mathrm{k}$, the exponential constant is used to calculate an initial velocity (V). These parameters are shown (b, c) plotted against peak height (mean +/- SEM of 3-12 signals per point) (b) The initial velocity of serotonin clearance in the fly plateaus at $\sim 160 \mathrm{nM} / \mathrm{s}$. The data were fit to a 4 parameter logistics equation (line). (c) The derived clearance decay rate $(k)$ decreases as larger serotonin release is elicited.

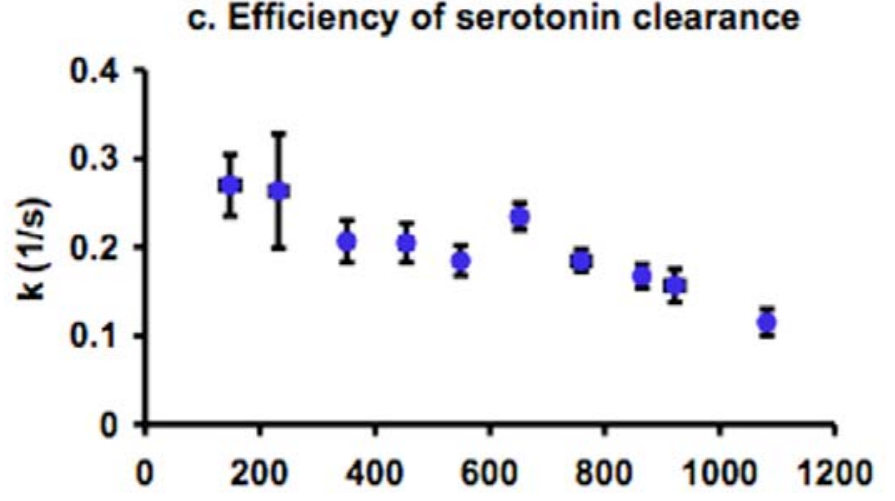

Initial velocity of serotonin clearance $(\mathrm{V})$ was calculated from the equation $\mathrm{V}=$ $k^{*} A_{\max }$. A plot of $V$ versus peak concentration (Fig. 10b) is obtained by pooling data from multiple peaks obtained during variable length stimulations (up to $10 \mathrm{~s}$ ). Data was arranged by signals of increasing peak height and then placed into bins according to a 
pseudo-geometric progression. Each point therefore has error bars in the $\mathrm{x}$ and $\mathrm{y}$ directions ( $n=3-12$ peaks per point). A four-parameter logistic equation was fit to this plot, consisting of a sigmoid function with a variable slope and the baseline constrained to zero. This equation was based on the Michaelis-Menten rate equation. To account for the fact that we base our estimation on detected as opposed to applied serotonin concentration, we left $\mathrm{H}$ unconstrained to serve as a correction factor. The equation used was $V=V_{\max }{ }^{*} A_{\max }{ }^{\wedge} H /\left(K_{T} \wedge H+A_{\max }{ }^{\wedge} H\right)$, where $K_{T}$ is the serotonin concentration corresponding to half of $V_{\max }$. From this equation, $V_{\max }$ was estimated as $170+/-40 \mathrm{nM} / \mathrm{s}$ and $K_{T}$ as $350+/-80 \mathrm{nM}$ for our data. The $R^{2}$ for this fit was 0.86 . This model predicts that a maximal rate of uptake would be achieved at concentrations greater than $4 \mu \mathrm{M}$. $\mathrm{A}$ plot of k, the exponential constant, versus evoked concentration (Fig. 10c) is obtained from the same data set. $k$ is proportional to $V_{\max } / K_{m}$ and should be constant until near $V_{\max }$, when it should decrease. At the smallest concentration we observe large errors and $\mathrm{k}$ appears high. At higher concentrations $\mathrm{k}$ is relatively stable but exhibits a slight downward trend.

When VNCs were incubated in cocaine, $k$ is significantly decreased 4.8 fold to $0.055+/-0.004(p<0.0001$, student t-test, 2 tailed, $n=11-19)$. In fluoxetine, $k$ is significantly decreased 4.4 fold to $0.06+/-0.004(p<0.0001$, student t-test, 2 tailed, $n=$ 11-15). The $k$ values derived from peaks in fluoxetine-incubated VNCs were not significantly different from the $\mathrm{k}$ values for cocaine-incubated VNCs (student t-test, 2 tailed, $p=0.4$ ).

\section{Discussion}

Knowledge of extracellular serotonin signaling in the fly has heretofore been limited by the lack of rapid detection techniques. We have optimized a novel combination of methods to induce and measure rapid changes in serotonin levels in the isolated 
ventral nerve cord of Drosophila larvae. These are the first real-time measurements of endogenous monoamine changes in Drosophila larvae. Fast-scan cyclic voltammetry at implanted carbon-fiber microelectrodes provides subsecond temporal resolution for detection of serotonin changes [15]. Expression of ChR2 allows neuronal type-specific depolarization as opposed to global stimulation typical of electrical or ionic stimulation paradigms $[19,25,26]$. When larval VNCs expressing ChR2 under the control of THgal4 or Tph-gal4 are exposed to blue light, release of endogenous dopamine or serotonin is detected, respectively. The observed concentration changes are coincident with the stimulation, therefore the ChR2-mediated depolarization is fast and neurotransmitter is released primarily during the stimulations. The CV and pharmacological agents that affect release allow us to confirm serotonin is being detected. Michaelis-Menten uptake parameters for SERT were estimated in intact Drosophila tissue for the first time.

\section{i. Serotonin release is vesicular and dependent on serotonin synthesis}

To confirm pharmacologically that the observed signal results from serotonin and not the release of another electroactive substance, VNCs were incubated with the serotonin synthesis inhibitor PCPA [27]. While VNCs dissected in buffer show relatively stable signals across repeated stimulations performed 10 minutes apart, PCPAincubated VNCs show a steady decrease in amplitude, resulting in a decrease to $47 \%$ of the initial value after 4 stimulations. The decrease in release by PCPA, in addition to the cyclic voltammogram shape, confirms that serotonin release is being measured.

Serotonin efflux predominantly occurs, in mammals and leech, through vesicle exocytosis and not through reverse transport by SERT [28-30]. Our work shows that release in the fly is also vesicular because it is dependent on the packaging of serotonin into secretory vesicles by VMAT. When VMAT is inhibited by reserpine, serotonin 
release is not observed. The rate at which serotonin release capacity decays in the presence of reserpine is rapid, with complete elimination of the serotonin signal observed within 30 minutes of drug exposure. This is different from the pattern of serotonin release in the presence of PCPA, where the initial peak was not significantly different from that in buffer. When only synthesis is inhibited, recycling of serotonin by the transporter may be able to maintain a vesicular population. The severe serotonin depletion observed after reserpine incubation may also be due to non-specific release of vesicular serotonin. However, this proposed mechanism of reserpine action is not well characterized and it is unclear if it occurs in the fly [31, 32].

\section{ii. Characterization of serotonin release in the fly}

Channelrhodopsin2-mediated stimulation elicits release of endogenous serotonin in physiologically relevant concentrations. Because serotonin release has not been previously characterized in the fly, we performed calculations to estimate the number of vesicles needed to produce extracellular serotonin concentrations similar to those measured. We assumed that each release site would synchronously release one vesicle into the extracellular space and that reuptake would not decrease the size of the resulting peak. The wandering third instar Drosophila VNC neuropil contains an average of 12 varicosities per $1,000 \mu \mathrm{m}^{3}$, which are putative serotonin release sites [33]. If every varicosity released one vesicle containing 4,700 molecules of serotonin [34], the resulting concentration in the sampling volume would equal $450 \mathrm{nM}$. We detected serotonin concentrations ranging from 280 to $640 \mathrm{nM}$ following $2 \mathrm{~s}$ to 30 s of blue light exposure. These values are consistent with those obtained by electrical stimulation in mammalian substantia nigra reticulata $(\mathrm{SNr})$ slices, where values ranging from 54 to 640 $\mathrm{nM}$ were detected for 1 to 20 electrical pulses [11]. The detected concentrations range from $64 \%$ to $142 \%$ of the concentration expected from synchronous single quantum 
release in the fly. Therefore, on average, during maximal stimulation each varicosity releases 1.5 vesicles.

While the above calculation shows that the values obtained after blue light stimulation are within the physiologically-relevant range, it does not allow us to make any inferences about the release at specific varicosities. The electrode, because of its size, samples from many varicosities [35]. Some varicosities may not be stimulated, so the actual amount released per activated varicosity may be greater. Additionally, reuptake acts to decrease the observed peak concentration, likely causing an underestimation of actual release [11]. This error would be most severe for 30 s long stimulations and this balance of release and reuptake would explain why the peak shape plateaus during the stimulation.

\section{iii. Effect of transporter inhibition on serotonin clearance}

We expect that released serotonin is cleared primarily through reuptake. To test this hypothesis, we blocked transporter function with cocaine or fluoxetine. Both drugs lengthened the time for serotonin to decay back to baseline, an indication that uptake is involved in serotonin clearance. In humans, cocaine shows nearly equal affinity for SERT and DAT, while in the fly cocaine has $6 x$ greater affinity for SERT (dSERT $K_{i}=$ $0.47 \mu \mathrm{M}$, versus dDAT $K_{i}=2.7 \mu \mathrm{M}$ [36]) [37]. Fluoxetine is a typical selective serotonin reuptake inhibitor in humans, but it has a more limited selectivity in Drosophila (dSERT $\mathrm{K}_{\mathrm{i}}=73 \mathrm{nM}$ vs $\mathrm{dDAT} \mathrm{K}_{\mathrm{i}}=240 \mathrm{nM}$ [36]). Although serotonin can be taken up by the dopamine transporter, the published $\mathrm{K}_{\mathrm{m}}$ of dDAT for serotonin $(43 \mu \mathrm{M})$ is 86 times larger than that of dSERT $(490-637 \mathrm{nM})[36,37]$. The peak concentrations observed in these experiments were $280-640 \mathrm{nM}$. This is very close to the $K_{m}$ for SERT but over $67 x$ less than that for DAT. Assuming that SERT and DAT transport serotonin at similar rates, at the observed concentrations, DAT would transport an order of magnitude less serotonin 
than SERT. It is therefore unlikely that DAT-mediated reuptake makes a sizeable contribution to the clearance of serotonin.

\section{iv. Kinetic analysis of serotonin uptake in the fly}

Michaelis-Menten modeling can be used to describe parameters for uptake. $\mathrm{V}_{\max }$ is the maximal rate of uptake, which is dependent on the number of transporters. $\mathrm{K}_{\mathrm{T}}$ is the serotonin concentration at which the clearance rate is half maximal. These are the first estimates of uptake parameters in intact tissue in Drosophila. Our estimated $\mathrm{K}_{\mathrm{T}}$ for serotonin clearance in the fly is $350+/-80 \mathrm{nM}$. $\mathrm{K}_{\mathrm{T}}$ should be of the same order of magnitude as $K_{m}$ and this is close to previously published $K_{m}$ values of 490 and $637 \mathrm{nM}$ for dSERT obtained from studies using transfected cells [36, 37]. $\mathrm{K}_{\mathrm{m}}$ measurements in mammals have ranged from $170 \mathrm{nM}[11]$ to $5.4 \mu \mathrm{M}$ [22]. The estimated $\mathrm{V}_{\max }$ is $170+/-$ $40 \mathrm{nM} / \mathrm{s}$, similar to the $198 \mathrm{nM} / \mathrm{s}$ observed for mammalian SERT in the hippocampus [22]. While our kinetic parameters are just estimates, as the elicited peak heights are smaller than the concentration necessary to produce $V_{\max }$, they show that the parameters in the fly are of similar to those in mammals. Future studies could use the application of exogenous serotonin to produce larger serotonin concentrations and provide a better estimate of $\mathrm{V}_{\max }$. A caveat to these calculations, including the previously published values, is that they do not take diffusion into account. While the values derived here can be compared to similarly-derived values, they may overestimate the actual $V_{\max }$ for dSERT because all clearance is assumed to be due to uptake. The role of diffusion in the clearance of serotonin could be investigated by monitoring the clearance of a similarly-sized but non-transported molecule and represents a key area for future research.

Acute transporter inhibition results in a significant increase in $t_{50}$ with no significant effect on serotonin release. This finding is consistent with a key role of 
reuptake in the clearance of serotonin and with results from electrical stimulation in brain slices [11]. When transporter activity is inhibited with cocaine or fluoxetine, $\mathrm{k}$ is decreased 4.8 fold or 4.4 fold respectively. The $k$ values for fluoxetine were not significantly different from cocaine indicating that the drugs had a similar effect on serotonin clearance. Assuming these are competitive uptake inhibitors and that $\mathrm{V}_{\max }$ remains the same, a change in $\mathrm{k}$ would be inversely proportional to a change in $\mathrm{K}_{\mathrm{m}}$. Therefore, both drugs increase apparent $\mathrm{K}_{\mathrm{m}}$ over 4-fold. These changes are of similar magnitude to the 5-6 fold changes in $K_{m}$ that have been observed after cocaine or fluoxetine incubation in mammalian substantia nigra slices [24]. Serotonin release in the fly is localized to a small region in the interior of the CNS, the neuropil, with the majority of cell bodies residing outside this area. With this morphology, diffusion may play a larger role in clearing serotonin released in the neuropil and therefore less difference may be observed after the inhibition of transport.

\section{Conclusions}

Monoamine changes have been routinely measured in mammals [10]. However, powerful genetic tools are available for Drosophila which, combined with our technique, would allow large scale genetic analysis of neurotransmitter dynamics. For example, human polymorphisms affecting SERT expression are associated with depression, anxiety, and efficacy of antidepressants [1]. Genetic screens in the fly could help identify genetic elements critical for SERT regulation of serotonin. While this study focused on characterizing serotonin concentrations, we also demonstrated the use of our technique to monitor dopamine. Extension of this technique to encompass other neuronal types is possible because FSCV can detect other electroactive neurotransmitters and enzymebased biosensors could be used for other neurotransmitters. This study shows that the dynamics of serotonin release and reuptake in Drosophila are analogous to those in 
mammals, making this simple organism more useful for studying the basic physiological mechanisms of neurotransmission. 


\section{References}

1. Murphy, D.L., A. Lerner, G. Rudnick, and K.P. Lesch, Serotonin transporter: gene, genetic disorders, and pharmacogenetics. Mol Interv, 2004. 4(2): p. 109-23.

2. Serretti, A., M. Kato, D. De Ronchi, and T. Kinoshita, Meta-analysis of serotonin transporter gene promoter polymorphism (5-HTTLPR) association with selective serotonin reuptake inhibitor efficacy in depressed patients. Mol Psychiatry, 2006. 12(3): p. 247-257.

3. Lasky-Su, J.A., S.V. Faraone, S.J. Glatt, and M.T. Tsuang, Meta-analysis of the association between two polymorphisms in the serotonin transporter gene and affective disorders. Am J Med Genet B Neuropsychiatr Genet, 2005. 133(1): p. $110-5$.

4. Nichols, C.D., 5-HT2 receptors in Drosophila are expressed in the brain and modulate aspects of circadian behaviors. Dev Neurobiol, 2007. 67(6): p. 752-63.

5. Bier, E., Drosophila, the golden bug, emerges as a tool for human genetics. Nat Rev Genet, 2005. 6(1): p. 9-23.

6. Yuan, Q., F. Lin, X. Zheng, and A. Sehgal, Serotonin modulates circadian entrainment in Drosophila. Neuron, 2005. 47(1): p. 115-27.

7. Sykes, P.A. and B.G. Condron, Development and sensitivity to serotonin of Drosophila serotonergic varicosities in the central nervous system. Dev Biol, 2005. 286(1): p. 207-16.

8. $\quad$ Park, S.K., et al., Cell-type-specific limitation on in vivo serotonin storage following ectopic expression of the Drosophila serotonin transporter, dSERT. Journal of Neurobiology, 2006. 66(5): p. 452-462.

9. Paxon, T.L., P.R. Powell, H.G. Lee, K.A. Han, and A.G. Ewing, Microcolumn separation of amine metabolites in the fruit fly. Anal Chem, 2005. 77(16): p. 5349-55.

10. Phillips, P.E.M., G.D. Stuber, M.L.A.V. Heien, R.M. Wightman, and R.M. Carelli, Subsecond dopamine release promotes cocaine seeking. Nature, 2003. 422(6932): p. 614-618.

11. Bunin, M.A. and R.M. Wightman, Quantitative evaluation of 5hydroxytryptamine (serotonin) neuronal release and uptake: an investigation of extrasynaptic transmission. J Neurosci, 1998. 18(13): p. 4854-60.

12. Garris, P.A., et al., A role for presynaptic mechanisms in the actions of nomifensine and haloperidol. Neuroscience, 2003. 118(3): p. 819-829.

13. Venton, B.J., et al., Cocaine Increases Dopamine Release by Mobilization of a Synapsin-Dependent Reserve Pool. Journal of Neuroscience, 2006. 26(12): p. 3206-3209.

14. Heien, M.L.A.V., P.E.M. Phillips, G.D. Stuber, A.T. Seipel, and R.M. Wightman, Overoxidation of carbon-fiber microelectrodes enhances dopamine adsorption and increases sensitivity. The Analyst, 2003. 128(12): p. 1413-1419.

15. Jackson, B.P., S.M. Dietz, and R.M. Wightman, Fast-scan cyclic voltammetry of 5-hydroxytryptamine. Anal Chem, 1995. 67(6): p. 1115-20. 
16. Davidson, C., E.H. Ellinwood, S.B. Douglas, and T.H. Lee, Effect of cocaine, nomifensine, GBR 12909 and WIN 35428 on carbon fiber microelectrode sensitivity for voltammetric recording of dopamine. J Neurosci Methods, 2000. 101(1): p. 75-83.

17. Swamy, B.E. and B.J. Venton, Subsecond detection of physiological adenosine concentrations using fast-scan cyclic voltammetry. Anal Chem, 2007. 79(2): p. 744-50.

18. Heien, M.L., M.A. Johnson, and R.M. Wightman, Resolving Neurotransmitters Detected by Fast-Scan Cyclic Voltammetry. Anal. Chem., 2004. 76(19): p. 56975704.

19. Schroll, C., et al., Light-induced activation of distinct modulatory neurons triggers appetitive or aversive learning in Drosophila larvae. Curr Biol, 2006. 16(17): p. 1741-7.

20. Zhang, X., J.-M. Beaulieu, T.D. Sotnikova, R.R. Gainetdinov, and M.G. Caron, Tryptophan Hydroxylase-2 Controls Brain Serotonin Synthesis. Science, 2004. 305(5681): p. 217.

21. Zhou, F.M., Y. Liang, R. Salas, L. Zhang, M. De Biasi, and J.A. Dani, Corelease of dopamine and serotonin from striatal dopamine terminals. Neuron, 2005. 46(1): p. 65-74.

22. Daws, L.C., S. Montanez, W.A. Owens, G.G. Gould, A. Frazer, G.M. Toney, and G.A. Gerhardt, Transport mechanisms governing serotonin clearance in vivo revealed by high-speed chronoamperometry. J Neurosci Methods, 2005. 143(1): p. 49-62.

23. Sabeti, J., C.E. Adams, J. Burmeister, G.A. Gerhardt, and N.R. Zahniser, Kinetic analysis of striatal clearance of exogenous dopamine recorded by chronoamperometry in freely-moving rats. J Neurosci Methods, 2002. 121(1): p. 41-52.

24. John, C.E. and S.R. Jones, Voltammetric characterization of the effect of monoamine uptake inhibitors and releasers on dopamine and serotonin uptake in mouse caudate-putamen and substantia nigra slices. Neuropharmacology, 2007. 52(8): p. 1596-605.

25. Parrish, A.R., W. Wang, and L. Wang, Manipulating proteins for neuroscience. Curr Opin Neurobiol, 2006. 16(5): p. 585-92.

26. Zhang, W., W. Ge, and Z. Wang, A toolbox for light control of Drosophila behaviors through Channelrhodopsin 2-mediated photoactivation of targeted neurons. Eur J Neurosci, 2007. 26(9): p. 2405-16.

27. Dasari, S., K. Viele, A.C. Turner, and R.L. Cooper, Influence of PCPA and MDMA (ecstasy) on physiology, development and behavior in Drosophila melanogaster. Eur J Neurosci, 2007. 26(2): p. 424-38.

28. Fon, E.A., E.N. Pothos, B.-C. Sun, N. Killeen, D. Sulzer, and R.H. Edwards, Vesicular Transport Regulates Monoamine Storage and Release but Is Not Essential for Amphetamine Action. Neuron, 1997. 19(6): p. 1271-1283.

29. Wang, Y.M., et al., Knockout of the vesicular monoamine transporter 2 gene results in neonatal death and supersensitivity to cocaine and amphetamine. Neuron, 1997. 19(6): p. 1285-96. 
30. Bruns, D., D. Riedel, J. Klingauf, and R. Jahn, Quantal release of serotonin. Neuron, 2000. 28(1): p. 205-20.

31. Pletscher, A., P.A. Shore, and B.B. Brodie, Serotonin release as a possible mechanism of reserpine action. Science, 1955. 122(3165): p. 374-5.

32. Reimann, W. and F. Schneider, Induction of 5-hydroxytryptamine release by tramadol, fenfluramine and reserpine. Eur J Pharmacol, 1998. 349(2-3): p. 199203.

33. Chen, J. and B.G. Condron, Branch architecture of the fly larval abdominal serotonergic neurons. Dev Biol, 2008. 320(1): p. 30-8.

34. Bruns, D. and R. Jahn, Real-time measurement of transmitter release from single synaptic vesicles. Nature, 1995. 377(6544): p. 62-65.

35. Wightman, R.M., Detection technologies. Probing cellular chemistry in biological systems with microelectrodes. Science, 2006. 311(5767): p. 1570-4.

36. Porzgen, P., S.K. Park, J. Hirsh, M.S. Sonders, and S.G. Amara, The Antidepressant-Sensitive Dopamine Transporter in Drosophila melanogaster: A Primordial Carrier for Catecholamines. Molecular Pharmacology, 2001. 59(1): p. 83-95.

37. Corey, J.L., M.W. Quick, N. Davidson, H.A. Lester, and J. Guastella, A cocainesensitive Drosophila serotonin transporter: cloning, expression, and electrophysiological characterization. Proc Natl Acad Sci U S A, 1994. 91(3): p. 1188-92. 


\title{
Chapter 4. Serotonin synthesis and reuptake work synergistically to maintain the neuronal serotonin pool in Drosophila.
}

\author{
Abstract \\ Selective serotonin reuptake inhibitors (SSRIs) have been the standard of \\ treatment for several mental disorders, including major depressive disorder. However, \\ our understanding of their effects is incomplete and the serotonergic system in mammals \\ is complex. We have developed a novel combination of methods to study serotonergic \\ signaling in the larval Drosophila central nervous system. Fast-scan cyclic voltammetry \\ at implanted microelectrodes is used to detect serotonin elicited by channelrhodopsin-2- \\ mediated depolarization. The effects of pharmacologically inhibiting serotonin synthesis \\ or reuptake on evoked serotonin were probed by incubating in cocaine or $p$ - \\ chloroalanine (PCPA), respectively. Stimulations were repeated at various intervals to \\ assess the time course of recovery of the releasable pool. Reuptake is important for the \\ clearance of serotonin as well as the rapid replenishment of the releasable pool. \\ Synthesis is critical to the longer-term replenishment of the releasable pool, especially \\ when reuptake is concurrently inhibited. Within 30 minutes of synthesis and reuptake \\ inhibition, neuronal serotonin content decreased by $50 \%$ and the initial amount of \\ evoked serotonin is reduced by $65 \%$. Decreases in serotonin are rescued by inhibiting \\ action potential propagation with tetrodotoxin, implicating endogenous activity in the \\ depletion of neuronal serotonin. These results show a direct link between releasable \\ serotonin, serotonin synthesis, and reuptake, suggesting that synthesis may be \\ important for the clinical effects of SSRIs.
}




\section{Introduction}

The serotonin transporter (SERT) plays a key role in regulating extracellular serotonin levels and polymorphisms conferring decreased activity have been associated with anxiety and the development of depression under stressful conditions [1-3]. Selective serotonin reuptake inhibitors (SSRIs), are used to treat mental illness, particularly monopolar depression, which is diagnosed in approximately $5-10 \%$ of Americans per year [4]. However, the time between treatment onset and effect is delayed and 30-40\% of people with depression do not respond to SSRI treatment [5]. A better understanding of the physiology of serotonergic neurons that contributes to this effect would aid in the development and treatment of depression.

In the central nervous system, serotonin is synthesized by a two-step enzymatic process beginning with the rate-limiting enzyme, tryptophan hydroxylase 2 (Tph2), which converts tryptophan into 5-hydroxytryptophan [6]. After packaging into vesicles by the vesicular monoamine transporter (VMAT), serotonin is released via exocytosis into the extracellular space. Serotonergic neurons are spontaneously active at 0.5 to $1 \mathrm{~Hz}$ in the invertebrate lobster [7] and $0.6 \mathrm{~Hz}$ in mammals, with a subpopulation undergoing burst firing [8]. Reuptake by SERT is the main mechanism that stops extracellular serotonergic signaling. Although the role of reuptake has been heavily investigated, the role of serotonin synthesis in serotonergic signaling and in mental illnesses is not well understood. Tph2 polymorphisms conferring decreased expression have been associated with suicidal behavior [3], but this data and its interpretation remain controversial [9].

The complexity of the serotonergic system in mammals makes it difficult to study and Drosophila provides an attractive alternative [10]. The fly boasts a fully developed network of serotonergic neurons within a small, easily accessible and relatively simple central nervous system $[11,12]$. Until recently, measurements of real-time serotonin 
release and reuptake in the fly have been hampered by a lack of analytical tools. We have developed a method using channelrhodopsin-2 (ChR2) mediated stimulation to elicit the release of endogenous serotonin, which is detected in real time using fast scan cyclic voltammetry (FSCV) at implanted microelectrodes [13]. Stimulating release allows measurements of serotonin available for exocytosis, the releasable pool.

In this study, we evaluate the roles of synthesis and reuptake in maintaining the size of the releasable pool. We use repeated stimulations and pharmacological manipulations to show that in the fly, reuptake is necessary for the short-term replenishment of the releasable pool while synthesis is more important over longer periods. Work in mice previously suggested that serotonin availability might be important for the effects of SSRIs [14]. In Drosophila, when reuptake is concurrently inhibited, synthesis is critical to replenishing serotonin released during endogenous or stimulated release. This work shows that synthesis influences the effects of SSRIs, thereby expanding our understanding of the physiological effects of SSRIs on serotonergic neurons.

\section{Materials and Methods}

i. Fly stocks

Flies containing Channelrhodopsin-2 (ChR2, a gift from Christian Schroll, Universitat Wurzburg) were crossed to flies expressing Tph-GAL4 (a gift from Jaeson Kim, Korea Advanced Institute of Science and Technology) to generate a homozygous line. Larvae were allowed to feed on yeast supplemented with $10 \mathrm{mM}$ all-trans retinal (Sigma-Aldrich, St. Louis) for 2-3 days prior to the initiation of experiments while protected from light. 


\section{ii. Immunohistochemistry}

To estimate neuronal serotonin levels, we performed immunohistochemistry for the neurotransmitter. Larval ventral nerve cords (VNCs) were dissected and imaged as previously described [15]. Briefly, VNCs were incubated in buffer for 30 minutes prior to fixation with $4 \%$ paraformaldehyde for $1 \mathrm{~h}$ at room temperature. VNCs were then incubated in phosphate buffered saline with $1 \%$ tween (PBT) overnight at room temperature with 1:666 anti-serotonin (rabbit polyclonal, ImmunoStar). Alexafluor goat anti-rabbit 568 (Molecular Probes) secondary antibody was subsequently applied at 1:1000 in PBT overnight at room temperature. VNCs were mounted to slides in $90 \%$ glycerol/2.5\% 1,4-diazabicyclo[2.2.2]octane and allowed to clear overnight prior to imaging under a confocal microscope.

Neuronal serotonin content was quantified by importing images of stained neuropil into imageJ and measuring mean gray levels in a rectangle that covers the middle $2 / 3$ of the neuropil region in the image. Neuronal cell bodies were included in the analysis. This area was chosen because it showed the least amount of staining variability between VNCs. Because this area also tended to experience the smallest reductions in staining, this approach may somewhat minimize the impact of drug treatments. VNCs incubated in drugs were dissected and processed at the same time as control VNCs dissected in buffer. Mean gray levels (MGL) were normalized as a percent of MGL in accompanying buffer controls to account for small variations in overall staining intensity.

\section{iii. Instrumentation and electrochemistry}

Carbon-fiber microelectrodes were manufactured from single T-650 carbon fibers, $7 \mu \mathrm{m}$ in diameter (Cytec Engineering Materials, West Patterson, NJ), as previously described [16]. A Dagan ChemClamp potentiostat was used to collect 
electrochemistry data (Dagan, Minneapolis, MN; custom-modified). Data acquisition software and hardware were the same as described by Heien et al. [17]. To detect serotonin, the voltage was ramped from $0.2 \mathrm{~V}$ to $1.0 \mathrm{~V}$, then to $-0.1 \mathrm{~V}$ and back to $0.2 \mathrm{~V}$ at a slew rate of $1,000 \mathrm{~V} / \mathrm{s}$ and a repetition rate of $10 \mathrm{~Hz}$ [18]. A silver-silver chloride wire was used as a reference electrode. Electrodes were calibrated with $1 \mu \mathrm{M}$ serotonin prior to and after use in situ.

iv. Preparation of ventral nerve cords (VNCs) and data collection

VNC preparation and data collection were performed as described in [13]. Briefly, 5 day old larval brains were dissected in modified Schneider's insect media (15.2 mM $\mathrm{MgSO}_{4}, 21 \mathrm{mM} \mathrm{KCl}, 3.3 \mathrm{mM} \mathrm{KH}_{2} \mathrm{PO} 4,53 \mathrm{mM} \mathrm{NaCl}, 5.8 \mathrm{mM} \mathrm{NaH}_{2} \mathrm{PO}_{4}, 5.4 \mathrm{mM} \mathrm{CaCl}_{2}$, $11.1 \mathrm{mM}$ Glucose, $5.3 \mathrm{mM}$ Trehalose, $\mathrm{pH}=6.2$ ). 4-Chloro-DL-phenylalanine (PCPA) and cocaine hydrochloride were purchased from Sigma-Aldrich. Tetrodotoxin (TTX) was obtained from Alomone Labs (Stock \#T-550) and resuspended in $\mathrm{dd}_{2} \mathrm{O}$ to obtain a 1 $\mathrm{mM}$ stock concentration. Frozen aliquots were diluted to the working concentration $(0.5$ $\mu \mathrm{M})$ in buffer at the time of the experiment. VNCs were allowed to incubate in buffer or drug for 25-30 min prior to the initiation of experiments to allow for drug diffusion. An electrode was implanted in the neuropil using a micromanipulator and allowed to equilibrate for $5 \mathrm{~min}$ before the experiment was initiated. After the collection of at least 30 seconds of baseline data, VNCs were exposed to 10 seconds of intense blue light to stimulate release. The stimulation length was previously determined to be the shortest exposure producing close to maximal serotonin release [13]. The light source was a 10W halogen microscope bulb with a standard fluorescein excitation filter (450 - $490 \mathrm{~nm}$ ) that was manually-switched. 


\section{v. Data analysis}

Electrochemical data was analyzed using Tar Heel CV software [19]. Data were visualized as a background-subtracted cyclic voltammogram (CV) or a concentration vs. time trace. For a detailed description of serotonin detection in the fly using FSCV, see Borue [13]. Cyclic voltammograms were used to verify serotonin was detected and identify the voltage corresponding to the maximum serotonin oxidation. The current at this voltage was converted to serotonin concentration using post calibration data for each electrode, and these changes in concentration were plotted as the signal concentration vs time trace. The height of the initial peak was used to normalize data for multiple stimulation assays. For these assays, stimulations were performed with the start of the 10 s stimulation occurring every $1,2,5$, or 10 minutes.

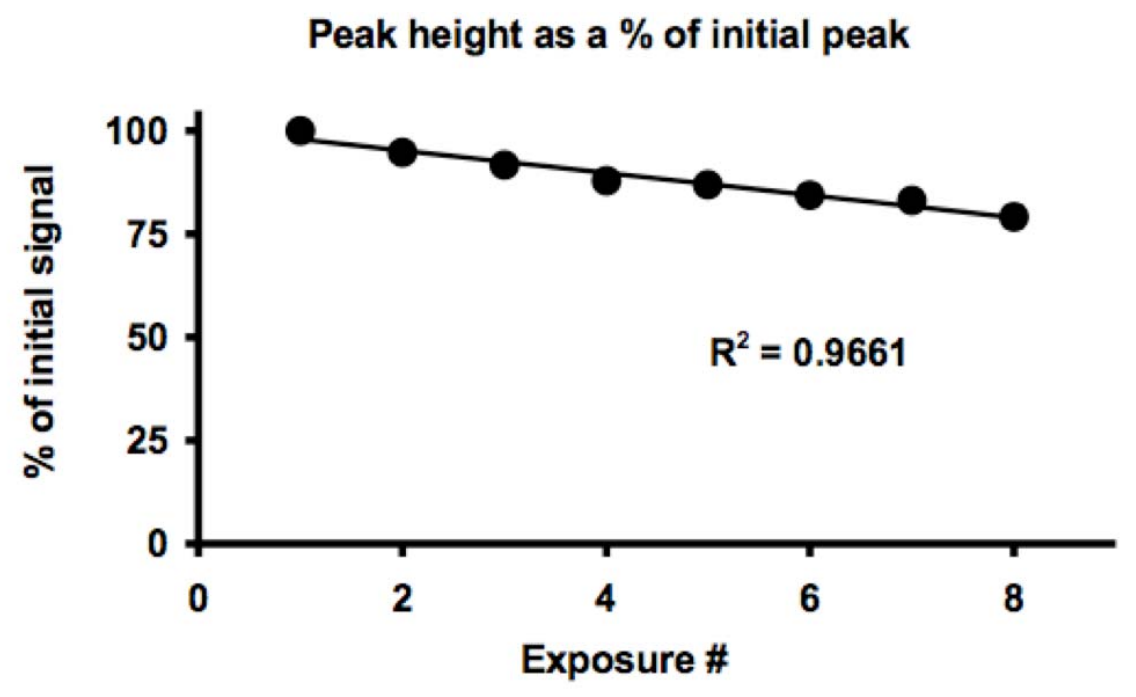

Figure 1. To determine what effect repeat serotonin exposure has on electrode sensitivity to serotonin, electrodes were exposed to serotonin in a flow cell. Electrodes were exposed to $500 \mathrm{nM}$ serotonin for 10 s every minute. Readings were normalized to the size of the pre-drug signal for each electrode. The pooled results, mean +/- SEM, for 5 electrodes are shown above. The signal appears to decrease linearly. The fit line (with an $R^{2}$ of 0.9661 ) was used to generate a correction coefficient for this decrease in serotonin sensitivity across repeated serotonin exposures. 
A series of in vitro experiments performed in a flow cell showed that electrode sensitivity to serotonin progressively decreased over the course of multiple exposures (Figure 1). A linear correction coefficient was applied to all data from multiple stimulation experiments to correct for changes in electrode sensitivity. This correction does not change the interpretation of data because it does not change the relationships between categories of VNCs. Statistical analysis of pooled data including two-tailed student t tests was conducted using Excel software. One and two way ANOVA analysis was performed using GraphPad Prism software (San Diego, CA).

\section{Results}

\section{i. Neuronal serotonin content after pharmacological manipulations}

We explored the effects of pharmacological manipulations on neuronal serotonin content as measured by immunohistochemistry. Our hypothesis was that pharmacological inhibition of uptake or release would decrease neuronal serotonin levels because both are necessary to maintain the releasable serotonin pool. We also hypothesized that endogenous neuronal firing would lead to depletion of neuronal serotonin levels. Immunohistochemistry offers very high spatial resolution, which allows an examination of the tissue morphology and serotonin localization. However, it provides only an estimate of neuronal serotonin levels due to antibody-mediated amplification of fixed serotonin. Nevertheless, these rough numbers can be used to develop hypotheses about the relative importance of each of the mechanisms and are useful for comparison with the chemical data measured with microelectrodes.

Ventral nerve cords (VNCs) from mature, 5 day old, larval Drosophila were dissected and allowed to incubate for 30 minutes in buffer or drugs, then fixed and stained for serotonin. Composite images derived from confocal stacks through the neuropil of each VNC are shown in Figure 2. To provide an objective measure of 
staining intensity, mean gray levels (MGLs) were measured in a rectangle covering the middle two-thirds of the VNC because it showed the least amount of staining variability between VNCs. MGLs for experimental VNCs were normalized to those from controls stained and imaged at the same time to account for minor differences in overall staining intensities.

A control VNC incubated in buffer shows normal serotonergic morphology and serotonin content (Fig. 2a). The VNC is a segmented structure that contains four serotonergic neurons per segment, two on each side [15]. The serotonergic cell bodies are visible as rows of brightly stained pairs on either side of the neuropil, which contains the axonal projections of the serotonergic neurons. These projections fill the neuropil region and appear as two strips of brightly stained material overlying the cell bodies. The imaging was optimized to show residual serotonin staining in drug-exposed VNCs and therefore the control VNC appears slightly over-exposed.

Since serotonergic neurons are thought to be spontaneously active, we investigated the impact of inhibiting action potential propagation on serotonin levels by using tetrodotoxin (TTX). A representative image from a VNC incubated in $0.5 \mu \mathrm{M}$ TTX shows similar staining brightness to the buffer control (Fig. 2b). Pooled data from $8-11$ samples per category (Fig. 2i) indicate that serotonin levels in VNCs incubated in TTX were not significantly different from those incubated in buffer (Student t-test, 2-tailed, $p=$ $0.25)$. 

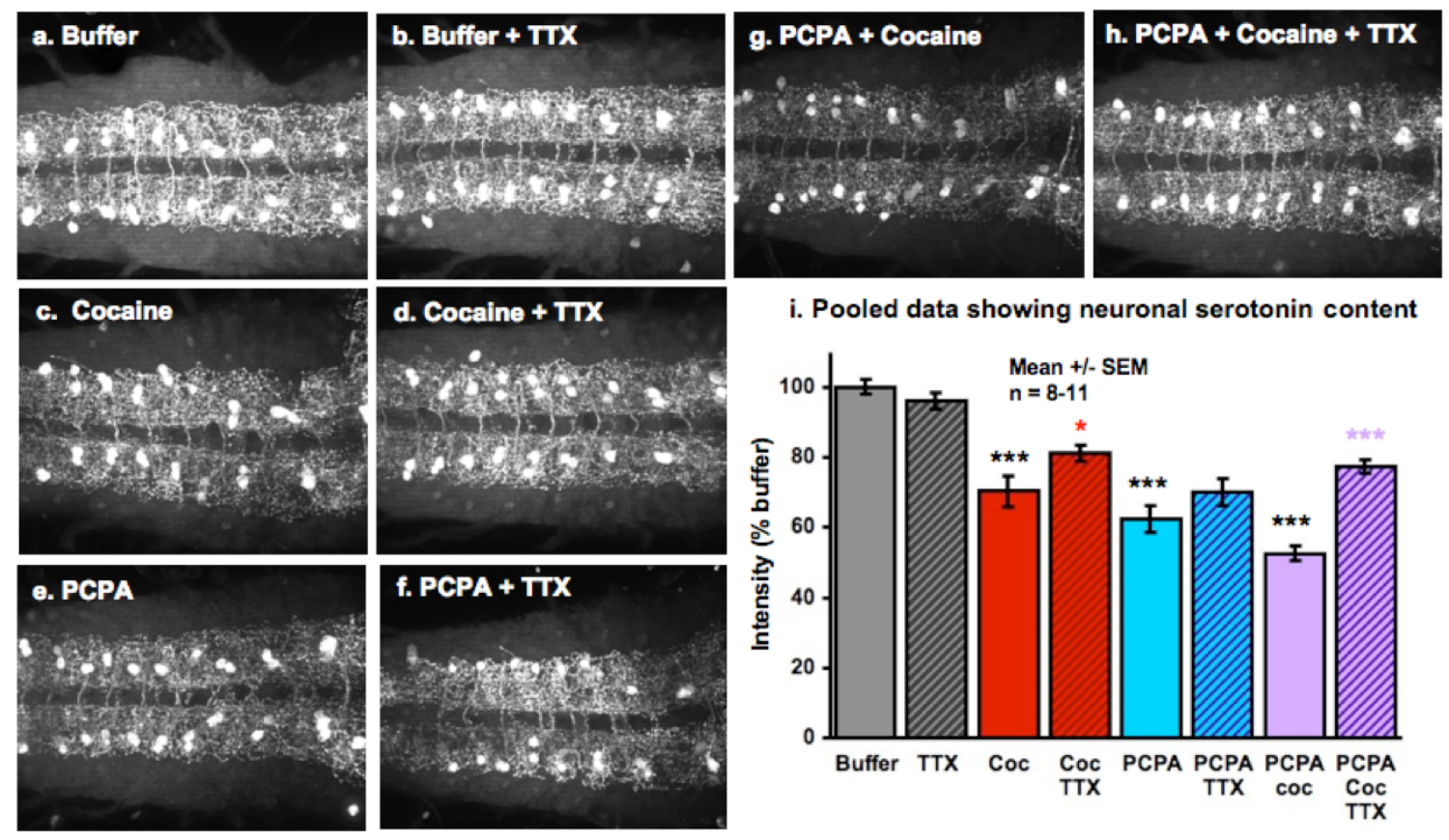

i. Pooled data showing neuronal serotonin content

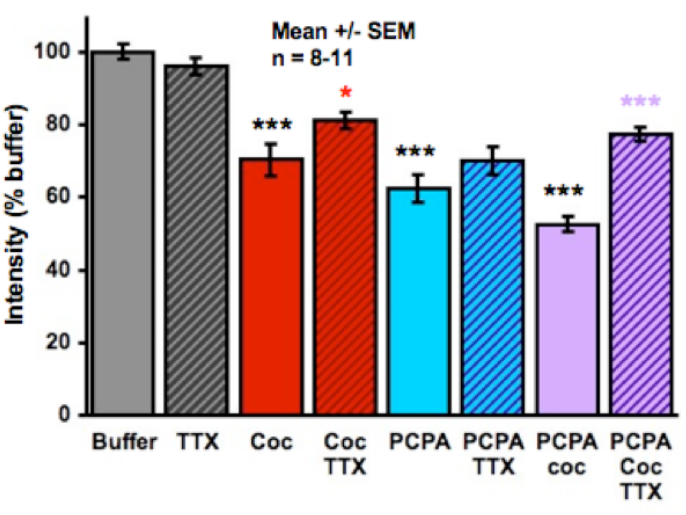

Figure 2. Effect of reuptake, synthesis, and activity inhibition on neuronal serotonin content. To quantify neuronal serotonin levels, we performed immunohistochemistry for the neurotransmitter. Representative images from VNCs stained for serotonin (white) after 30 minute incubation in buffer or drugs are shown in a-h. (a) VNC dissected in buffer shows normal serotonin content. (b) VNC incubated in tetrodotoxin (TTX) is similar to buffer. (c) VNC incubated in $10 \mu \mathrm{M}$ cocaine is dimmer than buffer, indicating lower serotonin content, but concurrent incubation with TTX $(d)$ is brighter than cocaine alone. This indicates that TTX ameliorates the serotonin depletion. (e) $100 \mu \mathrm{M} \mathrm{PCPA}$ is dimmer than buffer and incubation in PCPA + TTX (f) is brighter than PCPA alone. (g) $100 \mu \mathrm{M}$ PCPA plus $10 \mu \mathrm{M}$ cocaine appears dimmer than incubation in buffer or either drug alone. (h) Incubation in PCPA, cocaine and TTX is brighter, indicating serotonin levels are restored. (i) Pooled data show intensity of serotonin staining quantified as a $\%$ of staining intensity in concurrently performed buffer controls. Black asterisks in columns 3, 5, and 7 indicate a significant decrease in intensity from control VNCs incubated in buffer (One way ANOVA with Bonferroni's post-test, see Table 1 for values). Colored asterisks in columns 2, 4, 6, and 8 indicate a significant increase, over drug alone, in staining intensity in VNCs exposed to TTX (student t-test, 2 tailed). * $p$ $<0.05,{ }^{* *} p<0.01,{ }^{* * *} p<0.001$. Serotonin levels in VNCs incubated in both PCPA and cocaine are significantly lower than those incubated in either drug alone (One way ANOVA with Bonferroni's post-test, $p=0.02$ PCPA, $p=0.002$ cocaine). 
SSRIs are expected to decrease serotonin content because released serotonin cannot be recycled by the transporter. Reuptake was inhibited with $10 \mu \mathrm{M}$ cocaine. Although typically associated with dopamine transporter inhibition in mammals, cocaine has 6-fold greater affinity for SERT in the fly and offers greater selectivity for SERT than SSRIs like fluoxetine [12]. When reuptake is inhibited, neuronal serotonin content decreases, as shown in the representative image from a cocaine-incubated VNC that is dimmer than the control (Fig. 2c). Concurrent application of TTX with cocaine ameliorates the decreases in staining intensity (Fig. 2d). Pooled data show a significant reduction in serotonin content after cocaine incubation, to about $70 \%$ of buffer controls (One-way ANOVA with Bonferroni's multiple comparison post-test, $p<0.001$, see table 1). Serotonin content significantly increases when activity is concurrently inhibited by TTX compared to cocaine alone (student t-test, $\mathrm{p}=0.002)$ (Fig. 2i).

\begin{tabular}{|l|c|l|}
\hline \multicolumn{1}{|c|}{ Comparison } & $\mathbf{t}$ & \multicolumn{1}{c|}{ P value } \\
\hline Buffer vs Coc & 6.972 & $\mathrm{P}<0.001$ \\
\hline Buffer vs PCPA & 5.702 & $\mathrm{P}<0.001$ \\
\hline Buffer vs PCPA + Coc & 9.105 & $\mathrm{P}<0.001$ \\
\hline Coc vs PCPA & 1.627 & $\mathrm{P}>0.05$ \\
\hline Coc vs PCPA + Coc & 3.858 & $\mathrm{P}<0.01$ \\
\hline PCPA vs PCPA + Coc & 2.034 & $\mathrm{P}>0.05$ \\
\hline
\end{tabular}

Table 1. One way ANOVA of data shown in figure 2. One way ANOVA shows a significant (0.0001) difference in the serotonin content between groups. Table shows Bonferroni's comparison post tests. Significant values are highlighted. 
Synthesis should also be important for maintaining the neuronal serotonin pool and we tested this by applying p-chloroalanine (PCPA), which inhibits the serotonin synthesis enzyme Tryptophan hydroxylase2 (Tph2). Neuronal serotonin content decreases after a 30 minute incubation in $100 \mu \mathrm{M}$ PCPA (Fig. 2e). The decrease in staining intensity is especially prominent in the serotonergic projections, with the most proximal and distal abdominal segments being most affected. Pooled data show that levels are significantly smaller, about $60 \%$ of buffer controls (One-way ANOVA with Bonferroni's multiple comparison post-test, $\mathrm{p}<0.001$, see table 1). A significant increase in serotonin content is evident when activity is concurrently inhibited by TTX, compared to PCPA only (student t-test, $p=0.02)$ (Fig. 2f, i).

When both synthesis and reuptake are inhibited, neuronal serotonin content decreases significantly to $~ 50 \%$ of that in buffer (One-way ANOVA with Bonferroni's multiple comparison post-test, $p<0.001$, see table 1). Pooled data shows that MGLs from VNCs incubated in a combination of PCPA and cocaine are significantly smaller than just cocaine (One-way ANOVA with Bonferroni's multiple comparison post-test, $\mathrm{p}<$ 0.01). A non-significant trend of the combination being smaller than just PCPA was also observed (One-way ANOVA with Bonferroni's multiple comparison post-test, $p>0.05$, see table 1). MGL in these VNCs increase to $~ 80 \%$ of control when TTX is also present, a significant increase over the combination alone (Students t-test, $p<0.0001$ ).

We observed that synthesis or reuptake inhibition decreased serotonin content by about one-third, and that inhibition of endogenous activity by TTX rescued about a quarter of the decrease. The fact that TTX did not restore serotonin content to original levels means that either activity was not fully blocked or that some serotonin was lost through a different mechanism. Concurrent synthesis and reuptake inhibition decreased serotonin content by about half, a larger effect than separate drug administration. From these results, we formed simple predictions about the effect of pharmacological inhibition 
of reuptake, synthesis, and neuronal activity on the releasable pool of serotonin. We hypothesized that less serotonin would be available for release after synthesis or reuptake inhibition, with activity inhibition rescuing the amount of available serotonin. We also hypothesized that concurrent synthesis and uptake inhibition would deplete the releasable pool more than separate synthesis or uptake inhibition. To directly test these hypotheses and gain a more precise understanding of the effect of reuptake and synthesis inhibition on the serotonin pool, an analysis of endogenous serotonin release was carried out using direct electrochemical detection.

\section{ii. Characterization of stimulated serotonin release in the fly}

To elicit and detect serotonin release in larval Drosophila, we utilized a novel combination of methods as described previously [13]. Serotonin release was controlled optically, by Channelrhodopsin-2 (ChR2), a blue light-activated, cation-selective ion channel that was expressed under the control of the serotonergic neuron-specific driver, Tph-GAL4 [20]. To detect serotonin, we used fast-scan cyclic voltammetry (FSCV) at an implanted carbon-fiber microelectrode, which directly measured serotonin in the extracellular space in real time [21]. Electrodes were implanted into the neuropil of isolated Drosophila VNCs from wandering third instar larvae, which exhibit a mature serotonergic system [22].

Figure 3 shows raw data of serotonin release elicited by exposing the VNC to blue light for $10 \mathrm{~s}$. The peaks in the CV collected in a VNC expressing ChR2 dissected in buffer (Fig 3a, black line) are located at the same voltages as the $1 \mu \mathrm{M}$ serotonin electrode calibration CV (red line), confirming that serotonin was detected. Pharmacological experiments have also previously confirmed the identity of the neurotransmitter as serotonin [13]. Fig. 3b shows serotonin concentration changes over time for two consecutive stimulations, performed in the same sample 2 min apart. The 
peak height is decreased for the second stimulation but the time course is comparable. The current at the oxidation voltage for serotonin was converted to concentration based on electrode calibration data.
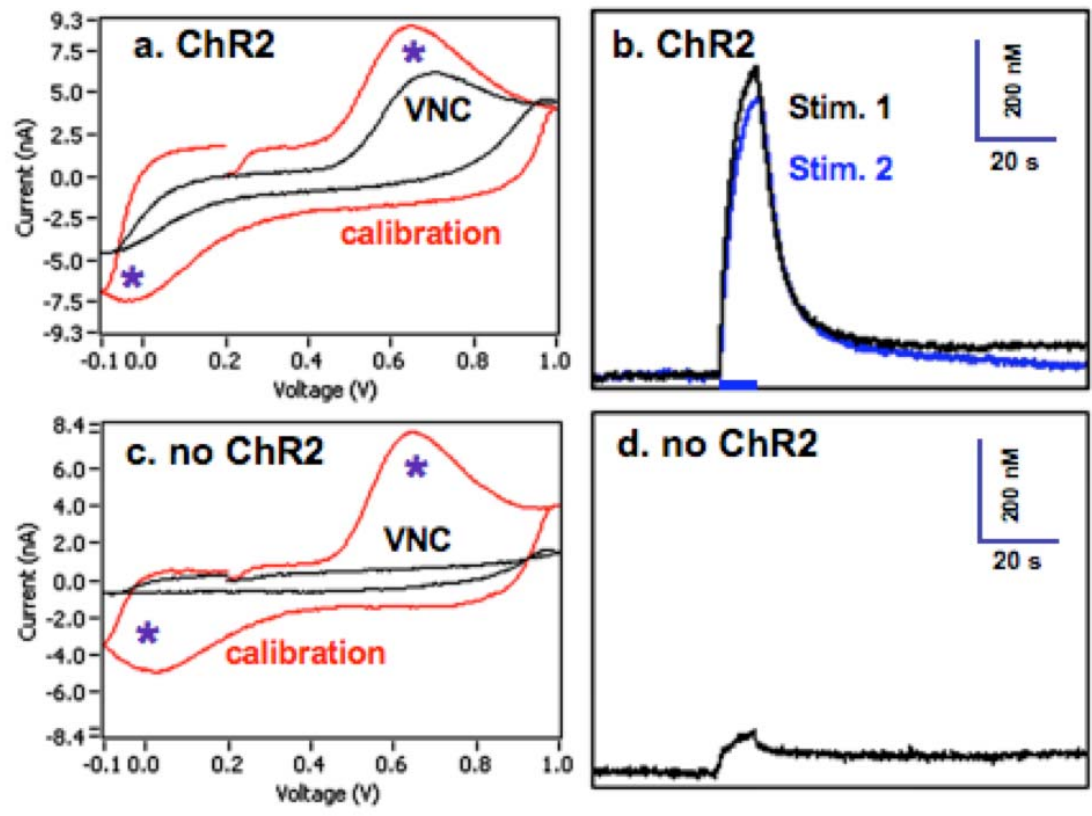

Figure 3. Raw data of evoked serotonin rlease. (a-b) VNC expressing ChR2 dissected in buffer. (a) The background-subtracted CV verifies that serotonin is being detected because the $\mathrm{CV}$ from the sample (black line) is similar to that obtained during electrode calibration with $1 \mu \mathrm{M}$ serotonin (red line). The asterisks identify the oxidation and reduction peaks. (b) Concentration vs time traces show neurotransmitter concentration changes over time during a 10 s stimulation (duration marked by blue line underneath trace). The peak from the second stimulation (blue) is smaller than the first (black) but comparable in time course. Stimulations were performed 2 min. apart. (c-d) Control VNC lacking ChR2 expression. (c) The sample CV (black line) does not show any neurotransmitter specific peaks (as in the calibration, red line). (d) Concentration vs trace shows a small fluctuation in current resulting from other ionic and electrical changes. This signal is an error, not serotonin, and is about $10 \%$ of normal release.

Serotonin release was not detectable in control VNCs not expressing ChR2 in serotonergic neurons. For example, a representative CV from a control VNC does not show any peaks characteristic of serotonin that appear in the CV from the electrode 
calibration (Fig. 3c). The concentration vs. time trace, at the potential for serotonin oxidation (Fig 3d) shows a small fluctuation due to noise, likely from blue light-induced ionic changes that affect the background current. The data were quantified using two parameters, peak height and time to half-maximal signal decay $\left(t_{50}\right)$. Peak height is the maximal detected concentration and $t_{50}$ is the time from the end of the stimulation until the signal has decayed to half the peak height. For VNCs expressing ChR2 dissected in buffer, the initial $10 \mathrm{~s}$ stimulation elicits a peak serotonin concentration of $600+/-30 \mathrm{nM}$ and $a t_{50}$ of $4.6+/-0.3 \mathrm{~s}(\mathrm{SEM}, \mathrm{n}=35)$.

\section{iii. Effects of multiple stimulations.}

To estimate the rate at which serotonergic neurons can replenish the releasable pool of serotonin, we performed multiple stimulations $1,2,5$, or 10 minutes apart. As shown in Figure $4 \mathrm{a}$ and $\mathrm{b}$, there was no significant difference in average peak height or $t_{50}$ for the initial stimulation between sample groups. Figure $3 c$ compares averaged data from multiple stimulations repeated at different intervals. Data were normalized to the initial peak height for each animal because peak concentrations can vary between VNCs (range of $~ 200 \mathrm{nM}$ ). When stimulations were performed either 1 or 2 minutes apart, a progressive decrease in peak height is evident. The extent of decay was not significantly different for stimulations performed 1 or 2 min apart (Two-way ANOVA with Bonferroni's multiple comparison post-test, $p>0.05)$. However, when stimulations are performed every 5 or 10 minutes, the signal remains relatively stable and the $8^{\text {th }}$ stimulation evokes peak concentrations similar to the first (Fig. 4c). Therefore, the replenishment of the releasable pool normally occurs on a time scale between 2 and $5 \mathrm{~min}$. Because the largest signal decay occurs within the first 4 stimulations, we chose to focus our analysis on these stimulations for most of the subsequent experiments involving pharmacological manipulations. 

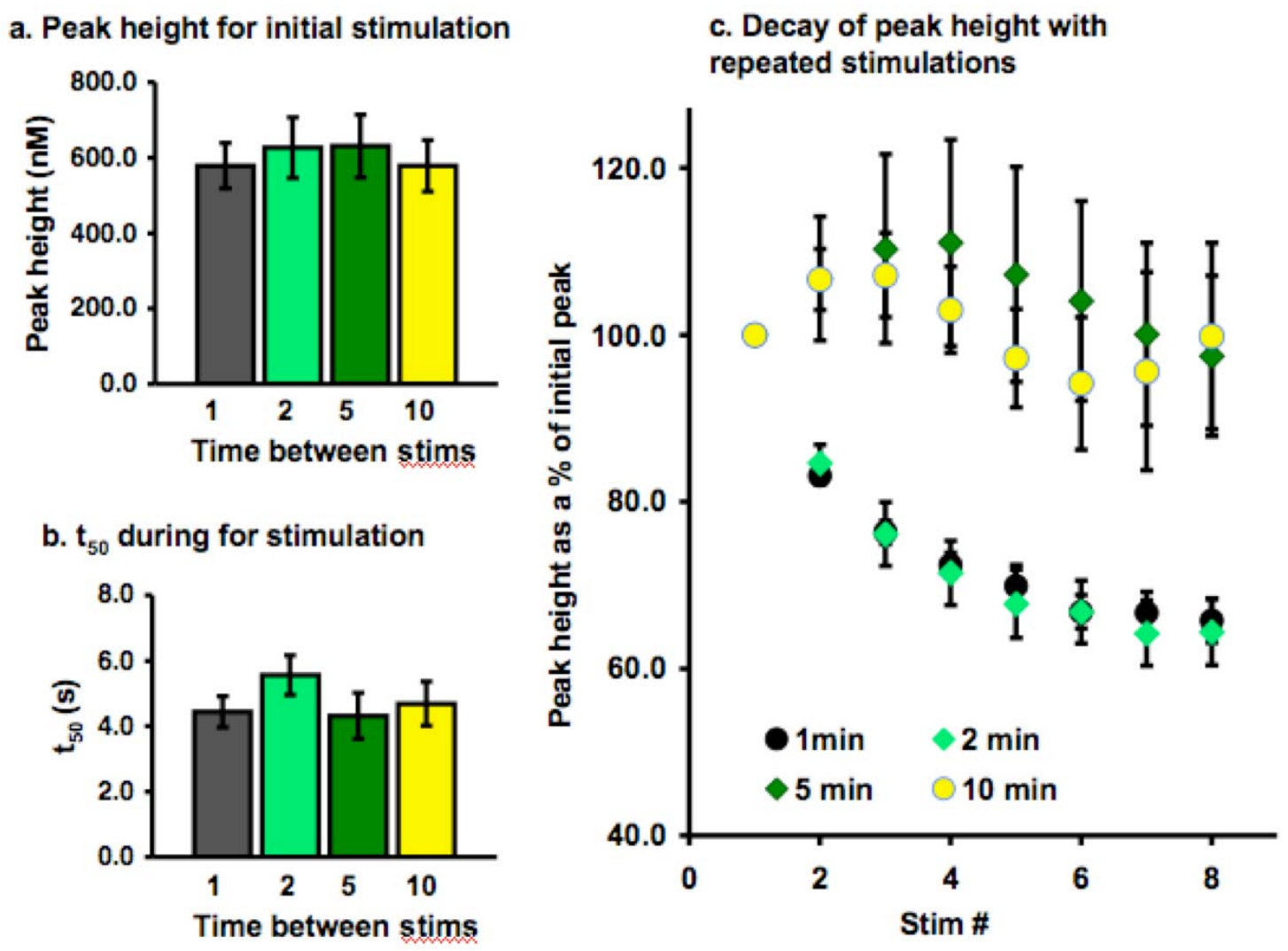

Figure 4. Serotonin release during stimulations repeated at 1, 2, 5 or 10 min intervals. Pooled data are mean +/- SEM, $n=6-9$. (a) The peak height for the initial stimulation is not significantly different for the different groups (Two-way ANOVA with Bonferonni posttest, $p>0.05$ ). (b) $t_{50}$, the time to half decay, is also not different for the different groups (Two-way ANOVA with Bonferonni post-test, p > 0.05). (c) A plot of normalized peak height vs stimulation number shows that peak height decays the most during the first 4 stimulations when they are performed 1 or 2 minutes apart. Signal is more stable when stimulations are performed 5 or 10 min apart.

\section{iv. Effect of reuptake inhibition on the releasable pool of serotonin}

Cocaine is known to inhibit uptake and our previous studies have shown that it increases the time course of serotonergic signaling (Borue et al, 2009). Indeed, in this study, incubation in $10 \mu \mathrm{M}$ cocaine did not have a significant effect on the initial peak height of stimulation, but did significantly increase $t_{50}$ (Fig. 5). The lack of effect on initial peak height is somewhat surprising, given the reduction in neuronal serotonin content 
observed by staining (Fig. 2c). However, uptake inhibition is expected to increase the peak height if uptake normally occurs simultaneously with release, since less serotonin would be cleared during the rising phase. Therefore, the combination of these effects may lead to the trend towards a non-significant decrease in evoked serotonin. An alternative explanation is that not all of the neuronal serotonin may be available for release.

Peak height comparisons

\begin{tabular}{|l|c|c|}
\hline \multicolumn{1}{|c|}{ Comparison } & t value & P value \\
\hline Buffer vs TTX & 3.944 & $P<0.01$ \\
\hline Buffer vs Coc & 1.825 & $P>0.05$ \\
\hline Buffer vs PCPA & 2.269 & $P>0.05$ \\
\hline Buffer vs PCPA + Coc & 7.61 & $P<0.001$ \\
\hline Buffer vs PCPA + Coc +TTX & 0.304 & $P>0.05$ \\
\hline Coc vs PCPA & 0.508 & $P>0.05$ \\
\hline Coc vs PCPA + Coc & 5.451 & $P<0.001$ \\
\hline PCPA vs PCPA + Coc & 4.766 & $P<0.001$ \\
\hline TTX vs PCPA + Coc + TTX & 2.616 & $P>0.05$ \\
\hline
\end{tabular}

$t_{50}$ comparisons

\begin{tabular}{|l|c|c|}
\hline \multicolumn{1}{|c|}{ Comparison } & t value & P value \\
\hline Buffer vs TTX & 0.838 & $P>0.05$ \\
\hline Buffer vs Coc & 12.72 & $P<0.001$ \\
\hline Buffer vs PCPA & 0.324 & $P>0.05$ \\
\hline Buffer vs PCPA + Coc & 13.42 & $P<0.001$ \\
\hline Buffer vs PCPA + Coc +TTX & 8.448 & $P<0.001$ \\
\hline Coc vs PCPA & 9.983 & $P<0.001$ \\
\hline Coc vs PCPA + Coc & 3.674 & $P<0.01$ \\
\hline PCPA vs PCPA + Coc & 11.57 & $P<0.001$ \\
\hline TIX vs PCPA + Coc + TTX & 6.392 & $P<0.001$ \\
\hline
\end{tabular}

Table 2. One way ANOVA of data shown in figure 5 (next page). One way ANOVA shows a significant $(0.0001)$ difference in peak height or t50. Table shows Bonferroni's comparison post tests. Significant values are highlighted. 

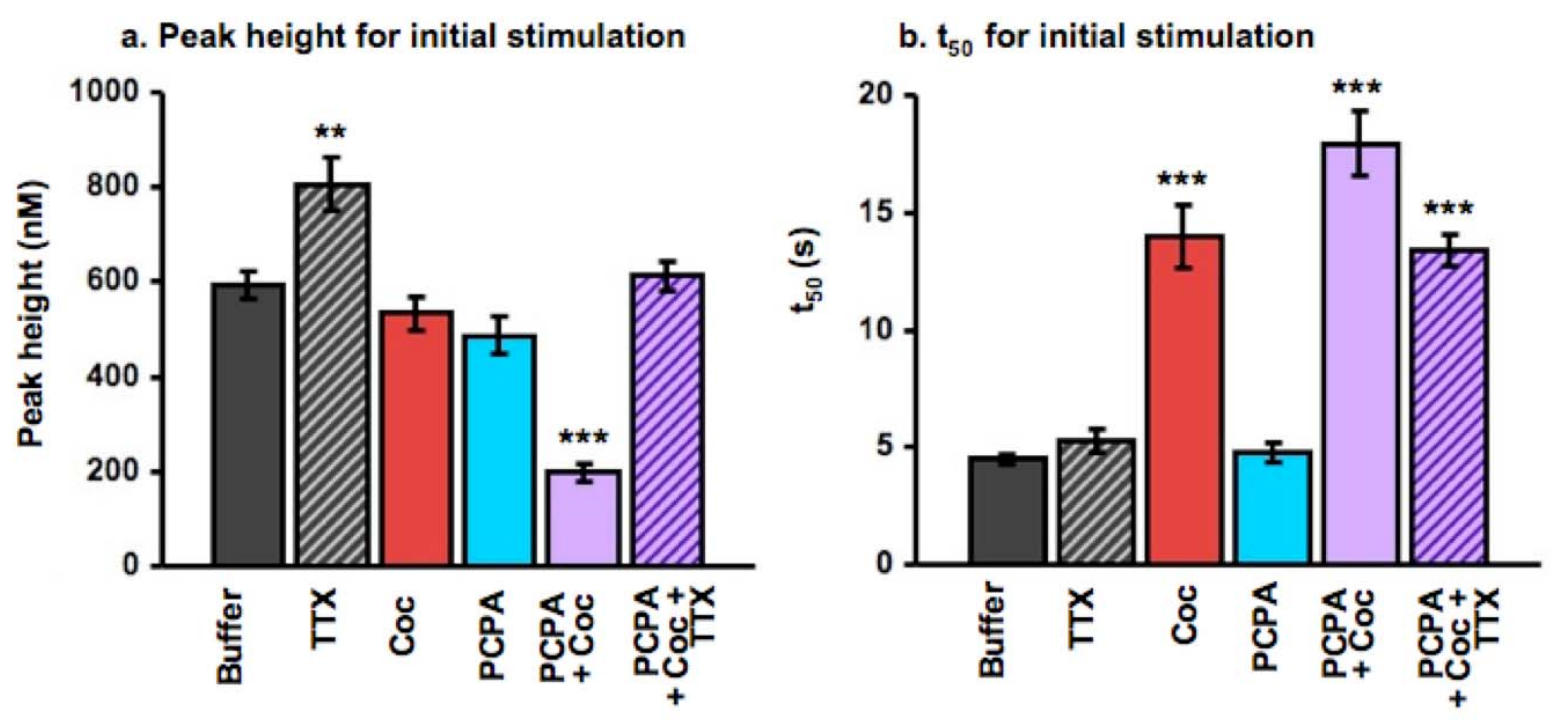

Figure 5. Effect of pharmacological agents on serotonin release and clearance on initial stimulation. To test the effect of inhibiting synthesis, reuptake, or neuronal activity on stimulated serotonin release, VNCs were dissected and incubated for $30 \mathrm{~min}$ in the presence of drugs. Data are mean +/- SEM ( $n=12-26)$. (a) Peak height for initial $10 \mathrm{~s}$ stimulation. A trend toward smaller peak height is observed in VNCs incubated in $10 \mu \mathrm{M}$ cocaine and in $100 \mu \mathrm{M}$ PCPA. Incubation in both $100 \mu \mathrm{M}$ PCPA and $10 \mu \mathrm{M}$ cocaine significantly decreases peak height (One-way ANOVA with Bonferonni post-test, See Table 2 for $p$ values for the charts in this figure). Significant deviation from values in buffer are denoted by printed asterisks ( ${ }^{*} p<0.05$; $\left.{ }^{* *} p<0.01 ;{ }^{* * *} p<0.001\right)$. Incubation in tetrodotoxin (TTX) alone leads to significantly larger peak height as compared to buffer. In combination with PCPA and cocaine, peak height is restored to control levels after TTX. (b) $t_{50}$ is significantly increased, indicating slower serotonin clearance, in VNCs exposed to cocaine but not PCPA or TTX alone (One-way ANOVA with Bonferonni post-test).

To test the effects of cocaine on depletion of evoked serotonin, we performed multiple stimulation experiments on VNCs incubated in the drug. The buffer experiments showed that the decay in peak height was not significantly different for stimulations performed every 1 or 2 minutes and likewise for stimulations performed every 5 or 10 minutes. Therefore, in Figure 6, we compare data from the 1 and 10 minute groups. When stimulations are performed every minute in the presence of cocaine, the peak 
height decays significantly more than the buffer group (Two-way ANOVA with Bonferroni's multiple comparison post-test, see table 3). On the fourth stimulation, the peak height is $76+/-3 \%$ of the initial value for VNCs dissected in buffer while it is only $54+/-3 \%$ for VNCs dissected in cocaine. Therefore, the peak concentration in cocainedissected VNCs decreases by about one-half for closely repeated stimulations.

a. Stimulations repeated $1 \mathrm{~min}$ apart

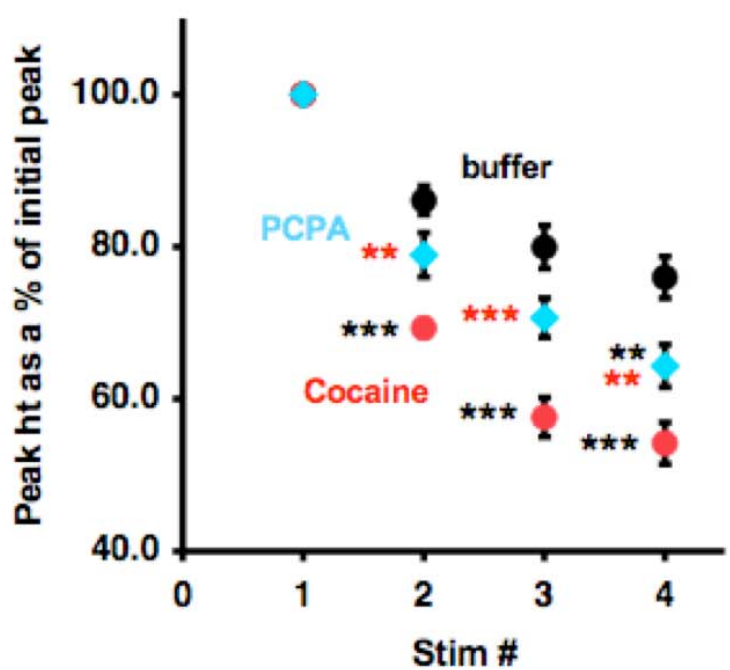

b. Stimulations repeated $10 \mathrm{~min}$ apart

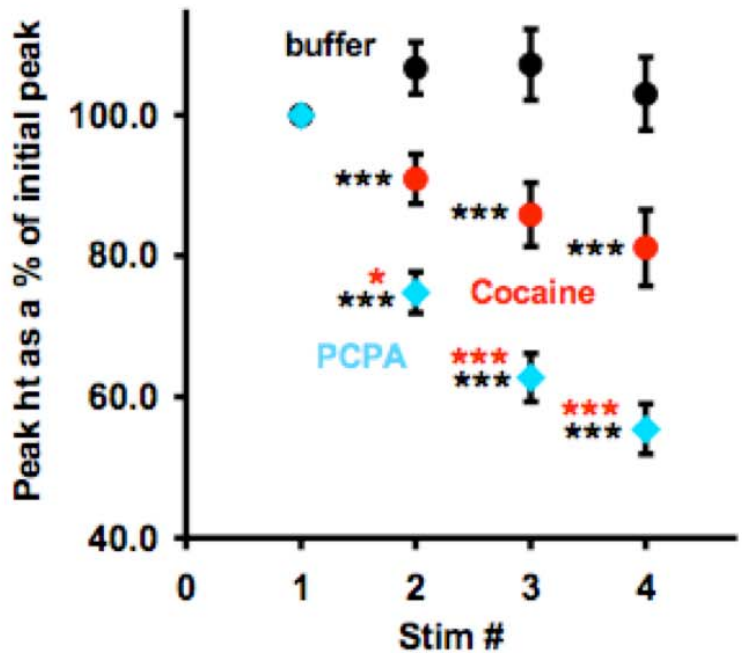

Figure 6. Effect of reuptake and synthesis inhibition on repeated stimulations. Serotonin release during repeated stimulations is affected by a serotonin transport inhibitor, $10 \mu \mathrm{M}$ cocaine (red) or a serotonin synthesis inhibitor, $100 \mu \mathrm{M}$ PCPA (blue). Pooled data (mean +/- SEM) show the normalized peak height across 4 stimulations performed 1 minute, $n=6-9$ (a) or 10 minutes, $n=4-6$ (b) apart. Significant deviation (Two-way ANOVA with Bonferroni's post-test, see table 3) from peak height of samples dissected in buffer (black) is indicated by black asterisks and differences between PCPA and cocaine are indicated by red asterisks next to PCPA values $\left({ }^{*} p<0.05\right.$; ${ }^{* *} p<0.01$; ${ }^{* * *} p<0.001$ ). Note the difference in trends for 1 vs 10 minute stimulations (see Fig. 2 for overlaid graphs). 
Stims 1 min apart

\begin{tabular}{|l|c|c|}
\hline \multicolumn{3}{|c|}{ Buffer vs Cocaine } \\
\hline Stim number & $\mathbf{t}$ & P value \\
\hline Stim 1 & 0 & $\mathrm{P}>0.05$ \\
\hline Stim 2 & 4.88 & $\mathrm{P}<0.001$ \\
\hline Stim 3 & 6.81 & $\mathrm{P}<0.001$ \\
\hline Stim 4 & 6.52 & $\mathrm{P}<0.001$ \\
\hline
\end{tabular}

\begin{tabular}{|l|c|c|}
\hline \multicolumn{3}{|c|}{ Buffer vs PCPA } \\
\hline Stim number & $\mathbf{t}$ & $\mathbf{P}$ value \\
\hline Stim 1 & 0 & $\mathrm{P}>0.05$ \\
\hline Stim 2 & 1.66 & $\mathrm{P}>0.05$ \\
\hline Stim 3 & 2.11 & $\mathrm{P}<0.05$ \\
\hline Stim 4 & 3.18 & $\mathrm{P}<0.01$ \\
\hline
\end{tabular}

\begin{tabular}{|l|c|c|}
\hline \multicolumn{3}{|c|}{ Cocaine vs PCPA } \\
\hline Stim number & $\mathbf{t}$ & P value \\
\hline Stim 1 & 0 & $\mathrm{P}>0.05$ \\
\hline Stim 2 & 3.27 & $\mathrm{P}<0.01$ \\
\hline Stim 3 & 4.66 & $\mathrm{P}<0.001$ \\
\hline Stim 4 & 3.51 & $\mathrm{P}<0.01$ \\
\hline
\end{tabular}

Stims 10 min apart

\begin{tabular}{|l|c|c|}
\hline \multicolumn{3}{|c|}{ Buffer vs Cocaine } \\
\hline Stim number & $\mathbf{t}$ & P value \\
\hline Stim 1 & 0 & $\mathrm{P}>0.05$ \\
\hline Stim 2 & 2.8 & $\mathrm{P}<0.05$ \\
\hline Stim 3 & 3.8 & $\mathrm{P}<0.01$ \\
\hline Stim 4 & 3.89 & $\mathrm{P}<0.01$ \\
\hline
\end{tabular}

\begin{tabular}{|l|c|c|}
\hline \multicolumn{3}{|c|}{ Buffer vs PCPA } \\
\hline Stim number & $\mathbf{t}$ & P value \\
\hline Stim 1 & 0 & $\mathrm{P}>0.05$ \\
\hline Stim 2 & 6.04 & $\mathrm{P}<0.001$ \\
\hline Stim 3 & 8.44 & $\mathrm{P}<0.001$ \\
\hline Stim 4 & 9.03 & $\mathrm{P}<0.001$ \\
\hline
\end{tabular}

\begin{tabular}{|l|c|c|}
\hline \multicolumn{3}{|c|}{ Cocaine vs PCPA } \\
\hline Stim number & $\mathbf{t}$ & P value \\
\hline Stim 1 & 0 & $\mathrm{P}>0.05$ \\
\hline Stim 2 & 2.76 & $\mathrm{P}<0.05$ \\
\hline Stim 3 & 3.96 & $\mathrm{P}<0.001$ \\
\hline Stim 4 & 4.41 & $\mathrm{P}<0.001$ \\
\hline
\end{tabular}

Table 3. Two way ANOVA of data shown in figure 6. ANOVA shows a significant $(0.0001)$ interaction of time and group. Table shows Bonferroni's comparison post tests . Significant values are highlighted.

VNCs stimulated every 10 minutes in the presence of cocaine show significantly smaller peak heights for stimulations 2 to 4 (Fig. 6b). In contrast, when stimulations are performed every 10 minutes in buffer, the peak height remains stable. For stimulations repeated at longer intervals, the decay by the fourth stimulation is only about one-fifth of the initial stimulation. This decay in peak height with 10 min stimulations is significantly less than that observed when stimulations were performed 1 min apart (Fig. 7, Two-way 
ANOVA with Bonferroni's multiple comparison post-test, see table 4). Therefore, the reuptake inhibition has a greater effect for closely repeated stimulations.

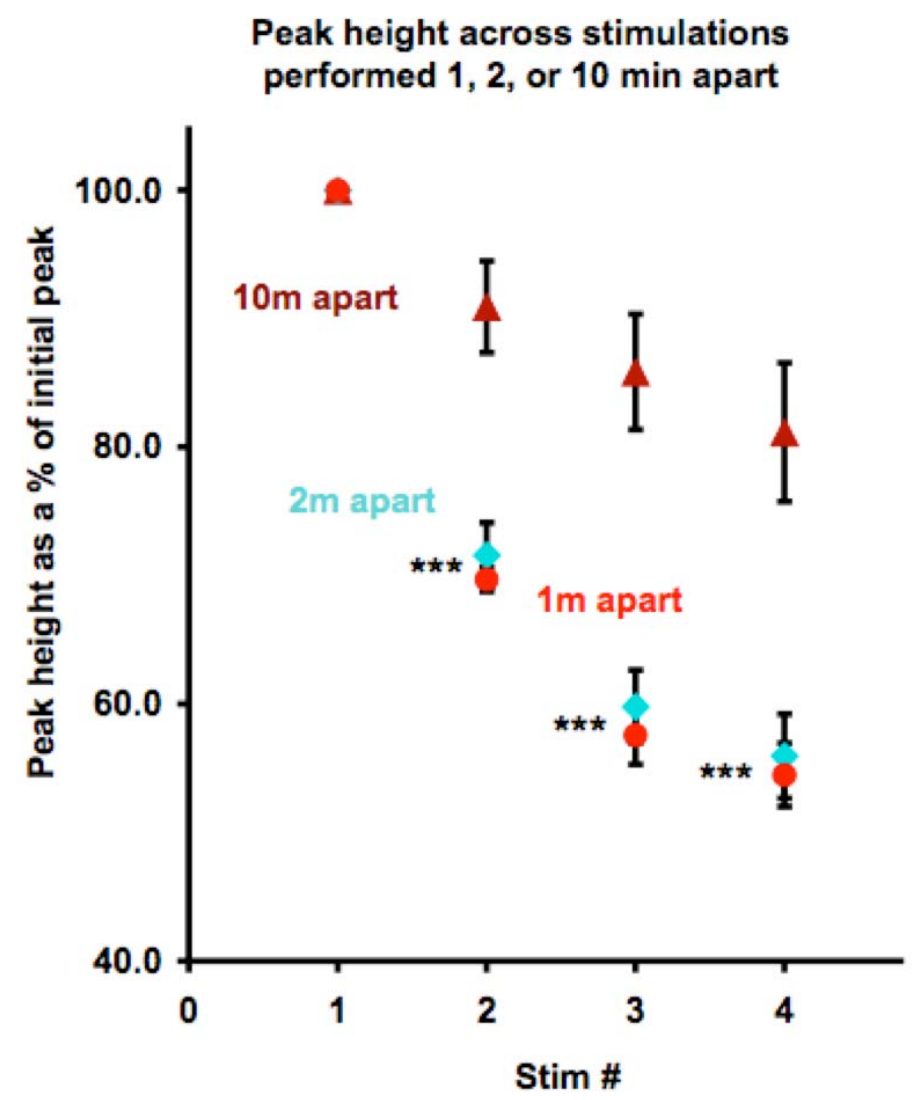

Figure 7. Serotonin depletion in the presence of cocaine depends on inter-stimulation time. Pooled data of peak height normalized as a \% of initial peak height across stimulations performed 1 (red), 2 (blue), or 10 minutes apart (burgundy). This is an additional plot provided to allow a visual comparison of data from VNCs incubated in cocaine to show that more depletion occurs when stimulations are performed close together. Data is mean +/- SEM, $n=4-6$. Peaks are significantly different in height when stimuli are performed 10 as opposed to 1 or 2 minutes apart (Two way ANOVA, $p<$ 0.001 , see table 4). 


\begin{tabular}{|l|c|c|}
\hline \multicolumn{3}{|c|}{ Cocaine 1 min vs 10 min } \\
\hline Stim number & $\mathbf{t}$ & P value \\
\hline Stim 1 & 0 & $\mathrm{P}>0.05$ \\
\hline Stim 2 & 5.58 & $\mathrm{P}<0.001$ \\
\hline Stim 3 & 7.43 & $\mathrm{P}<0.001$ \\
\hline Stim 4 & 7.01 & $\mathrm{P}<0.001$ \\
\hline
\end{tabular}

\begin{tabular}{|l|c|c|}
\hline \multicolumn{3}{|c|}{ PCPA 1 min vs 10 min } \\
\hline Stim number & $\mathbf{t}$ & $\mathbf{P}$ value \\
\hline Stim 1 & 0 & $\mathrm{P}>0.05$ \\
\hline Stim 2 & 0.98 & $\mathrm{P}>0.05$ \\
\hline Stim 3 & 1.90 & $\mathrm{P}>0.05$ \\
\hline Stim 4 & 2.14 & $\mathrm{P}>0.05$ \\
\hline
\end{tabular}

Table 4. Two way ANOVA of data shown in figures 6,7. Showing the effect of interstimulation time on stimulated release in the presence of cocaine or PCPA. ANOVA shows a significant $(0.0001)$ interaction of time and group for cocaine and time only for PCPA. Table shows Bonferroni's comparison post tests. Significant values are highlighted.

\section{v. Effect of synthesis inhibition on stimulated release.}

Blocking synthesis with PCPA should also reduce the amount of serotonin available for release. Fig. 5 shows that incubation in $100 \mu \mathrm{M}$ PCPA shows a trend towards slightly smaller release on the first stimulation and no change in $t_{50}$ (One way ANOVA with Bonferonni post-test, $p>0.05$ ). Similar to the cocaine results, although tissue content is reduced by about one-third after PCPA (Fig. 2 e), the initial stimulation is not significantly reduced. This again suggests that not all of the serotonin measured in buffer controls may be available for release.

When stimulations are performed every minute in the presence of PCPA, a trend is evident towards smaller release than buffer controls on stimulations 2 and 3 (Fig. 6a). This trend becomes significant by the $4^{\text {th }}$ stimulation when the peak height is $64+/-3 \%$ of the initial value (Two-way ANOVA with Bonferroni's multiple comparison post-test, see table 3). When stimulations are performed every $10 \mathrm{~min}$ in the presence of PCPA, a significantly smaller peak height is also evident for stimulations 2 to 4 (Fig. 6b). The decay in peak height is very similar for the stimulations performed every 1 or 10 minutes with PCPA (data not significantly different, two-way ANOVA with Bonferroni's multiple 
comparison post-test, see table 4). The inter-stimulation time therefore does not play as big of a role when synthesis is inhibited and peak height after four stimulations is about one third of the initial height, regardless of the time between stimulations.

Comparing the PCPA and cocaine data, VNCs dissected in cocaine have a faster decay in signal compared to those dissected in PCPA with closely repeated stimulations (Fig. 6a). The normalized peak heights for the second, third and fourth stimulation are significantly lower in cocaine than in PCPA (Two-way ANOVA with Bonferroni's multiple comparison post-test, see table 3). For example, the second stimulation is $10 \%$ lower in cocaine than in PCPA. The trends for decay in peak heights are different for PCPA and cocaine when stimulations are repeated $10 \mathrm{~min}$. apart. For these stimulations, PCPA values are significantly lower than cocaine values for stimulations 2 to 4 (Two-way ANOVA with Bonferroni's multiple comparison post-test, see table 3). Therefore, cocaine and PCPA have different effect based on the interval of stimulation repetition. During closely repeated stimulations, VNCs show a faster decrease in peak height after cocaine while PCPA causes a greater decrease when stimulations are performed 10 minutes apart.

\section{vi. Effects of combined synthesis and reuptake inhibition on stimulated release}

The experiments discussed in the previous section strongly suggested that both synthesis and reuptake play key roles in the replenishment of the releasable pool of serotonin. To determine if these observed effects are additive, we pharmacologically inhibited both mechanisms. VNCs were dissected in a combination of $100 \mu \mathrm{M}$ PCPA and $10 \mu \mathrm{M}$ cocaine and allowed to incubate for 30 minutes. Raw data from these VNCs (Fig. 8) show a severely diminished peak concentration, even on the first stimulation; however the CV shows small peaks at the serotonin oxidation and reduction potentials, indicating 
serotonin is released. The time course of signaling $\left(t_{50}\right)$ is also prolonged, typical of serotonin signaling in the presence of a reuptake inhibitor.

VNCs exposed to both drugs exhibited a significant reduction of the initial peak height to about one-third of the buffer value (Fig. 5a). This is also significantly lower than the initial peak heights for VNCs dissected in PCPA or cocaine alone (One-way ANOVA with Bonferroni's multiple comparison post-test, table 2). The $t_{50}$ in VNCs exposed to both drugs is significantly greater than those dissected in buffer and slightly higher than observed with cocaine alone (One-way ANOVA with Bonferroni's multiple comparison post-test, table 2) (Fig. 5b). The latter effect is likely an artifact of the smaller peak height.
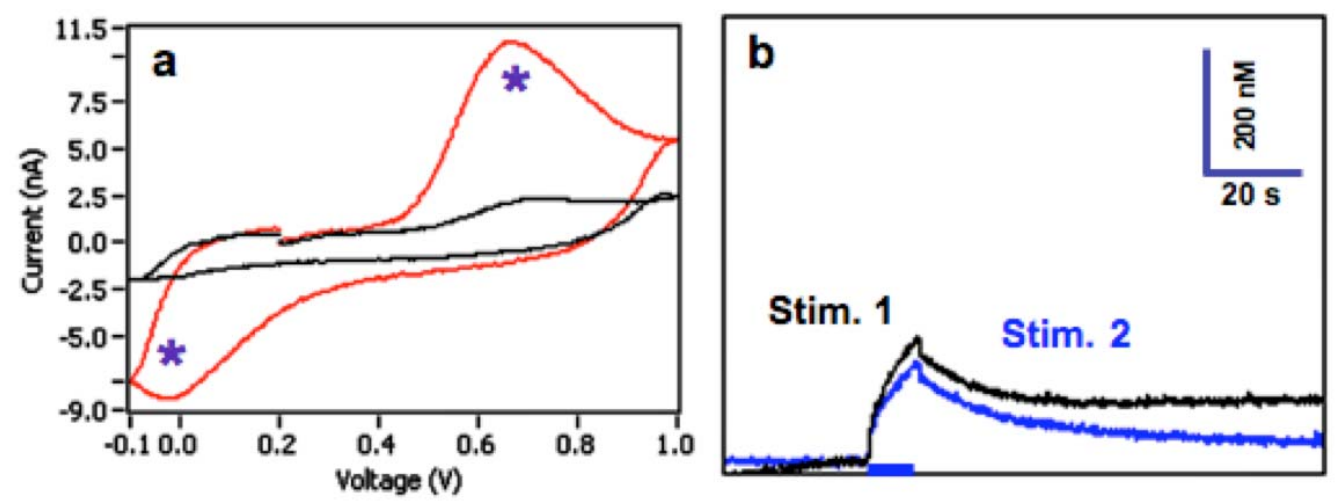

Figure 8. Raw data showing effect of cocaine and PCPA on serotonin release in Drosophila. Raw data from consecutive stimulations performed 2 minutes apart in the presence of both $10 \mu \mathrm{M}$ cocaine and $100 \mu \mathrm{M}$ PCPA (a). The CV confirms that a small amount of serotonin is released in these samples. (b) The peak height of these stimulations is much smaller than in buffer.

Comparing the neuronal content results to the stimulated release data suggests the presence of a threshold, below which decreased neuronal serotonin is evident during stimulated release. Synthesis or transport inhibition decrease neuronal content to $70 \%$ or $60 \%$ of buffer, respectively, without significantly decreasing initial peak height, while combined synthesis and reuptake inhibition decreases it to $50 \%$ of buffer with a 
concurrent decrease in initial peak height. Therefore, a large decrease in content may be necessary to cause changes in stimulated release.

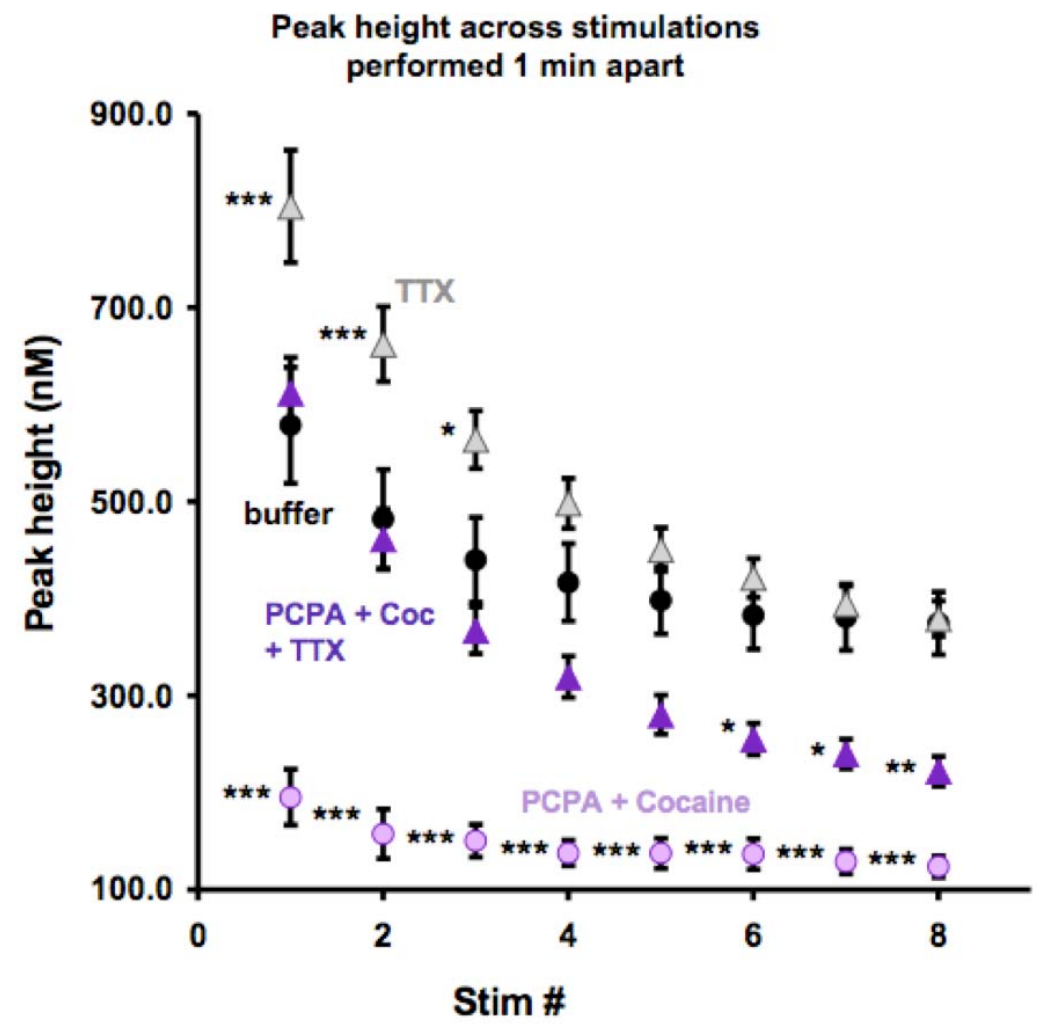

Figure 9. Neuronal activity affects evoked serotonin release. Pooled data, mean +/SEM, $\mathrm{n}=8-10$ showing peak height across multiple stimulations performed 1 min. apart. Because of the large difference in initial peak height from buffer, signals were not normalized. Smaller peaks occur during all stimulations when samples were incubated with $100 \mu \mathrm{M}$ PCPA + $10 \mu \mathrm{M}$ cocaine (light purple). In VNCs incubated in TTX (grey), peaks are initially larger, then decay to match those of buffer controls. When TTX is present in addition to cocaine and PCPA (dark purple), peak height is similar to buffer controls during the first few stimulations but then becomes significantly smaller. Significant deviation from values in buffer (black) are denoted by asterisks ( ${ }^{*} p<0.05$; ** $\left.p<0.01 ;{ }^{* * *} p<0.001\right)$.

Figure 9 shows the effects of concurrent PCPA and cocaine on the peak height of stimulations performed $1 \mathrm{~min}$ apart. Because of the large difference between initial peak height in buffer and the drug combination, we did not normalize the data. By 
stimulation 6 , the peak size decays below our detection limit to a level that is not significantly different from the noise signal observed in control VNCs that do not express ChR2 in serotonergic neurons (student t-test, $n=6-9, p=0.056)$. The CV from stimulations 6-8 does not show any serotonin-specific peaks, indicating that serotonin release was not observed. Therefore, when synthesis and reuptake are concurrently inhibited, 5 stimulations deplete the releasable pool of serotonin to below the limits of detection.

\begin{tabular}{|l|c|c|}
\hline \multicolumn{3}{|c|}{ Buffer vs TTX } \\
\hline Stim number & $\mathbf{t}$ & $\mathbf{P}$ value \\
\hline Stim 1 & 5.5 & $\mathrm{P}<0.001$ \\
\hline Stim 2 & 4.4 & $\mathrm{P}<0.001$ \\
\hline Stim 3 & 3 & $\mathrm{P}<0.05$ \\
\hline Stim 4 & 2 & $\mathrm{P}>0.05$ \\
\hline Stim 5 & 1.3 & $\mathrm{P}>0.05$ \\
\hline Stim 6 & 0.93 & $\mathrm{P}>0.05$ \\
\hline Stim 7 & 0.33 & $\mathrm{P}>0.05$ \\
\hline Stim 8 & 0.12 & $\mathrm{P}>0.05$ \\
\hline
\end{tabular}

\begin{tabular}{|l|c|c|}
\hline \multicolumn{3}{|c|}{ Buffer vs PCPA + Coc } \\
\hline Stim number & $\mathbf{t}$ & P value \\
\hline Stim 1 & 9.1 & $\mathrm{P}<0.001$ \\
\hline Stim 2 & 7.7 & $\mathrm{P}<0.001$ \\
\hline Stim 3 & 6.9 & $\mathrm{P}<0.001$ \\
\hline Stim 4 & 6.6 & $\mathrm{P}<0.001$ \\
\hline Stim 5 & 6.2 & $\mathrm{P}<0.001$ \\
\hline Stim 6 & 5.9 & $\mathrm{P}<0.001$ \\
\hline Stim 7 & 6 & $\mathrm{P}<0.001$ \\
\hline Stim 8 & 6 & $\mathrm{P}<0.001$ \\
\hline
\end{tabular}

\begin{tabular}{|l|c|c|}
\hline \multicolumn{3}{|c|}{ Buffer vs PCPA + Cocaine + TTX } \\
\hline Stim number & $\mathbf{t}$ & $\mathbf{P}$ value \\
\hline Stim 1 & 0.07 & $\mathrm{P}>0.05$ \\
\hline Stim 2 & 0.48 & $\mathrm{P}>0.05$ \\
\hline Stim 3 & 1.7 & $\mathrm{P}>0.05$ \\
\hline Stim 4 & 2.3 & $\mathrm{P}>0.05$ \\
\hline Stim 5 & 2.7 & $\mathrm{P}>0.05$ \\
\hline Stim 6 & 3 & $\mathrm{P}<0.05$ \\
\hline Stim 7 & 3.3 & $\mathrm{P}<0.05$ \\
\hline Stim 8 & 3.5 & $\mathrm{P}<0.01$ \\
\hline
\end{tabular}

\begin{tabular}{|l|c|c|}
\hline \multicolumn{3}{|c|}{ PCPA + Coc vS PCPA + Coc +TTX } \\
\hline Stim number & $\mathbf{t}$ & $\mathbf{P}$ value \\
\hline Stim 1 & 9.6 & $\mathrm{P}<0.001$ \\
\hline Stim 2 & 7 & $\mathrm{P}<0.001$ \\
\hline Stim 3 & 5 & $\mathrm{P}<0.001$ \\
\hline Stim 4 & 4.2 & $\mathrm{P}<0.001$ \\
\hline Stim 5 & 3.3 & $\mathrm{P}<0.01$ \\
\hline Stim 6 & 2.7 & $\mathrm{P}>0.05$ \\
\hline Stim 7 & 2.6 & $\mathrm{P}>0.05$ \\
\hline Stim 8 & 2.3 & $\mathrm{P}>0.05$ \\
\hline
\end{tabular}

Table 5. Two way ANOVA of data shown in figure 9. ANOVA shows a significant (0.0001) interaction of time and group. Table shows Bonferroni's comparison post tests. Significant values are highlighted. 


\section{vii. Effect of spontaneous activity on serotonin depletion}

To determine the extent to which spontaneous activity plays a role in the depletion of the releasable pool, we inhibited the propagation of action potentials with tetrodotoxin. We predicted that in serotonergic neurons, ChR2-mediated release would occur as a result of direct depolarization of the neurons, and would therefore continue to occur even when action potential propagation was inhibited. On the initial stimulation, VNCs incubated in $0.5 \mu \mathrm{M}$ TTX release significantly more serotonin, $800+/-60 \mathrm{nM}$, than those dissected in buffer (Fig. 5a). TTX did not have a significant effect on the $t_{50}$ (Oneway ANOVA with Bonferroni's multiple comparison post-test, table 2). This confirms that action potential propagation is not necessary for ChR2-mediated serotonin release and that inhibiting neuronal activity does not influence serotonin clearance. The data also show that endogenous activity depletes approximately one quarter of the releasable serotonin pool. These data contrast with the staining data where no increases in tissue content were observed after TTX inhibition.

To test whether the depletion of serotonin with PCPA and cocaine was due to spontaneous activity, action potential propagation was inhibited with TTX in the presence of PCPA and cocaine. The initial peak height with PCPA, cocaine and TTX is $610+/-30 \mathrm{nM}$ (Fig. 5a), significantly higher than when activity was not inhibited by TTX (One-way ANOVA with Bonferroni's multiple comparison post-test, table 2). The peak height with the 3 drug combination was not significantly different from that of VNCs dissected in buffer (One-way ANOVA with Bonferroni's multiple comparison post-test, table 2). The effect of TTX in conjunction with PCPA and cocaine, increasing the concentration $400 \mathrm{nM}$, is much larger than the effect of TTX on VNCs that were incubated in only buffer, where the increase was only $200 \mathrm{nM}$. Serotonin clearance rate is consistent with the presence of cocaine (Fig. 5b). 
While the initial stimulated concentration is the same for VNCs dissected in buffer and those dissected in the combination of TTX, PCPA, and cocaine, the pattern of release when stimulations for repeated stimulations was different (Fig. 9). When the drugs were given in combination, the peak height decreased more rapidly than the buffer control samples when stimulations were repeated every minute (peaks 6-8 are significantly lower than buffer for TTX, PCPA, and cocaine, Two-way ANOVA with Bonferroni's multiple comparison post-test, see table 5). This demonstrates that while TTX rescues the release for the initial stimulation, less serotonin is available for repeated stimulations.

\section{Discussion}

Although SSRIs are the current standard of care for depression, SSRI treatment is not always successful. SSRls presumably act by raising serotonin levels so understanding factors that control serotonin levels would give insight into the mechanism of action of SSRI treatments [23]. In this paper, we use measurements of stimulated serotonin release in Drosophila to show that serotonin synthesis, as well as reuptake, determines the size of the releasable pool. The effects of synthesis and reuptake inhibition are additive and substantial decreases are observed in neuronal serotonin content and evoked release when both are pharmacologically inhibited. Therefore, the effects of SSRIs may be influenced by synthesis.

\section{i. The releasable serotonin pool in Drosophila is influenced by neuronal activity}

To deplete the releasable pool, we applied large stimulations specifically to serotonergic neurons. Our stimulations likely represent a large response to a stressful situation. The initial stimulation released a large amount of serotonin, $\sim 600 \mathrm{nM}$, similar to electrical stimulations in the rat substantia nigra [21]. The decay in peak height for 
closely repeated stimuli implies that a significant portion of the pool was mobilized during our stimulations. When stimulations were performed 5 or more minutes apart, evoked release was stable, suggesting that the releasable pool is replenished in 2-5 minutes after a large release of serotonin.

Serotonergic neurons in mammals and other invertebrates are spontaneously active with firing frequencies about $1 \mathrm{~Hz}$ [8]. Therefore, spontaneous activity might also deplete the releasable pool, especially over longer time periods. Because ChR2 activation can directly depolarize neurons, ChR2-mediated stimulation is not dependent on action potential propagation [24] and not reduced by TTX. Instead, TTX increases evoked release, demonstrating that spontaneous activity depletes some of the releasable serotonin pool. While inhibition of spontaneous serotonin release may decrease the baseline serotonin concentration, which would increase the peak height of stimulated release, this effect is likely to be small because basal levels are only about 5$10 \mathrm{nM}[25]$.

Increased release after TTX could result from an increase in total neuronal serotonin content or reallocation of serotonin to the releasable pool. Neuronal staining intensity, and thus neuronal serotonin content, was not significantly different for VNCs incubated in TTX and buffer control samples. Therefore, the data are more consistent with a reallocation of the releasable pool, possibly due to accumulation of primed vesicles at release sites. Indeed, data from multiple stimulation experiments showed that peak height in TTX-incubated VNCs started out larger but by the $4^{\text {th }}$ stimulation had decayed to a level similar to those incubated in buffer.

\section{ii. Reuptake is important for short-term refilling of the releasable pool}

Because SERT is a common therapeutic target, its role in the regulation of serotonin levels has been the subject of considerable investigation [26]. Our experiments 
confirm that, similar to other organisms, serotonin clearance in the fly was prolonged after transporter inhibition, consistent with the key role of reuptake in serotonin clearance [13]. Staining showed that neuronal serotonin content decreased by a third, but this depletion was not large enough to significantly affect the size of the releasable serotonin pool on the initial stimulation. When the cocaine-incubated VNCs were stressed with repeated stimulations, the peak height decayed faster than buffer-incubated controls. Refilling of the pool after cocaine was time dependent, with less depletion evident as inter-stimulation time increased. Therefore, SERT activity is necessary to maintain the releasable pool, especially on the 1-2 minute time scale. Decreased serotonin release per stimulation has also been observed in SERT knockout mice and rats [27, 28], although the results are more difficult to interpret because of the chronic nature of the inhibition.

Release in cocaine incubated VNCs was less than buffer-incubated VNCs, raising the question of autoregulatory feedback. Although a serotonin autoreceptor has not yet been identified in the fly, the application of large amounts of exogenous serotonin causes retraction of serotonergic projections [22]. However, retraction has not been observed after cocaine incubation (Condron, personal communication), suggesting that physiologically relevant changes in serotonin levels may not be adequate to induce morphological changes. While autoregulatory feedback could lower stimulated release, the decrease in staining intensity suggests that depletion rather than reallocation of serotonin is responsible for observed decreases in peak height.

\section{iii. Serotonin synthesis is critical to maintaining the releasable pool}

SERT knockout mice exhibit elevated synthesis rates, likely an adaptation to decreased reuptake [28]. We therefore hypothesized that synthesis would be necessary to maintain the releasable pool, especially concurrent with transporter inhibition. As 
predicted, the Tph inhibitor PCPA decreased staining intensity by a third and resulted in a trend towards decreased evoked release on the first stimulation. Synthesis is therefore important to maintaining the pool during spontaneous as well as evoked activity. The decay of peak height depended only on the number of stimulations, and not on the interval, suggesting that serotonin recycling is insufficient to maintain the pool regardless of time. It is unclear if lost serotonin diffuses out of the VNC or is otherwise broken down via acetylation [29].

The combination of synthesis and transporter inhibition produced even greater depletion. Incubation in both cocaine and PCPA decreased staining intensity by half and resulted in a 3-fold decrease in initial stimulated release. Similar decreases in staining intensity have been observed in Tph2 hypomorph flies after fluoxetine feeding; therefore depletion can also occur chronically [30]. The large decrease in initial peak height suggested serotonin depletion occurred through endogenous activity and indeed, concurrent TTX administration increased the initial peak to normal levels. Neuronal content was partially restored, to $80 \%$ of control, indicating that some serotonin was still lost, possibly through non-action potential mediated release or degradation. The substantial serotonin depletion observed with PCPA and cocaine highlights the critical nature of synthesis to maintain release during SSRI treatment.

Figure 10 shows a summary diagram of the mechanisms that contribute to the replenishment and depletion of the releasable pool of serotonin. Two sources for serotonin to be released are newly synthesized serotonin and serotonin that has been recycled via SERT. On a shorter time scale, such as $1 \mathrm{~min}$. stimulations, reuptake is responsible for maintaining about half the releasable pool after 4 stimulations while synthesis maintains about a third. On a longer time scale, with 10 min intervals, synthesis has the same contribution, a third, while reuptake is relatively less important, responsible for about a fifth of the pool. Therefore reuptake is important for the rapid 
replenishment of the releasable pool and synthesis is relatively more important for longer-term maintenance of the releasable pool. Additionally, the TTX studies show that endogenous activity depletes the releasable pool by about a quarter. Combining synthesis and reuptake inhibition severely depletes the releasable pool, and no serotonin is detectable after 5 stimulations, indicating that during reuptake inhibition synthesis is critical to maintaining release.

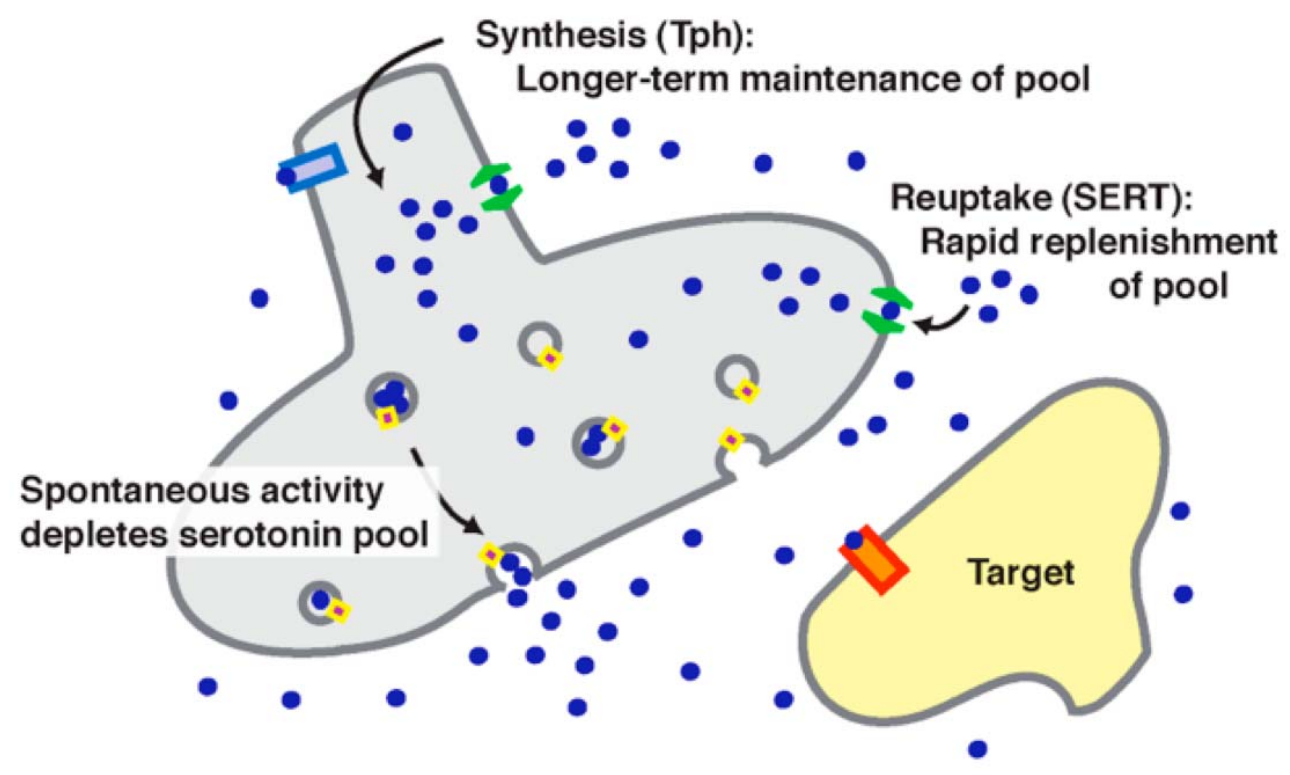

Figure 10. Diagram of mechanisms regulating the serotonin pool. Reuptake is most important for the short term replenishment (1-2 min. time scale) of the releasable pool. Synthesis is more important for longer term maintenance of the releasable pool. Spontaneous activity depletes the amount of serotonin available for evoked release.

\section{iv. Serotonin synthesis and SSRIS}

Although controversial, serotonin synthesis has previously been implicated in the pathophysiology of depression. Tph2 polymorphisms conferring decreased expression have been associated with suicidal behavior [3] and low levels of the serotonin precursor tryptophan have been found in depressed individuals [31]. In rodents, increases in synthesis are observed after SSRI administration [32] and their onset correlates with the 
onset of anti-depressant effect [33]. Furthermore, mice possessing a defective Tph2 allele, conferring a $50 \%$ decrease in enzyme expression, show decreased responses to antidepressants that can be restored by boosting serotonin synthesis with tryptophan $[14,34]$. In humans, SSRI efficacy can also be improved by tryptophan administration and patients that respond to SSRIs experience a relapse of depressive symptoms after administration of PCPA or acute tryptophan depletion [35, 36].

Our results suggest a physiological interpretation of behavioral data that show a dependence of SSRI treatment on synthesis. Through direct measurement of serotonin dynamics in the Drosophila, we show that synthesis is required to maintain the releasable serotonin pool, especially in the presence of reuptake inhibitors. Consequently, administering reuptake inhibitors to animals with deficient synthesis would further deplete serotonin pools leading to less, not greater, serotonin release. We propose that in the absence of elevated synthesis rates, serotonergic neurons cannot maintain their releasable pool after SSRI treatment and therefore serotonin levels would not be elevated and the desired therapeutic response not achieved. The efficacy of SSRIs may depend on the ability to upregulate synthesis. Exploring treatments that increase synthesis, along with reuptake inhibition, might be a productive line of study for depression treatment in humans. The characterization of Drosophila serotonergic signaling made possible by our method will facilitate future genetic screens to identify genetic elements critical for Tph or SERT function and their impact on serotonin release. Combined with pharmacological manipulations, such screens would represent a key step towards a pharmacogenetic approach for the treatment of psychiatric illness. 


\section{References:}

1. Murphy, D.L., A. Lerner, G. Rudnick, and K.P. Lesch, Serotonin transporter: gene, genetic disorders, and pharmacogenetics. Mol Interv, 2004. 4(2): p. 10923.

2. Lasky-Su, J.A., S.V. Faraone, S.J. Glatt, and M.T. Tsuang, Meta-analysis of the association between two polymorphisms in the serotonin transporter gene and affective disorders. Am J Med Genet B Neuropsychiatr Genet, 2005. 133(1): p. 110-5.

3. Li, D. and L. He, Meta-analysis supports association between serotonin transporter (5-HTT) and suicidal behavior. Mol Psychiatry, 2006. 12(1): p. 47-54.

4. Bauer, M., T. Bschor, A. Pfennig, P.C. Whybrow, J. Angst, M. Versiani, and H.J. Moller, World Federation of Societies of Biological Psychiatry (WFSBP) Guidelines for Biological Treatment of Unipolar Depressive Disorders in Primary Care. World J Biol Psychiatry, 2007. 8(2): p. 67-104.

5. Smits, K.M., L.J.M. Smits, J.S.A.G. Schouten, F.P.M.L. Peeters, and M.H. Prins, Does pretreatment testing for serotonin transporter polymorphisms lead to earlier effects of drug treatment in patients with major depression? A decision-analytic model. Clinical Therapeutics, 2007. 29(4): p. 691-702.

6. Coleman, C.M. and W.S. Neckameyer, Serotonin synthesis by two distinct enzymes in Drosophila melanogaster. Arch Insect Biochem Physiol, 2005. 59(1): p. 12-31.

7. Ma, P.M., B.S. Beltz, and E.A. Kravitz, Serotonin-containing neurons in lobsters: their role as gain-setters in postural control mechanisms. J Neurophysiol, 1992. 68(1): p. 36-54.

8. Zhang, Q.J., R. Gao, J. Liu, Y.P. Liu, and S. Wang, Changes in the firing activity of serotonergic neurons in the dorsal raphe nucleus in a rat model of Parkinsonos disease. Sheng Li Xue Bao, 2007. 59(2): p. 183-189.

9. Haghighi, F., et al., Genetic architecture of the human tryptophan hydroxylase 2 Gene: existence of neural isoforms and relevance for major depression. Mol Psychiatry, 2008.

10. Hen, R., Structural and functional conservation of serotonin receptors throughout evolution. Exs, 1993. 63: p. 266-78.

11. Bier, E., Drosophila, the golden bug, emerges as a tool for human genetics. Nat Rev Genet, 2005. 6(1): p. 9-23. 
12. Porzgen, P., S.K. Park, J. Hirsh, M.S. Sonders, and S.G. Amara, The Antidepressant-Sensitive Dopamine Transporter in Drosophila melanogaster: A Primordial Carrier for Catecholamines. Molecular Pharmacology, 2001. 59(1): p. 83-95.

13. Borue, X., S. Cooper, J. Hirsh, B. Condron, and B.J. Venton, Quantitative evaluation of serotonin release and clearance in Drosophila. J Neurosci Methods, 2009. 179(2): p. 300-8.

14. Cervo, L., et al., Genotype-dependent activity of tryptophan hydroxylase-2 determines the response to citalopram in a mouse model of depression. $\mathrm{J}$ Neurosci, 2005. 25(36): p. 8165-72.

15. Chen, J. and B.G. Condron, Branch architecture of the fly larval abdominal serotonergic neurons. Dev Biol, 2008. 320(1): p. 30-8.

16. Venton, B.J., et al., Cocaine Increases Dopamine Release by Mobilization of a Synapsin-Dependent Reserve Pool. Journal of Neuroscience, 2006. 26(12): p. 3206-3209.

17. Heien, M.L.A.V., P.E.M. Phillips, G.D. Stuber, A.T. Seipel, and R.M. Wightman, Overoxidation of carbon-fiber microelectrodes enhances dopamine adsorption and increases sensitivity. The Analyst, 2003. 128(12): p. 1413-1419.

18. Jackson, B.P., S.M. Dietz, and R.M. Wightman, Fast-scan cyclic voltammetry of 5-hydroxytryptamine. Anal Chem, 1995. 67(6): p. 1115-20.

19. Heien, M.L., M.A. Johnson, and R.M. Wightman, Resolving Neurotransmitters Detected by Fast-Scan Cyclic Voltammetry. Anal. Chem., 2004. 76(19): p. 56975704.

20. Zhang, X., J.M. Beaulieu, T.D. Sotnikova, R.R. Gainetdinov, and M.G. Caron, Tryptophan hydroxylase-2 controls brain serotonin synthesis. Science, 2004. 305(5681): p. 217.

21. Bunin, M.A. and R.M. Wightman, Quantitative evaluation of 5-hydroxytryptamine (serotonin) neuronal release and uptake: an investigation of extrasynaptic transmission. J Neurosci, 1998. 18(13): p. 4854-60.

22. Sykes, P.A. and B.G. Condron, Development and sensitivity to serotonin of Drosophila serotonergic varicosities in the central nervous system. Dev Biol, 2005. 286(1): p. 207-16.

23. Weilburg, J.B., An overview of SSRI and SNRI therapies for depression. Manag Care, 2004. 13(6 Suppl Depression): p. 25-33. 
24. Nagel, G., et al., Channelrhodopsin-2, a directly light-gated cation-selective membrane channel. Proc Natl Acad Sci U S A, 2003. 100(24): p. 13940-5.

25. Yoshitake, T., K. Fujino, J. Kehr, J. Ishida, H. Nohta, and M. Yamaguchi, Simultaneous determination of norepinephrine, serotonin, and 5-hydroxyindole-3acetic acid in microdialysis samples from rat brain by microbore column liquid chromatography with fluorescence detection following derivatization with benzylamine. Anal Biochem, 2003. 312(2): p. 125-33.

26. Perona, M.T., et al., Animal models of depression in dopamine, serotonin, and norepinephrine transporter knockout mice: prominent effects of dopamine transporter deletions. Behav Pharmacol, 2008. 19(5-6): p. 566-74.

27. Homberg, J.R., et al., Characterization of the serotonin transporter knockout rat: a selective change in the functioning of the serotonergic system. Neuroscience, 2007. 146(4): p. 1662-76.

28. Kim, D.K., et al., Altered serotonin synthesis, turnover and dynamic regulation in multiple brain regions of mice lacking the serotonin transporter.

Neuropharmacology, 2005. 49(6): p. 798-810.

29. Paxon, T.L., P.R. Powell, H.G. Lee, K.A. Han, and A.G. Ewing, Microcolumn separation of amine metabolites in the fruit fly. Anal Chem, 2005. 77(16): p. 5349-55.

30. Neckameyer, W.S., C.M. Coleman, S. Eadie, and S.F. Goodwin, Compartmentalization of neuronal and peripheral serotonin synthesis in Drosophila melanogaster. Genes, Brain and Behavior, 2007. 6(8): p. 756-769.

31. Cowen, P.J., M. Parry-Billings, and E.A. Newsholme, Decreased plasma tryptophan levels in major depression. J Affect Disord, 1989. 16(1): p. 27-31.

32. Kim, S.W., S.Y. Park, and O. Hwang, Up-regulation of tryptophan hydroxylase expression and serotonin synthesis by sertraline. Mol Pharmacol, 2002. 61(4): p. 778-85.

33. Shishkina, G.T., T.S. Kalinina, and N.N. Dygalo, Up-regulation of tryptophan hydroxylase-2 mRNA in the rat brain by chronic fluoxetine treatment correlates with its antidepressant effect. Neuroscience, 2007. 150(2): p. 404-12.

34. Invernizzi, R.W., Role of TPH-2 in brain function: news from behavioral and pharmacologic studies. J Neurosci Res, 2007. 85(14): p. 3030-5.

35. Delgado, P.L., D.S. Charney, L.H. Price, G.K. Aghajanian, H. Landis, and G.R. Heninger, Serotonin function and the mechanism of antidepressant action. 
Reversal of antidepressant-induced remission by rapid depletion of plasma tryptophan. Arch Gen Psychiatry, 1990. 47(5): p. 411-8.

36. Friedman, E., B. Shopsin, and S. Gershon, Effects of tranylcypromine on 5-HT uptake and its interaction with PCPA on rat brain 5-HT. Res Commun Chem Pathol Pharmacol, 1976. 15(1): p. 191-4. 


\section{Chapter 5. General discussion}

Serotonergic signaling has been implicated in a number of disorders including major depressive disorder (MDD). Based on the serotonin hypothesis, which links decreased serotonergic activity to depression, a goal of modern treatment is to elevate serotonin levels [1]. Because of the complexity of the serotonergic system, many aspects of its normal physiology and role in depression remain unclear. This dissertation has focused on developing a method to detect changes in extracellular serotonin in larval Drosophila and using this method to probe the basic mechanisms of serotonergic signaling. First, fast scan cyclic voltammetry (FSCV) at inserted microelectrodes was optimized for use in the fly. Next, a genetic approach was used to elicit release of endogenous serotonin, allowing a characterization of serotonin release and clearance in the larval ventral nerve cord (VNC). Finally, using multiple stimulations and pharmacological manipulation, we explored the mechanisms by which serotonergic neurons maintain their pool of releasable serotonin.

\section{The technical challenges of monoamine detection in the fly}

Drosophila offers many advantages to work in mammals, including a wellcharacterized, simple nervous system, short life cycle, completed genomic sequence [2], and ease of molecular and genetic manipulation [3, 4]. Flies cost significantly less than vertebrates to house, can be kept in much larger numbers thereby facilitating large-scale screens, and provide a good opportunity to investigate problems in a lower order animal prior to conducting experiments on mammals. However, the utility of the fly for the study of serotonergic signaling had been hampered by a lack of analytical tools. This section covers some of the challenges of detecting serotonin release and reuptake in the fly and how they have influenced the methodology developed. 


\section{i. FSCV: Technical considerations}

The main technical difficulty in working with Drosophila is size. The larval fly CNS is only $8 \mathrm{nl}$ in volume. Previous methods required the homogenization of the entire CNS to quantify serotonin levels, which gave little temporal or spatial information $[5,6]$. This is the first method available for the fly that allowed for the measurement of serotonin dynamics in single larva. Carbon fiber microelectrodes, at $50 \mu \mathrm{m}$ long by $7 \mu \mathrm{m}$ wide, were small enough to insert into the tiny CNS. FSCV measurements were collected every 100 ms, allowing rapid detection of release and clearance [7]. Because the electrode sampled from the extracellular space, measurements ascertained the functional fraction of total tissue serotonin content. FSCV provided a cyclic voltammogram (CV) which is characteristic of the analyte detected and therefore aided in its identification [8]. Although FSCV has many advantages, some concerns arise as well and are discussed below.

A chief concern with direct measurement of neurotransmitters is probe-induced tissue damage. The carbon fiber microelectrodes typically used for FSCV are much smaller than dialysis probes and consequently produce less damage [9]. Carbon itself is relatively inert and induces only minor fibrosis over the course of long-term implantation [10] and minimal short term reaction. Damage from microelectrodes, typically $7 \mu \mathrm{m}$ in diameter, is confined to $4 \mu \mathrm{m}$ away from the tip [9], as compared to $\sim 1.4 \mathrm{~mm}$ from the much larger dialysis probes [11]. A small, $2.5 \mu \mathrm{m}$ wide pool of extracellular fluid tends to accumulate at the electrode tip. The composition of the pool is unclear but likely remains close to that of the normal extracellular fluid [12]. A significant effort has been made to miniaturize the electrode in order to minimize tissue damage and refine signal localization. Tip diameters as small as $100 \mathrm{~nm}$ have been utilized in cell culture and in the future may be used to allow better localization and decreased damage in vivo [13]. 
Another concern is the disruption of endogenous signaling due to applied voltage and neuronal damage due to oxidized byproducts. Because of the small size of the electrode and voltage changes, the possible area impacted by applied voltage is quite small. The effect of FSCV on overall neuronal activity is therefore likely to be negligible [14]. In FSCV, oxidized monoamines are formed only briefly before being reduced again and represent a small percentage of the overall neurotransmitter concentration. This is different from amperometry, for instance, that can oxidize all neurotransmitter diffusing to its surface. Therefore, neurotransmitter levels are not impacted by measurement and neuronal exposure to oxidized byproducts is minimal.

A special issue when detecting serotonin is the gradual fouling of electrodes by oxidized serotonin that polymerizes and adsorbs to the electrode surface. FSCV minimizes the amount of time that serotonin remains oxidized but some can escape reduction. Modification of the waveform and holding potential to reduce the amount of time the electrode spends at potentials negative enough to allow the adsorption of oxidized serotonin of the electrode further reduces electrode fouling. Other substances present in the tissue can also adsorb to the electrode, reducing its sensitivity [15] and response time [16]. However, despite rapid initial decreases in sensitivity observed during electrode implantation, signals quickly stabilize and additional degradation proceeds slowly and in an activity-dependent manner. Therefore FSCV is well suited for detecting serotonin in tissue.

A final concern is the conversion of measured current to analyte concentrations, which depends on electrode calibration. Despite advances in electrode selectivity and stability, precise calibrations of in vivo signals are still difficult to obtain. Microdialysis experiments performed simultaneously with FSCV measurements show that values fall in between those calculated from pre and post-calibration of electrodes [17]. A major caveat of these experiments is that calibration of microdialysis data is also difficult. For 
consistency and ease of comparison to previous studies, this work used post-calibration to convert electrode-derived signals into neurotransmitter concentrations. Although precise estimation of actual neurotransmitter concentrations are not feasible due to calibration concerns, FSCV still offers very good estimates of their overall scale.

\section{ii. Stimulating serotonin release in the fly}

FSCV is best suited for measuring rapid changes in extracellular monoamine levels. Changes that occur more gradually, such as the diffusion of bath-applied dopamine described in Ch. 2, are prone to considerable error due to background drift. Two approaches are commonly used to circumvent this problem in vertebrates, microinjection of large amounts of neurotransmitter close to the microelectrode and electrical stimulation. Recently FSCV has also been used to monitor behaviorally mediated changes in endogenous signaling within freely moving rats but this approach necessitates attaching the electrode to the organism and is not feasible for the tiny fruit fly.

Electrical stimulation has been a preferred method to elicit the release of endogenous neurotransmitter. An electrical pulse is applied to an area of the tissue to spark action potential propagation and subsequent monoamine release. Electrical stimulation is non-specific, depolarizing all neurons within its range. Therefore a mixture of neurotransmitters is likely to be released, raising concerns about the downstream effect of this multi-molecule signal as well as the feasibility of distinguishing the signal from the target neurotransmitter from that concurrently released molecules. In brain slices, exogenous $5-\mathrm{HT}$ application is used as an alternative to non-specific electrical depolarization. However, the concentration utilized is over 10x that of the physiological range and time course of signal decay occurs over minutes [18]. While this is faster than 
the signals observed after bath application of dopamine, they are still prone to errors and do not provide any information about endogenous serotonin dynamics.

During our initial work with modifying FSCV for use in Drosophila, we explored the use of electrical stimulation. However, even with modification, stimulating electrodes were larger than the entire larval CNS, which meant that stimulation would be global. As an alternative, we explored genetic options to induce monoamine release. A recent development was the discovery of channelrhodopsin2 (ChR2), a blue light activated cation channel from green algae. ChR2 expression had been shown to provide single action potential control of neuronal signaling and had been placed under UAS control for use in the fly $[19,20]$. Expression of ChR2 allowed neuronal type-specific depolarization [21], therefore avoiding the issue of detecting serotonin amongst a mixture of other neurotransmitters. The ability to target the expression of constructs such as ChR2 to specific neuronal populations represents a key strength of the fly, access to an array of powerful genetic tools. ChR2 expression was also useful because it allowed us to explore physiologically relevant signals and evaluate the size of the releasable pool of serotonin.

\section{Serotonin signaling in the fly is analogous to that of mammals}

The initial characterization of serotonin release focused on definitively identifying the observed signal as serotonin, obtaining a dose-response curve for stimulated release, and estimating the Michaelis-Menten (M-M) constants for serotonin clearance in the fly. Characterization of serotonin release and clearance is important for comparing serotonergic signaling in the fly to that of mammals.

The identity of the signal was confirmed to be serotonin electrochemically and pharmacologically. Background-subtracted CVs obtained after 10s of blue light stimulation in a fly expressing ChR2 in serotonergic neurons were compared to those 
obtained during electrode calibration with $1 \mu \mathrm{M}$ serotonin. By observation, the CVs were similar in shape, identifying the detected analyte as serotonin. A high correlation coefficient, with $R^{2}$ values generally over 0.8 , was obtained by numerical comparison of the CVs, further supporting analyte identification. When the packaging of serotonin into vesicles was inhibited with the drug reserpine, the evoked release was not detected and the signal CV did not exhibit serotonin-specific peaks. When the drug PCPA was used to inhibit the rate-limiting enzyme in serotonin synthesis, tryptophan hydroxylase (Tph), the amount of signal detected with each stimulation decreased. The results of both experiments were consistent with the expectation that less serotonin would be released when serotonin synthesis or packaging is inhibited and therefore supported the assertion that the assay induced and detected serotonin release.

We detected serotonin concentrations ranging from 280 to $640 \mathrm{nM}$ following $2 \mathrm{~s}$ to 30 s of blue light exposure. These values are very close to those obtained by electrical stimulation in mammalian substantia nigra reticulata ( $\mathrm{SNr}$ ) slices, where values ranging from 54 to $640 \mathrm{nM}$ were detected for 1 to 20 electrical pulses [7]. Peak detected serotonin concentrations are likely to underestimate actual serotonin release due to concurrent reuptake [7]. Because elicited peak serotonin concentrations in mammals and fly are of comparable size, the size of their releasable serotonin pool is likely to be similar. Peak height decayed when stimulations were performed close together, suggesting that a sizeable portion of the releasable pool was mobilized during each stimulation. Our stimulations therefore likely release larger amounts of serotonin than would occur during spontaneous release.

Michaelis-Menten constants can be used to describe parameters for uptake. $V_{\max }$ is the maximal rate of uptake, which is dependent on the number of transporters. $\mathrm{K}_{\mathrm{T}}$ is the serotonin concentration at which clearance rate is half maximal and should be of the same order of magnitude as $\mathrm{K}_{\mathrm{m}}$. Our estimated $\mathrm{K}_{\mathrm{T}}$ for serotonin clearance in the fly is 
$350+/-80 \mathrm{nM}$ and this is close to previously published $\mathrm{K}_{\mathrm{m}}$ values of 490 and $637 \mathrm{nM}$ for Drosophila SERT expressed in transfected cells $[22,23]$. The estimated $V_{\max }$ of $170+/-$ $40 \mathrm{nM} / \mathrm{s}$, falls within the range of $K_{m}$ measurements in mammals, which is from $170 \mathrm{nM}$ [7] to $5.4 \mu \mathrm{M}$ [18]. While our kinetic parameters are just estimates, as the elicited peak heights are smaller than the concentration necessary to produce $V_{\max }$, they show that the parameters in the fly are of similar to those in mammals. The future directions section offers a discussion of a microinjection-based approach, which could help refine these estimates. In particular, exogenous application of a non-transported substance can allow clearance due to diffusion to be separated from that of transport. In keeping with previous literature, we estimated the Michaelis-Menten constants using the assumption that all clearance is due to reuptake. A more accurate estimate could be obtained by accounting for diffusion in the calculations.

\section{Reuptake clears serotonin from the extracellular space and recycled serotonin is rapidly reintegrated into the releasable pool}

Our initial characterization of serotonergic signaling highlighted its similarity to that of mammals. We therefore expected that like mammals, reuptake in the fly would play a key role in the clearance of extracellular serotonin. Although Drosophila SERT shares over $50 \%$ overall amino acid sequence identity with that of mammals [24], cell culture assays show that their drug affinities differ somewhat [23]. For example, in humans, cocaine shows nearly equal affinity for SERT and DAT, while in the fly cocaine has 6x greater affinity for SERT $[22,23]$. Despite a higher amount of promiscuity in drug affinity, Drosophila is very useful for pharmacological experiments. Consistent with our prediction, transporter blockers like cocaine and fluoxetine increased apparent $\mathrm{K}_{\mathrm{m}}$ over 4-fold. These changes were of similar magnitude to the 5-6 fold changes in $\mathrm{K}_{\mathrm{m}}$ observed in mammals [25]. Serotonin release in the fly is localized to a small region in the interior 
of the CNS, the neuropil, with the majority of cell bodies residing outside this area. With this morphology, diffusion may play a larger role in clearing serotonin released in the neuropil and therefore less difference in apparent $K_{m}$ may be observed after the inhibition of transport.

Serotonin taken back up via the transporter may be repackaged for future release, thereby recycling the neurotransmitter. We hypothesized that transporter inhibition would therefore interfere with serotonin recycling and lead to decreases in the releasable pool of serotonin that could be observed during multiple stimulation experiments. When stimulations were performed close together in the presence of a transporter blocker, serotonin release decreased significantly. Decreases in total serotonin suggested that depletion rather than a decrease in the size of the releasable pool due to autoregulatory feedback was responsible for the phenomenon. When stimulations were performed farther apart, less depletion was evident indicating that synthesis can compensate for inhibited reuptake when given enough time, at least 10 minutes in the fly. Altogether, this set of experiments showed that reuptake in the fly plays an analogous role to that in mammals. The results also implicated serotonin synthesis as a putative mechanism by which serotonergic neurons maintain their serotonin content.

\section{Serotonergic neurons maintain serotonin content through synthesis}

We explored the impact of acute synthesis inhibition on serotonergic neurons. Our experiments showed that synthesis is critical to replenishing the releasable pool. After 30 minutes of PCPA incubation, neuronal serotonin content decreases by nearly $40 \%$ and initial stimulated serotonin release decreases by $\sim 100 \mathrm{nM}$. Subsequent stimulations led to greater decreases in signal, regardless of the time between stimulations. Even after a 30 minute wait, the peak height did not recover indicating that 
reuptake is insufficient to fully compensate for decreased synthesis over this timeframe. Our results therefore suggested that an inhibited or defective serotonin synthesis mechanism would predispose the animal to serotonin depletion.

Synthesis is especially important when reuptake is concurrently inhibited. The effects of cocaine and PCPA were found to be additive and incubation in both drugs caused a 3- fold decrease in initial peak height and a 50\% decrease in total neuronal serotonin content. Inhibition of action potential generation rescued serotonin release and partially rescued total neuronal serotonin content, showing that endogenous activity is the primary mechanism by which serotonin depletion occurs. A small percentage of total serotonin is lost even when activity is inhibited. This could occur through serotonin metabolism or as a result of non-action potential mediated release. Although the lack of an identified MAO homologue had cast doubt whether serotonin metabolism occurs in the fly, a recent analysis of metabolic products in the fly suggests that serotonin is metabolized through acetylation [26]. Similar decreases in staining intensity have been observed in Tph2 hypomorph flies after fluoxetine feeding [27]. Therefore, the acute changes in neuronal serotonin content observed in our experiments after uptake and synthesis inhibition can persist chronically. These results could have important implications for our understanding of the changes that occur after SSRI administration, which are discussed in the next section.

Although it is widely accepted that the administration of synthesis inhibitors such as PCPA decreases serotonin levels, the putative link between serotonin levels, synthesis, and the pathophysiology of mental disorders remains controversial [28, 29]. Tph polymorphisms have not been conclusively associated with depression although a recent meta analysis suggests a link to suicide [30]. Low serotonin and serotonin metabolite content has been identified during post-mortem brain analysis of depressed and suicidal individuals [31]. Low plasma tryptophan levels, which can cause serotonin 
depletion, have also been observed in depressed individuals [32]. Although decreased serotonin is commonly observed during depression, it remains unclear if it is a cause, outcome, or byproduct of the disorder.

Evidence on causality in the relationship between serotonin availability and depression is mixed. When synthesis is inhibited in rats, they exhibit impaired performance on behavioral tasks typically enhanced by SSRI administration [33] and appear to undergo a cognitive decline similar to that sometimes observed in patients with MDD [34]. Administration of interferons, which deplete tryptophan and increase serotonin catabolism, can induce symptoms of depression in some patients, suggesting that in some situations decreasing serotonin levels can induce mental illness $[35,36]$. Conversely, acute tryptophan depletion in individuals without a clinical or family history of depression does not induce the development of depressive symptoms and only minimally influences the interpretation of emotional stimuli [37-39]. Greater effects on emotional processing, specifically the introduction of a positive bias, are observed in the converse experiment where tryptophan supplementation is used to increase serotonin availability [40]. Therefore serotonin synthesis and availability may play an indirect role in the development of depression but may be important for other aspects such as memory or emotional processing.

\section{Serotonin synthesis and the treatment of depression}

Recent studies directly implicate serotonin synthesis in the clinical effects of SSRI treatment [41]. Depressed patients, who had been successfully treated with SSRIs, can experience a relapse of symptoms after administration of PCPA or acute tryptophan depletion $[42,43]$. Tph polymorphisms in humans have been associated with the specificity of therapeutic response to SSRI treatment $[44,45]$. Mice possessing a defective Tph2 allele, conferring a 50\% decrease in enzyme expression, also show 
decreased responses to antidepressants that can be restored by boosting serotonin synthesis with tryptophan administration [46, 47]. Our results show that in the fly, concurrent decreases in synthesis and reuptake deplete neuronal serotonin content. We propose that in the absence of elevated synthesis rates, serotonergic neurons cannot maintain their releasable pool and therefore serotonin levels cannot be elevated.

In rats, semi-chronic (8-14 days) SSRI administration decreases brainstem Tph2 mRNA levels [48] and leads to overall reductions in serotonin and its metabolite 5-HIAA $[49,50]$. During this initial period of SSRI exposure, rats exhibit an increase in anxiety during the plus-maze test [51]. A similar increase in anxiety has been observed in some patients during the initial stages of SSRI treatment [52]. Although a direct link between behavior and physiology is difficult to make, these studies suggest that decreased serotonin levels may be responsible for anxiety-like behavior. After the initial 2 week period on SSRIs, serotonin synthesis increases to above normal levels [53] and this increase correlates temporally with the onset of anti-depressant behavioral effects and disappearance of anxiety symptoms [54]. Our results suggest that the releasable pool of serotonin may remain quite small during the initial stage of SSRI treatment with serotonin availability increasing only after synthesis is upregulated. It may therefore be therapeutically useful to add greater support for serotonin synthesis during the initial stage of SSRI treatment by increasing precursor availability.

Surprisingly few clinical trials have been performed to assess the utility of synthesis-enhancing drugs in the treatment of MDD and most of those were conducted at least 20 years ago [1]. A recent meta-analysis of serotonin precursor supplementation revealed only a small number of well-controlled studies and even these were too small to reach an adequate conclusion. A stumbling block has been that 5-HTP and L-tryptophan fall under the supplements umbrella in the USA and are consequently not subject to the same regulations or stringent testing procedures as pharmaceuticals. A potential 
consequence of this lack of regulation was seen in the 1990s when contaminated Ltryptophan led to an epidemic of eosinophillia myalgia syndrome resulting in at least 38 deaths $[55,56]$. Another possible concern has been the induction of serotonin syndrome. However, no cases of serotonin syndrome have thus far been reported in association with synthesis augmentation protocols alone or in combination with SSRIs or MAO inhibitors $[57,58]$. Since the half-life of tryptophan and 5-HTP in the bloodstream is relatively short (4h) and higher peak concentrations have been associated with higher rates of gastrointestinal side effects, the development of a gradual-release preparation should improve the efficacy and tolerability of these supplements [1]. Furthermore, the use of carbidopa to block peripheral conversion could further reduce side effects by allowing the amino acids to be preferentially targeted to the CNS.

Because of safety concerns and a lack of ability to file for patents, the pharmaceutical industry has thus-far shown little interest in synthesis-enhancing drugs. The results of my thesis combined with those from clinical and rodent studies suggest that we should reexamine the clinical utility of these drugs. Augmenting serotonin synthesis may be useful for achieving remission of depressive symptoms in a greater number of patients and in alleviating initial anxiety during SSRI treatment.

\section{Future directions}

\section{i. Chronic SERT inhibition - modeling SSRI treatment}

The mechanisms underlying the therapeutic actions of SSRIs are still subject to considerable debate [59-61]. Of special interest is the delay between the pharmacologic effects of SSRIs, which occur as soon as the brain is exposed to the drug, and the therapeutic effects, which can take weeks. One proposed explanation for this phenomenon is that time must pass for autoreceptors to desensitize [62]. During the initial stages of treatment, autoregulatory feedback reduces serotonin synthesis and 
neuron activity and only after the feedback loop desensitizes can levels rise enough to be therapeutic. The rapid life cycle of Drosophila provides the opportunity to study chronic effects of SERT inhibition, a situation analogous to that which occurs during SSRI treatment in adults, within a short time frame, 2-3 days. Genetic manipulations can be utilized to probe the basic mechanisms of tissue response to acute or chronic SERT inhibition. Although a serotonin autoreceptor has not yet been characterized in the fly, the application of large amounts of exogenous serotonin has been shown to elicit the retraction of serotonergic projections [63]. Furthermore, blocking serotonergic neuron activity leads to an overgrowth of serotonergic projections, suggesting that on a timecourse of days the serotonergic system can adapt to activity-related changes.

Another aspect of serotonergic signaling that can be explored using Drosophila is the developmental effect of altered serotonin levels. Serotonergic neurons develop very early, sending projections throughout the brain, and therefore are in a prime position to modulate the maturation of other neuronal circuits. The effect of serotonin signaling disruption during development has been an area of significant research [64-72]. Work in mouse and rat models has uncovered potential differences in the effect of and sensitivity to SERT inhibition across development, however the phenotypes are complex and there is little temporal control over the SERT depletion. Potential developmental effects of increased serotonin levels can be explored by comparing larvae lacking SERT to those exposed to pharmacological manipulation during later development and those exposed to it acutely as mature larvae. Furthermore, the genetic tools in Drosophila allow one to isolate SERT expression temporally based on temperature-sensitive expression constructs. Using these constructs, the effects of early developmental SERT inhibition can be compared with those of late to better understand which processes are most affected. 


\section{ii. Development of exogenously applied serotonin clearance assay}

The use of $\mathrm{ChR} 2$ to induce serotonin release has the advantage of being able to probe endogenous serotonin pools. However, this approach introduces several constraints on the study of serotonin clearance. First, the use of genetic constructs necessitates that every experimental animal be genetically modified to express them. This greatly complicates possible genetic screens. Second, maximal stimulation elicits much less serotonin than would be required to reach $\mathrm{V}_{\max }$ and properly measure it. Finally, to measure the effect of diffusion, it is useful to introduce a molecule that is not transported and measure its clearance. This is difficult to achieve with the current methodology. For these reasons, the development of a microinjection-based exogenous serotonin application protocol will be very useful for the study of serotonin clearance.

This approach is best be accomplished by the insertion of a microinjection pipette 2-3 segments away from the detection electrode. Micro-pipettes have been routinely used for applications as small as patching single cells and are therefore easily made small enough to insert into the fly VNC. The chief concern will be delivering a large enough concentration of serotonin in the smallest volume possible to minimize fluid bolus-related distortion of the tissue. The initial characterization of this method will include the application of an electroactive molecule that is not transported, such as acetaminophen, to measure the rate of clearance by diffusion. Combined with subsequent experiments using increasing serotonin concentrations to produce peaks lager than $4 \mu \mathrm{M}$, these initial experiments will allow a much more accurate estimation of the Michaelis-Menten constants for serotonin uptake in the larval fly. Unlike previous experiments that assumed all clearance was due to transport, the results of these experiments would allow transport to be separated from non-transport mechanisms. They would also provide the first precise, real-time measure of diffusion in the fly VNC. 
Subsequent work using this method should focus on using it as a readout in screens for genetic elements affecting serotonin clearance. The J. Hirsh laboratory here at the University of Virginia has created many mutations in SERT and its promotor and our assay can be used to determine which mutations affect clearance. Additional assays could be performed in the presence of a transporter inhibitor to look for mutations that render SERT resistant to pharmacological inhibition. One such mutation has already been putatively identified on the basis of a lack of behavioral response to cocaine ( $\mathrm{J}$. Hirsh, private communication). The screen can also be broadened to include potential interacting genetic elements elsewhere in the genome. Once identified in a screen using exogenous serotonin application, interesting mutations could be subsequently recombined with ChR2 constructs to explore the mutation's effect on serotonin release. Therefore, in combination, these assays would greatly expand the utility of the fly for genetic and pharmacogenetic screens.

\section{iii. The characterization of dopamine release and clearance in larval Drosophila}

Dopamine has been implicated in many human behaviors, especially psychostimulant addiction. The loss of dopaminergic neurons is associated with the development of Parkinson's disease and dopamine receptors are the target of many antipsychotic drugs $[73,74]$. Like the serotonergic system, a great deal of homology between mammals and fly exists in the dopaminergic system. Recent work has shown that dopaminergic neurotransmission in the fly, like that in mammals, is responsible for the arousing effects of methamphetamines and has been implicated in that of caffeine [75]. Fly models of Parkinson's disease have been developed and exhibit agedependent degeneration of dopaminergic neurons with accompanying abnormalities in locomotor activity $[76,77]$. Assays in these flies have focused on neuronal morphology and behavior because a technique for direct measurement of dopamine signaling was 
not available. However, morphological assays may miss functional defects and behavioral phenotypes are not specific to dopaminergic dysfunction. For instance, DJ-1 mutants show severe locomotor defects, suggestive of dopamine dysfunction, in the absence of neuron degeneration [78].

The method developed in this dissertation is easily applicable to the study of dopamine release in clearance. Some preliminary work on detecting dopamine is presented in $\mathrm{Ch} 2$ and 3. This work would not only add to our knowledge of the basic mechanisms of dopaminergic signaling and how they are affected in disease states but also provide a paradigm in which dopaminergic and serotonergic signaling may be compared. Although these monoaminergic systems are very similar, they have been adapted to perform widely different tasks. Work in mammals suggests that some differences in basic mechanisms may exist, especially in relation to monoamine storage (R. M. Wightman, private communication). Additional work may also be done to explore putative serotonin-dopamine interactions.

\section{iv. Measuring monoamine release and clearance in adult Drosophila}

Although late L3 larvae have a mature nervous system that has been morphologically well characterized, the adult offers several advantages. The two main advantages are the potential to immobilize the CNS without needing to fully remove it from the organism and a larger repository of behaviors that can be linked to measurements. The Ewing group has recently published a study using FSCV to probe dopamine clearance after application of exogenous dopamine [79]. Their technique involves the immobilization of an adult fly using agarose. The exoskeleton overlying the brain is subsequently removed to expose it to electrode insertion. The exposed brain is bathed in a drop of buffer and dopamine is microinjected overtop of the region of the inserted electrode. This protocol could be adapted to monitor endogenous monoamine 
release and clearance in adult flies expressing ChR2. Because the brain is exposed, sufficient blue light should reach the neurons and induce depolarization like that observed in the larvae.

Data from adults could be compared to that from larvae to explore differences in serotonin release and clearance between the two stages. More importantly, flies of different ages can be tested. By comparing young to old flies, the effects of ageing on the monoaminergic systems can be explored. Furthermore, these experiments could be used to directly test for decaying monoamine function and to screen for novel treatments to alleviate it. Measurements could then be directly correlated with behavioral and morphological changes. Therefore, the expansion of FSCV to monitor monoaminergic signaling in the adult Drosophila would increase the utility of the fly for ageing and neurodegeneration research.

\section{Concluding remarks}

In summary, this dissertation describes the creation of a novel combination of methods to monitor changes in extracellular monoamine concentrations in the fruit fly larvae. Targeted channelrhodopsin2 expression allowed for specific depolarization of serotonergic or dopaminergic neurons. Resulting signals were detected with sub-second resolution using FSCV at carbon fiber microelectrodes. Using this technique, the first ever measurements of serotonin release and clearance in larval Drosophila were obtained. Using pharmacological manipulation combined with repeat stimulation experiments, the basic mechanisms of serotonin signaling were explored. Reuptake was shown to be critical to serotonin clearance and the rapid replenishment of the releasable pool of serotonin. Synthesis was critical for the longer-term replenishment of the releasable pool, especially when reuptake was concurrently inhibited. These results give insight into the possible effects of SSRIs on the serotonergic system and the 
important role that synthesis may play in this phenomenon and overall serotonergic neuron function. The have also paved the way for future use of the fly for large-scale genetic analysis of neurotransmitter dynamics. This dissertation was completed under the direction of Jill Venton, PhD, in the Department of Chemistry and Neuroscience Graduate Program. 


\section{References:}

1. Turner, E.H., J.M. Loftis, and A.D. Blackwell, Serotonin a la carte: supplementation with the serotonin precursor 5-hydroxytryptophan. Pharmacol Ther, 2006. 109(3): p. 325-38.

2. Adams, M.D., et al., The Genome Sequence of Drosophila melanogaster. Science, 2000. 287(5461): p. 2185-2195.

3. Brand, A.H. and E.-L. Dormand, The GAL4 system as a tool for unravelling the mysteries of the Drosophila nervous system. Current Opinion in Neurobiology, 1995. 5(5): p. 572-578.

4. Bier, E., Drosophila, the golden bug, emerges as a tool for human genetics. Nat Rev Genet, 2005. 6(1): p. 9-23.

5. Hardie, S.L. and J. Hirsh, An improved method for the separation and detection of biogenic amines in adult Drosophila brain extracts by high performance liquid chromatography. Journal of Neuroscience Methods, 2006. 153(2): p. 243-249.

6. Coleman, C.M. and W.S. Neckameyer, Substrate regulation of serotonin and dopamine synthesis in Drosophila. Invert Neurosci, 2004. 5(2): p. 85-96.

7. Bunin, M.A. and R.M. Wightman, Quantitative evaluation of 5-hydroxytryptamine (serotonin) neuronal release and uptake: an investigation of extrasynaptic transmission. J Neurosci, 1998. 18(13): p. 4854-60.

8. Garris, P.A., et al., A role for presynaptic mechanisms in the actions of nomifensine and haloperidol. Neuroscience, 2003. 118(3): p. 819-829.

9. Peters, J.L., L.H. Miner, A.C. Michael, and S.R. Sesack, Ultrastructure at carbon fiber microelectrode implantation sites after acute voltammetric measurements in the striatum of anesthetized rats. J Neurosci Methods, 2004. 137(1): p. 9-23.

10. Tayton, K., G. Phillips, and Z. Ralis, Long-term effects of carbon fibre on soft tissues. J Bone Joint Surg Br, 1982. 64(1): p. 112-4.

11. Clapp-Lilly, K.L., R.C. Roberts, L.K. Duffy, K.P. Irons, Y. Hu, and K.L. Drew, An ultrastructural analysis of tissue surrounding a microdialysis probe. J Neurosci Methods, 1999. 90(2): p. 129-42.

12. Koryta, J., Electrochenical polarization phenomena at the interface of two immiscible electrolyte solutions. Electrochemistry, 1979.

13. Wu, W.Z., et al., Monitoring dopamine release from single living vesicles with nanoelectrodes. J Am Chem Soc, 2005. 127(25): p. 8914-5. 
14. Armstrong, D.M. and T. Drew, Locomotor-related neuronal discharges in cat motor cortex compared with peripheral receptive fields and evoked movements. J Physiol, 1984. 346: p. 497-517.

15. Adams, R.N., In vivo electrochemical measurements in the CNS. Prog Neurobiol, 1990. 35(4): p. 297-311.

16. O'Neill, R.D. and J.P. Lowry, On the significance of brain extracellular uric acid detected with in-vivo monitoring techniques: a review. Behav Brain Res, 1995. 71(1-2): p. 33-49.

17. Logman, M.J., E.A. Budygin, R.R. Gainetdinov, and R.M. Wightman, Quantitation of in vivo measurements with carbon fiber microelectrodes. J Neurosci Methods, 2000. 95(2): p. 95-102.

18. Daws, L.C., S. Montanez, W.A. Owens, G.G. Gould, A. Frazer, G.M. Toney, and G.A. Gerhardt, Transport mechanisms governing serotonin clearance in vivo revealed by high-speed chronoamperometry. J Neurosci Methods, 2005. 143(1): p. 49-62.

19. Schroll, C., et al., Light-induced activation of distinct modulatory neurons triggers appetitive or aversive learning in Drosophila larvae. Curr Biol, 2006. 16(17): p. 1741-7.

20. Suh, G.S., S. Ben-Tabou de Leon, H. Tanimoto, A. Fiala, S. Benzer, and D.J. Anderson, Light activation of an innate olfactory avoidance response in Drosophila. Curr Biol, 2007. 17(10): p. 905-8.

21. Nagel, G., et al., Channelrhodopsin-2, a directly light-gated cation-selective membrane channel. Proc Natl Acad Sci U S A, 2003. 100(24): p. 13940-5.

22. Corey, J.L., M.W. Quick, N. Davidson, H.A. Lester, and J. Guastella, A cocainesensitive Drosophila serotonin transporter: cloning, expression, and electrophysiological characterization. Proc Natl Acad Sci U S A, 1994. 91(3): p. 1188-92.

23. Porzgen, P., S.K. Park, J. Hirsh, M.S. Sonders, and S.G. Amara, The Antidepressant-Sensitive Dopamine Transporter in Drosophila melanogaster: A Primordial Carrier for Catecholamines. Molecular Pharmacology, 2001. 59(1): p. 83-95.

24. Demchyshyn, L.L., et al., Cloning, expression, and localization of a chloridefacilitated, cocaine-sensitive serotonin transporter from Drosophila melanogaster. Proc Natl Acad Sci U S A, 1994. 91(11): p. 5158-62. 
25. John, C.E. and S.R. Jones, Voltammetric characterization of the effect of monoamine uptake inhibitors and releasers on dopamine and serotonin uptake in mouse caudate-putamen and substantia nigra slices. Neuropharmacology, 2007. 52(8): p. 1596-605.

26. Paxon, T.L., P.R. Powell, H.G. Lee, K.A. Han, and A.G. Ewing, Microcolumn separation of amine metabolites in the fruit fly. Anal Chem, 2005. 77(16): $p$. 5349-55.

27. Neckameyer, W.S., C.M. Coleman, S. Eadie, and S.F. Goodwin, Compartmentalization of neuronal and peripheral serotonin synthesis in Drosophila melanogaster. Genes, Brain and Behavior, 2007. 6(8): p. 756-769.

28. Cowen, P.J., Serotonin and depression: pathophysiological mechanism or marketing myth? Trends Pharmacol Sci, 2008. 29(9): p. 433-6.

29. Charney, D.S., Monoamine dysfunction and the pathophysiology and treatment of depression. J Clin Psychiatry, 1998. 59 Suppl 14: p. 11-4.

30. Li, D. and L. He, Meta-analysis supports association between serotonin transporter (5-HTT) and suicidal behavior. Mol Psychiatry, 2006. 12(1): p. 47-54.

31. Owens, M.J. and C.B. Nemeroff, Role of serotonin in the pathophysiology of depression: focus on the serotonin transporter. Clin Chem, 1994. 40(2): p. 28895.

32. Cowen, P.J., M. Parry-Billings, and E.A. Newsholme, Decreased plasma tryptophan levels in major depression. J Affect Disord, 1989. 16(1): p. 27-31.

33. Hritcu, L., M. Clicinschi, and T. Nabeshima, Brain serotonin depletion impairs short-term memory, but not long-term memory in rats. Physiol Behav, 2007. 91(5): p. 652-7.

34. Austin, M.P., P. Mitchell, and G.M. Goodwin, Cognitive deficits in depression: possible implications for functional neuropathology. Br J Psychiatry, 2001. 178: $\mathrm{p}$. 200-6.

35. Loftis, J.M. and P. Hauser, The phenomenology and treatment of interferoninduced depression. J Affect Disord, 2004. 82(2): p. 175-90.

36. Bonaccorso, S., V. Marino, M. Biondi, F. Grimaldi, F. Ippoliti, and M. Maes, Depression induced by treatment with interferon-alpha in patients affected by hepatitis C virus. J Affect Disord, 2002. 72(3): p. 237-41. 
37. Williams, J.H., D.I. Perrett, G.D. Waiter, and S. Pechey, Differential effects of tryptophan depletion on emotion processing according to face direction. Soc Cogn Affect Neurosci, 2007. 2(4): p. 264-273.

38. Moreno, F.A., et al., Tryptophan depletion and depressive vulnerability. Biol Psychiatry, 1999. 46(4): p. 498-505.

39. Benkelfat, C., M.A. Ellenbogen, P. Dean, R.M. Palmour, and S.N. Young, Moodlowering effect of tryptophan depletion. Enhanced susceptibility in young men at genetic risk for major affective disorders. Arch Gen Psychiatry, 1994. 51(9): p. 687-97.

40. Murphy, S.E., C. Longhitano, R.E. Ayres, P.J. Cowen, and C.J. Harmer, Tryptophan supplementation induces a positive bias in the processing of emotional material in healthy female volunteers. Psychopharmacology (Berl), 2006. 187(1): p. 121-30.

41. Cervo, L., et al., Genotype-dependent activity of tryptophan hydroxylase-2 determines the response to citalopram in a mouse model of depression. $\mathrm{J}$ Neurosci, 2005. 25(36): p. 8165-72.

42. Delgado, P.L., D.S. Charney, L.H. Price, G.K. Aghajanian, H. Landis, and G.R. Heninger, Serotonin function and the mechanism of antidepressant action. Reversal of antidepressant-induced remission by rapid depletion of plasma tryptophan. Arch Gen Psychiatry, 1990. 47(5): p. 411-8.

43. Friedman, E., B. Shopsin, and S. Gershon, Effects of tranylcypromine on 5-HT uptake and its interaction with PCPA on rat brain 5-HT. Res Commun Chem Pathol Pharmacol, 1976. 15(1): p. 191-4.

44. Peters, E.J., S.L. Slager, P.J. McGrath, J.A. Knowles, and S.P. Hamilton, Investigation of serotonin-related genes in antidepressant response. Mol Psychiatry, 2004. 9(9): p. 879-89.

45. Tzvetkov, M.V., J. Brockmoller, I. Roots, and J. Kirchheiner, Common genetic variations in human brain-specific tryptophan hydroxylase-2 and response to antidepressant treatment. Pharmacogenet Genomics, 2008. 18(6): p. 495-506.

46. Invernizzi, R.W., Role of TPH-2 in brain function: news from behavioral and pharmacologic studies. J Neurosci Res, 2007. 85(14): p. 3030-5.

47. Sakowski, S.A., T.J. Geddes, and D.M. Kuhn, Mouse tryptophan hydroxylase isoform 2 and the role of proline 447 in enzyme function. J Neurochem, 2006. 96(3): p. 758-65. 
48. Dygalo, N.N., G.T. Shishkina, T.S. Kalinina, A.M. Yudina, and E.S. Ovchinnikova, Effect of repeated treatment with fluoxetine on tryptophan hydroxylase-2 gene expression in the rat brainstem. Pharmacol Biochem Behav, 2006. 85(1): p. 2207.

49. Caccia, S., C. Fracasso, S. Garattini, G. Guiso, and S. Sarati, Effects of shortand long-term administration of fluoxetine on the monoamine content of rat brain. Neuropharmacology, 1992. 31(4): p. 343-7.

50. Muck-Seler, D., A. Jevric-Causevic, and M. Diksic, Influence of fluoxetine on regional serotonin synthesis in the rat brain. J Neurochem, 1996. 67(6): p. 243442.

51. Silva, R.C. and M.L. Brandao, Acute and chronic effects of gepirone and fluoxetine in rats tested in the elevated plus-maze: an ethological analysis. Pharmacol Biochem Behav, 2000. 65(2): p. 209-16.

52. Londborg, P.D., W.T. Smith, V. Glaudin, and J.R. Painter, Short-term cotherapy with clonazepam and fluoxetine: anxiety, sleep disturbance and core symptoms of depression. J Affect Disord, 2000. 61(1-2): p. 73-9.

53. Kim, S.W., S.Y. Park, and O. Hwang, Up-regulation of tryptophan hydroxylase expression and serotonin synthesis by sertraline. Mol Pharmacol, 2002. 61(4): p. 778-85.

54. Shishkina, G.T., T.S. Kalinina, and N.N. Dygalo, Up-regulation of tryptophan hydroxylase-2 mRNA in the rat brain by chronic fluoxetine treatment correlates with its antidepressant effect. Neuroscience, 2007. 150(2): p. 404-12.

55. Das, Y.T., M. Bagchi, D. Bagchi, and H.G. Preuss, Safety of 5-hydroxy-Ltryptophan. Toxicol Lett, 2004. 150(1): p. 111-22.

56. Michelson, D., et al., An eosinophilia-myalgia syndrome related disorder associated with exposure to L-5-hydroxytryptophan. J Rheumatol, 1994. 21(12): p. 2261-5.

57. Meltzer, H., B. Bastani, K. Jayathilake, and M. Maes, Fluoxetine, but not tricyclic antidepressants, potentiates the 5-hydroxytryptophan-mediated increase in plasma cortisol and prolactin secretion in subjects with major depression or with obsessive compulsive disorder. Neuropsychopharmacology, 1997. 17(1): p. 1-11.

58. Kline, N. and W. Sacks, Treatment of depression with an mao inhibitor followed by 5-HTP--an unfinished research project. Acta Psychiatr Scand Suppl, 1980.

280: p. 233-41. 
59. Kiss, J.P., Theory of active antidepressants: a nonsynaptic approach to the treatment of depression. Neurochem Int, 2008. 52(1-2): p. 34-9.

60. Marcussen, A.B., P. Flagstad, P.E. Kristjansen, F.F. Johansen, and U. Englund, Increase in neurogenesis and behavioural benefit after chronic fluoxetine treatment in Wistar rats. Acta Neurol Scand, 2008. 117(2): p. 94-100.

61. Owen, J.C. and P.S. Whitton, Chronic treatment with antidepressant drugs reversibly alters NMDA mediated regulation of extracellular 5-HT in rat frontal cortex. Brain Res Bull, 2006. 70(1): p. 62-7.

62. Butler, S.G. and M.J. Meegan, Recent developments in the design of antidepressive therapies: targeting the serotonin transporter. Curr Med Chem, 2008. 15(17): p. 1737-61.

63. Sykes, P.A. and B.G. Condron, Development and sensitivity to serotonin of Drosophila serotonergic varicosities in the central nervous system. Dev Biol, 2005. 286(1): p. 207-16.

64. Holmes, A., D.L. Murphy, and J.N. Crawley, Abnormal behavioral phenotypes of serotonin transporter knockout mice: parallels with human anxiety and depression. Biol Psychiatry, 2003. 54(10): p. 953-9.

65. Hohmann, C.F., E.M. Walker, C.B. Boylan, and M.E. Blue, Neonatal serotonin depletion alters behavioral responses to spatial change and novelty. Brain Research, 2007. 1139: p. 163-177.

66. Hohmann, C.F., C. Richardson, E. Pitts, and J. Berger-Sweeney, Neonatal 5, 7DHT lesions cause sex-specific changes in mouse cortical morphogenesis. Neural Plast, 2000. 7(4): p. 213-32.

67. Hansen, H.H., C. Sanchez, and E. Meier, Neonatal administration of the selective serotonin reuptake inhibitor Lu 10-134-C increases forced swimming-induced immobility in adult rats: a putative animal model of depression? J Pharmacol Exp Ther, 1997. 283(3): p. 1333-41.

68. Upton, A.L., N. Salichon, C. Lebrand, A. Ravary, R. Blakely, I. Seif, and P. Gaspar, Excess of serotonin (5-HT) alters the segregation of ispilateral and contralateral retinal projections in monoamine oxidase $A$ knock-out mice: possible role of 5-HT uptake in retinal ganglion cells during development. J Neurosci, 1999. 19(16): p. 7007-24. 
69. Salichon, N., et al., Excessive activation of serotonin (5-HT) $1 B$ receptors disrupts the formation of sensory maps in monoamine oxidase a and 5-ht transporter knock-out mice. J Neurosci, 2001. 21(3): p. 884-96.

70. Rebsam, A., I. Seif, and P. Gaspar, Refinement of thalamocortical arbors and emergence of barrel domains in the primary somatosensory cortex: a study of normal and monoamine oxidase a knock-out mice. J Neurosci, 2002. 22(19): p. 8541-52.

71. Cases, O., T. Vitalis, I. Seif, E. De Maeyer, C. Sotelo, and P. Gaspar, Lack of barrels in the somatosensory cortex of monoamine oxidase A-deficient mice: role of a serotonin excess during the critical period. Neuron, 1996. 16(2): p. 297-307.

72. Alvarez, C., et al., Effects of genetic depletion of monoamines on somatosensory cortical development. Neuroscience, 2002. 115(3): p. 753-64.

73. Nagatsua, T. and M. Sawadab, L-dopa therapy for Parkinson's disease: past, present, and future. Parkinsonism Relat Disord, 2009. 15 Suppl 1: p. S3-8.

74. Rogers, D.P. and C.A. Goldsmith, Treatment of schizophrenia in the 21st Century: beyond the neurotransmitter hypothesis. Expert Rev Neurother, 2009. 9(1): p. 47-54.

75. Andretic, R., Y.C. Kim, F.S. Jones, K.A. Han, and R.J. Greenspan, Drosophila D1 dopamine receptor mediates caffeine-induced arousal. Proc Natl Acad Sci U S A, 2008. 105(51): p. 20392-7.

76. Pendleton, R.G., F. Parvez, M. Sayed, and R. Hillman, Effects of pharmacological agents upon a transgenic model of Parkinson's disease in Drosophila melanogaster. J Pharmacol Exp Ther, 2002. 300(1): p. 91-6.

77. Haywood, A.F. and B.E. Staveley, Parkin counteracts symptoms in a Drosophila model of Parkinson's disease. BMC Neurosci, 2004. 5: p. 14.

78. Park, J., S.Y. Kim, G.H. Cha, S.B. Lee, S. Kim, and J. Chung, Drosophila DJ-1 mutants show oxidative stress-sensitive locomotive dysfunction. Gene, 2005.

361: p. 133-9.

79. Makos, M.A., Y.C. Kim, K.A. Han, M.L. Heien, and A.G. Ewing, In Vivo Electrochemical Measurements of Exogenously Applied Dopamine in Drosophila melanogaster. Anal Chem, 2009. 